\title{
A PARETO-FRONTIER ANALYSIS OF PERFORMANCE TRENDS FOR SMALL REGIONAL COVERAGE LEO CONSTELLATION SYSTEMS
}

\author{
A Thesis \\ presented to \\ the Faculty of California Polytechnic State University, \\ San Luis Obispo \\ Department of Aerospace Engineering
}

\author{
In Partial Fulfillment \\ of the Requirements for the Degree \\ Master of Science in Aerospace Engineering \\ by \\ Christopher Alan Hinds
}

December 2014 
(C) 2014

Christopher Alan Hinds ALL RIGHTS RESERVED 


\section{COMMITTEE MEMBERSHIP}

TITLE:

AUTHOR:

DATE SUBMITTED:

COMMITTEE CHAIR:

COMMITTEE MEMBER:

COMMITTEE MEMBER:

COMMITTEE MEMBER:
A Pareto-Frontier Analysis of Performance Trends for Small Regional Coverage LEO Constellation Systems

Christopher Alan Hinds

December 2014

Kira J. Abercromby, $\mathrm{PhD}$

Associate Professor of Aerospace Engineering

Eric A. Mehiel, PhD

Department Chair Associate Professor of Aerospace

Engineering

Colleen M. Kirk, PhD

Professor of Mathematics

Jordi Puig-Suari, $\mathrm{PhD}$

Professor of Aerospace Engineering 


\begin{abstract}
A Pareto-Frontier Analysis of Performance Trends for Small Regional Coverage LEO Constellation Systems

Christopher Alan Hinds
\end{abstract}

As satellites become smaller, cheaper, and quicker to manufacture, constellation systems will be an increasingly attractive means of meeting mission objectives. Optimizing satellite constellation geometries is therefore a topic of considerable interest. As constellation systems become more achievable, providing coverage to specific regions of the Earth will become more common place. Small countries or companies that are currently unable to afford large and expensive constellation systems will now, or in the near future, be able to afford their own constellation systems to meet their individual requirements for small coverage regions.

The focus of this thesis was to optimize constellation geometries for small coverage regions with the constellation design limited between 1-6 satellites in a Walker-delta configuration, at an altitude of $200-1500 \mathrm{~km}$, and to provide remote sensing coverage with a minimum ground elevation angle of 60 degrees. Few Pareto-frontiers have been developed and analyzed to show the tradeoffs among various performance metrics, especially for this type of constellation system. The performance metrics focus on geometric coverage and include revisit time, daily visibility time, constellation altitude, ground elevation angle, and the number of satellites. The objective space containing these performance metrics were characterized for 5 different regions at latitudes of $0,22.5,45,67.5$, and 90 degrees. In addition, the effect of minimum ground elevation angle was studied on the achievable performance of this type of constellation system. Finally, the traditional Walker-delta pattern constraint was relaxed to allow for asymmetrical designs. These designs were compared to see how the Walker-delta pattern performs compared to a more relaxed design space. 
The goal of this thesis was to provide both a framework as well as obtain and analyze Pareto-frontiers for constellation performance relating to small regional coverage LEO constellation systems. This work provided an in-depth analysis of the trends in both the design and objective space of the obtained Pareto-frontiers. A variation on the $\mathrm{NSSGA-II}$ algorithm was utilized along with a MATLAB/STK interface to produce these Pareto-frontiers. The $\varepsilon$ NSGA-II algorithm is an evolutionary algorithm that was developed by Kalyanmoy Deb to solve complex multi-objective optimization problems.

The algorithm used in this study proved to be very efficient at obtaining various Paretofrontiers. This study was also successful in characterizing the design and solution space surrounding small LEO remote sensing constellation systems providing small regional coverage. 


\section{ACKNOWLEDGMENTS}

This thesis would not have been possible without the help and support of many people.

First, I would like to thank each member of my thesis committee, Dr. Eric Mehiel, Dr. Jordi Puig-Suari, and Dr. Colleen Kirk. I have learned a great deal from each of these professors during my educational career here at Cal Poly. I am very appreciative of their guidance and input in helping to develop this thesis.

I would also like to thank the people at Analytical Graphics Inc. for providing me with licensing for STK in addition to being extremely helpful whenever I had any questions relating to their software.

A huge thank you goes to my fellow graduate colleagues and friends, Eli Gurnee and Chris Satterwhite. I was always able to rely on them for help and guidance throughout my graduate career here at Cal Poly. In addition, their friendship and presence outside of school related work made it possible to make it through Grad school.

This work would not have been possible without my parents, Jeff and Cathy. Their encouragement, love, and support helped every step of the way and I am forever grateful for the sacrifices they have made to help me along this journey. I am also extremely grateful to have such an incredible sister, Amy, who has provided me with endless inspiration and encouragement along the way. I would also like to thank my wonderful girlfriend, Olivia, for providing love and support throughout my journey here at Cal Poly. To all of the amazing friends I have made here at Cal Poly, I am eternally grateful for the times we have spent together and I hope they continue well into the future.

Finally, I would like to give a huge thank you to my thesis advisor, Dr. Kira Abercromby. I have gained an incredible wealth of knowledge from Dr. A, throughout numerous classes and 
office hours. Her enthusiasm and knowledge of orbital mechanics has inspired me to pursue this thesis as well as hopefully a career in this field. I cannot express how grateful I am to have learned so much from such a wonderful professor in the Aerospace Department here at Cal Poly. Her advice and help throughout this entire study has made this thesis possible and I am forever grateful.

Good luck and Godspeed. 


\section{TABLE OF CONTENTS}

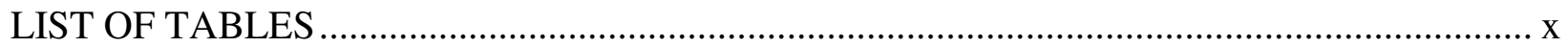

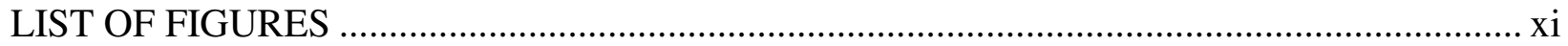

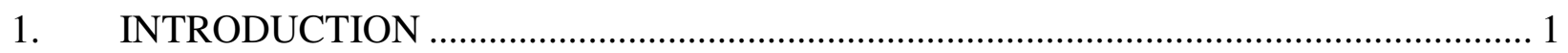

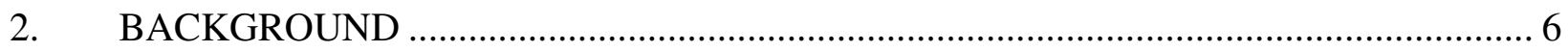

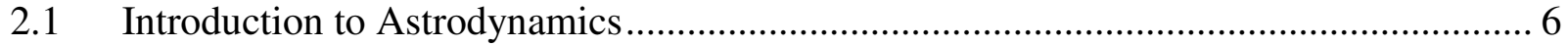

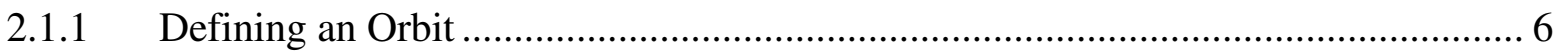

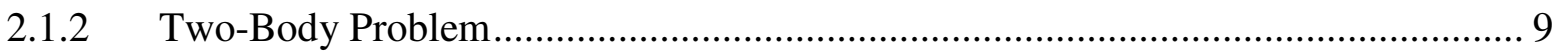

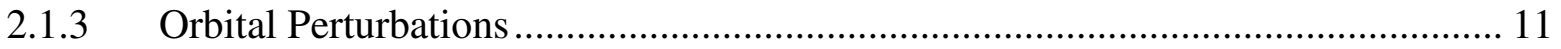

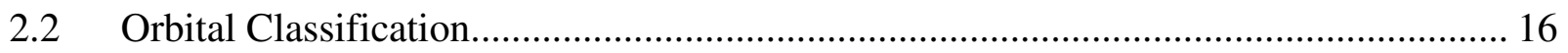

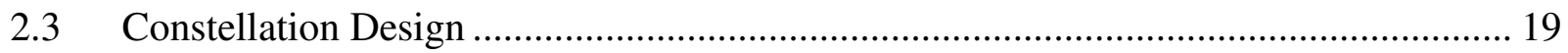

2.3.1 Traditional Constellation Patterns.......................................................................... 19

2.3.2 Constellation Mission Classification ……………............................................ 22

2.3.3 Constellation Performance Metrics................................................................... 24

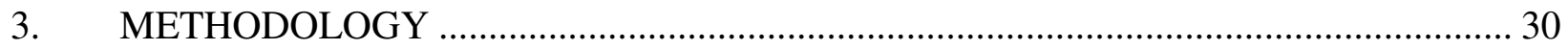

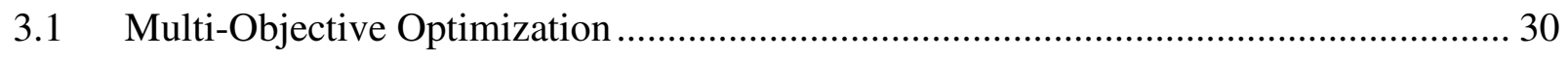

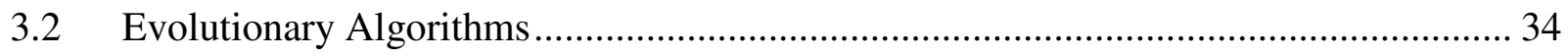

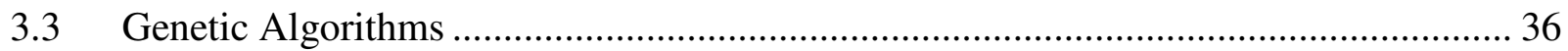

3.4 Multi-Objective Genetic Algorithms ...................................................................... 47

3.4.1 Variations on the Multi-Objective Genetic Algorithm ......................................... 47

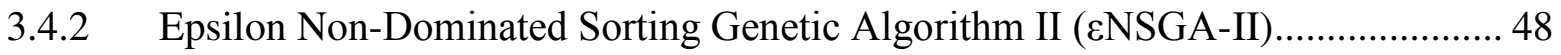

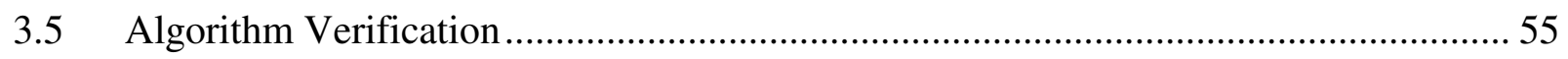

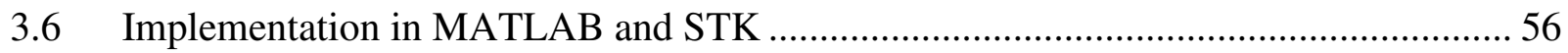

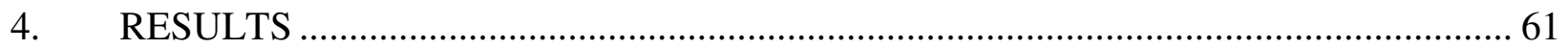

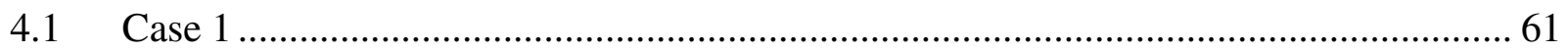

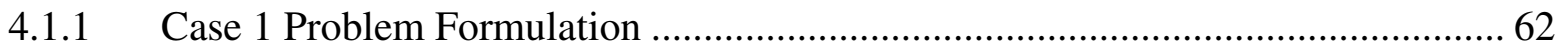

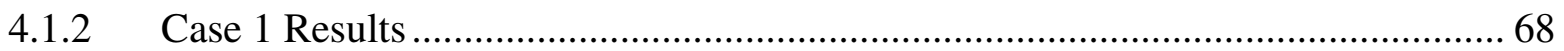

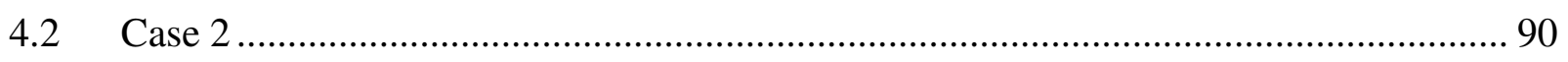

4.2.1 Case 2a Problem Formulation.............................................................................. 91

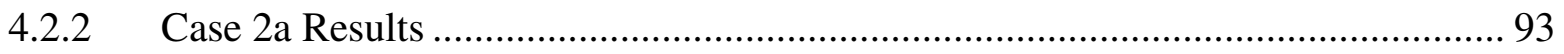


4.2.3 Case 2b Problem Formulation ...................................................................... 96

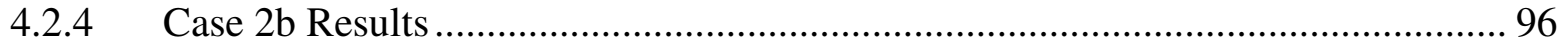

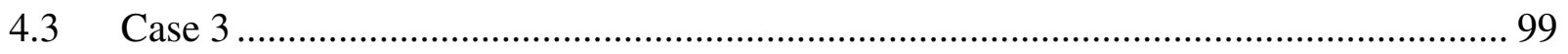

4.3.1 Case 3 Problem Formulation ...................................................................... 99

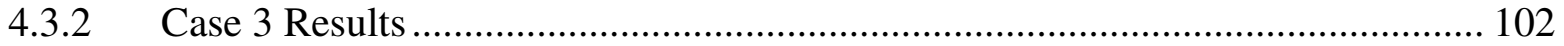

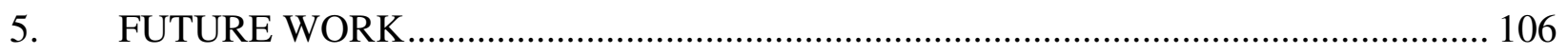

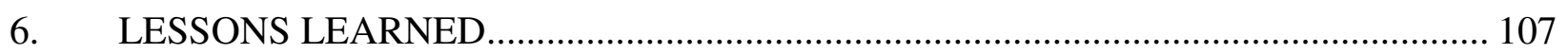

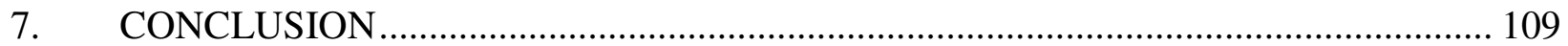

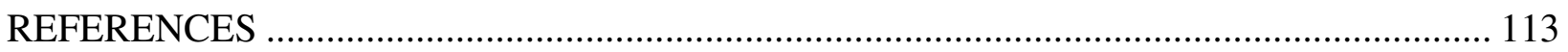




\section{LIST OF TABLES}

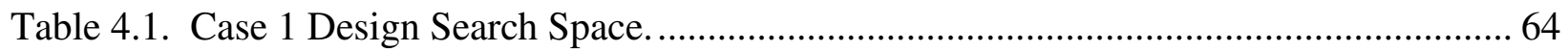

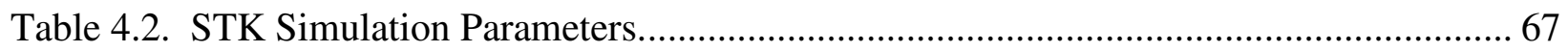

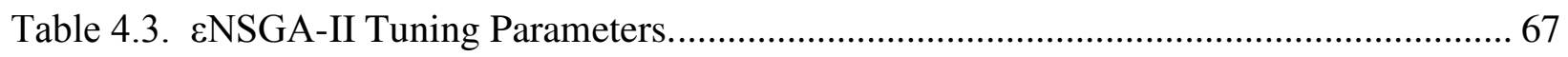

Table 4.4. Case 1 Design Objectives and Corresponding Epsilon Grid Values......................... 68

Table 4.5. Case 2 Design Search Space......................................................................... 92

Table 4.6. Case 2a Design Objectives and Corresponding Epsilon Grid Values..................... 92

Table 4.7. Case 2b Design Objectives and Corresponding Epsilon Grid Values..................... 96

Table 4.8. Case 3 Design Objectives and Corresponding Epsilon Grid Values...................... 100

Table 4.9. Case 3 Design Search Space for the Walker-Delta Pattern. ................................. 100

Table 4.10. Case 3 Design Search Space for the Relaxed Constellation Design...................... 102 


\section{LIST OF FIGURES}

Figure 2.1. Classical Orbital Elements Diagram .............................................................. 7

Figure 2.2. Geometry for Two-Body Problem with Inertial Reference Frame ........................ 10

Figure 2.3. Earth's Gravitational Potential Field............................................................... 14

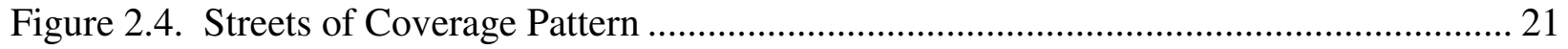

Figure 2.5. Earth Coverage Geometry ...................................................................... 25

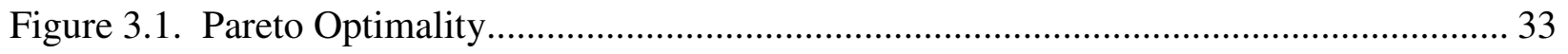

Figure 3.2. Basic Genetic Algorithm Flow Chart ........................................................... 36

Figure 3.3. Basic Genetic Algorithm Pseudo Code ....................................................... 37

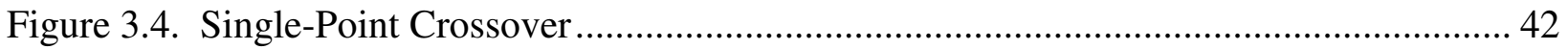

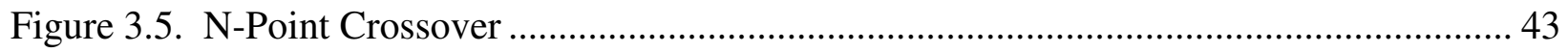

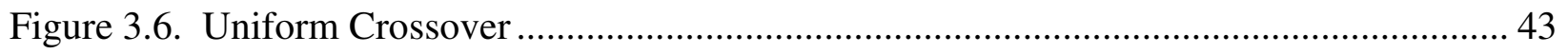

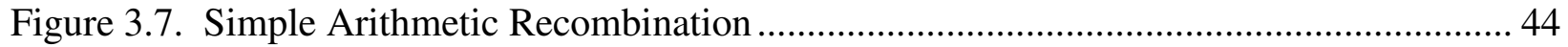

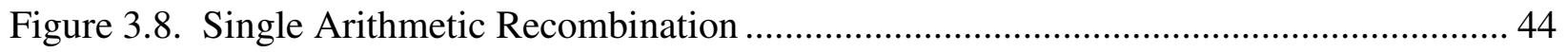

Figure 3.9. Whole Arithmetic Recombination....................................................................... 44

Figure 3.10. Potential Area of Children from Two Parents ................................................ 45 
Figure 3.11. Bit Flip Mutation for Binary Representation 46

Figure 3.12. Epsilon-dominance 48

Figure 3.13. Diagram of the eNSGA-II algorithm as shown in [32] 49

Figure 3.14. Crowding Distance Cuboid 52

Figure 3.15. ZDT4 Test Function Verification. 56

Figure 3.16. MATLAB/STK Interface Schematic. 58

Figure 3.17. STK Walker Constellation 3D Visualization 59

Figure 3.18. STK Walker Constellation 2D Ground Track Visualization 60

Figure 4.1. Case 1 Chromosome Structure and Resulting Makeup of Constellation COEs 64

Figure 4.2. Number of Spacecraft vs. Constellation Altitude vs. MDVT vs. MRT for 0 Degree Latitude Target. 70

Figure 4.3. Number of Spacecraft vs. Constellation Altitude vs. MDVT vs. MRT for 22.5 Degree Latitude Target 70

Figure 4.4. Number of Spacecraft vs. Constellation Altitude vs. MDVT vs. MRT for 45 Degree Latitude Target 71

Figure 4.5. Number of Spacecraft vs. Constellation Altitude vs. MDVT vs. MRT for 67.5 Degree Latitude Target 71

Figure 4.6. Number of Spacecraft vs. Constellation Altitude vs. MDVT vs. MRT for 90 Degree Latitude Target 72 
Figure 4.7. Number of Spacecraft vs. MDVT vs. MRT for 45 Degree Latitude Target, with the Number of Planes Shown in Color

Figure 4.8. Number of Spacecraft vs. MDVT vs. Constellation Altitude for 0 Degree Latitude

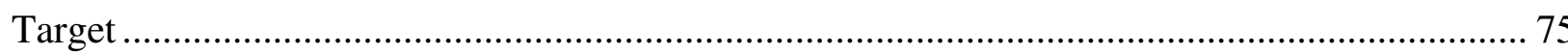

Figure 4.9. Number of Spacecraft vs. MDVT vs. Constellation Altitude for 22.5 Degree

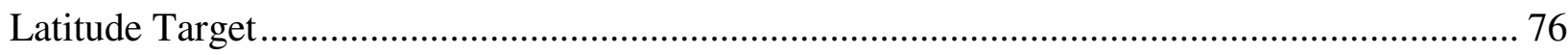

Figure 4.10. Number of Spacecraft vs. MDVT vs. Constellation Altitude for 45 Degree

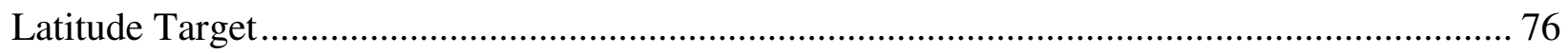

Figure 4.11. Number of Spacecraft vs. MDVT vs. Constellation Altitude for 67.5 Degree

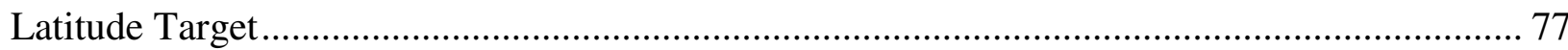

Figure 4.12. Number of Spacecraft vs. MDVT vs. Constellation Altitude for 90 Degree

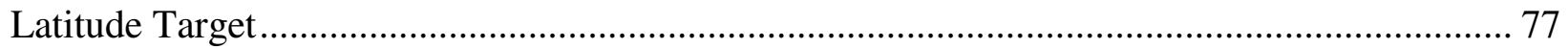

Figure 4.13. Number of Spacecraft vs. MDVT vs. Constellation Altitude for 0 Degree Latitude Target, with the Constellation Inclination in Color 79

Figure 4.14. Number of Spacecraft vs. MDVT vs. Constellation Altitude for 22.5 Degree Latitude Target, with the Constellation Inclination in Color 80

Figure 4.15. Number of Spacecraft vs. MDVT vs. Constellation Altitude for 45 Degree Latitude Target, with the Constellation Inclination in Color. 80

Figure 4.16. Number of Spacecraft vs. MDVT vs. Constellation Altitude for 67.5 Degree Latitude Target, with the Constellation Inclination in Color. 81

Figure 4.17. Number of Spacecraft vs. MDVT vs. Constellation Altitude for 90 Degree Latitude Target, with the Constellation Inclination in Color 81 
Figure 4.18. Number of Spacecraft vs. Constellation Altitude vs. MRT for 0 Degree Latitude Target

Figure 4.19. Number of Spacecraft vs. Constellation Altitude vs. MRT for 22.5 Degree Latitude Target.

Figure 4.20. Number of Spacecraft vs. Constellation Altitude vs. MRT for 45 Degree Latitude Target.

Figure 4.21. Number of Spacecraft vs. Constellation Altitude vs. MRT for 67.5 Degree Latitude Target.

Figure 4.22. Number of Spacecraft vs. Constellation Altitude vs. MRT for 90 Degree Latitude Target.

Figure 4.23. Number of Spacecraft vs. MRT for 0 Degree Latitude Target, with the Number of Planes Shown in Color 86

Figure 4.24. Number of Spacecraft vs. MRT for 22.5 Degree Latitude Target, with the Number of Planes Shown in Color

Figure 4.25. Number of Spacecraft vs. MRT for 45 Degree Latitude Target, with the Number of Planes Shown in Color

Figure 4.26. Number of Spacecraft vs. MRT for 67.5 Degree Latitude Target, with the Number of Planes Shown in Color

Figure 4.27. Number of Spacecraft vs. MRT for 90 Degree Latitude Target, with the Number of Planes Shown in Color

Figure 4.28. Case 2 Chromosome Structure

Figure 4.29. Minimum Ground Elevation Angle vs. Constellation Altitude vs. Mean DVT for Target Region at 45 Degrees Latitude 
Figure 4.30. Minimum Ground Elevation Angle vs. Constellation Altitude vs. Mean DVT for Target Region at 45 Degrees Latitude

Figure 4.31. Minimum Ground Elevation Angle vs. Constellation Altitude vs. Mean DVT for Target Region at 45 Degrees Latitude

Figure 4.32. Minimum Ground Elevation Angle vs. Constellation Altitude vs. Mean Revisit Time for Target Region at 45 Degrees Latitude.

Figure 4.33. Minimum Ground Elevation Angle vs. Constellation Altitude vs. Mean Revisit Time for Target Region at 45 Degrees Latitude

Figure 4.34. Case 3 Chromosome Structure and Resulting Makeup of Constellation COEs for the Walker-Delta Pattern..... 100

Figure 4.35. Case 3 Chromosome Structure and Resulting Makeup of Constellation COEs for the Relaxed Constellation Design 101

Figure 4.36. Constellation Altitude vs. MRT vs. MDVT for the Walker-Delta Pattern 103

Figure 4.37. Constellation Altitude vs. MRT vs. MDVT for the Relaxed Constellation Design 104 


\section{INTRODUCTION}

Space based systems provide an extremely unique opportunity for data collection and transmission, that cannot currently be accomplished without the efficiency that satellites provide. Spacecraft have the ability to view large amount of the Earth's surface at a given time, and thus fulfill certain niches in our modern world including communication, navigation, and remote sensing. Many mission requirements can be met by using multiple spacecraft working together in concert, known as satellite constellations. Constellation systems allow either continuous coverage over a given region or discontinuous coverage that is improved over what a single satellite may achieve. Satellite constellations provide an attractive means of accomplishing many different types of mission goals. The GPS constellation system for example has revolutionized the field of navigation by providing a variety of users with the ability to efficiently and accurately locate their exact position on the Earth. Constellation systems such as RapidEye allow users in agriculture, environmental studies, emergency response, infrastructure, and other fields to obtain geospatial data which provide a basis for studying the environment. Remote sensing constellations such as RapidEye have made it possible to study our planet on a very large scale, from which we have obtained a vast amount of knowledge relating to the sustainability of our environment. The Iridium and Globalstar constellations have allowed users to communicate via satellite phones in regions where traditional communication methods would not allow. These specific niches that constellation systems fulfill will only continue to grow as satellites become smaller, cheaper, and quicker to manufacture.

As constellation systems become more achievable, providing coverage to specific regions of the Earth will become more common place. Small countries or companies that are currently unable to afford large and expensive constellation systems will now, or in the near future, be able 
to afford their own constellation systems to meet their individual requirements. Small satellite constellation systems will provide a cheap and viable solution to meet a variety of goals including regional remote sensing, communications, and navigation. As these constellation systems are developed for small regions of the Earth, it is important to understand the tradeoffs in the cost and performance of these systems. Providing coverage to small regions of the Earth will become increasingly prevalent for small imaging constellation systems. Very few studies exist which analyze the trends in performance tradeoffs for constellation systems, especially for regional remote sensing systems constellations.

The purpose of this study is to provide mission designers with not only the methodology to analyze these trends for their specific system, but also present and characterize the design space surrounding these types of systems. The main goal of this work is to characterize the design space surrounding small regional LEO remote sensing constellation designs. A secondary goal is to see how the latitude of a small region of interest affects the achievable performance and geometrical design of a small regional LEO remote sensing constellation system. Obtaining Pareto-frontiers that show trends in conflicting performance metrics such as the number of satellites in the constellation, altitude/resolution, daily visibility time, revisit time, and minimum elevation angle can be incredibly useful to mission designers. Comparing these metrics for small regions of interest at varying latitudes will show interesting trends, in addition to providing mission designers with an indication of the performance that is achievable at specific latitudes. In this study, an evolutionary algorithm is implemented in MATLAB and a connection to STK is established to compute constellation performance. MATLAB is a numerical computing programming language, developed by Analytical Graphics Inc. (AGI), which allows for easy implementation of algorithms, mostly intended for engineering and science users. STK is a 
physics-based software that was created by Analytical Graphics, Inc. to compute spatial relationships, or accesses, between assets through numerical simulations. STK was originally developed to provide access information for Earth-orbiting spacecraft, but has since expanded to many other applications including the analysis of ground, sea, and air assets.

In this study a variation on an evolutionary algorithm called the ENSGA-II, is shown to be very effective at obtaining Pareto-frontiers for these conflicting performance metrics. The ¿NSGA-II is an evolutionary algorithm that was developed by Kalyanmoy Deb to solve complex multi-objective optimization problems. Many previous studies have explored satellite constellation design using evolutionary algorithms. In the later part of the 1990s, Frayssinhes et al. investigated the use of genetic algorithms in developing new satellite constellation geometries through several studies [25, 26, 27]. Frayssinhes et al. found that gains in constellation performance beyond traditional designs could be found using the approach of genetic algorithms. Smith utilized a parallel genetic algorithm to design and optimize a variation on the Ellipso constellation [43]. Asvial, Tafazolli, and Evans have produced several studies appling genetic algorithms to optimize non-GEO satellite constellations as well as hybrid constellation designs $[5,6]$. In a Master's Thesis, Pegher and Parish utilize a genetic algorithm to optimize coverage and revisit time in sparse military satellite constellations [38]. In a Master's Thesis, Bruccoleri utilized a genetic algorithm to optimize specific flower constellation designs [10]. In addition to constellation design, genetic algorithms have been extremely effective at optimizing the orbit of a single satellite for various missions and constraints [1, 2, 47].

Several studies have used Pareto-based analysis to characterize the objective space surrounding constellation design. Mason et al. show how Pareto-based genetic algorithms along with STK can be used to analyze tradeoffs in constellation performance for continuous global 
coverage [36]. In several studies, De Weck shows how Pareto-frontiers can be used to visualize how design choices can affect the final design, especially relating to the life cycle cost of constellation development [13,14]. Mao developed an improved NSGA-II algorithm to obtain Pareto-frontiers to help solve constellation design problems [35]. The focus of Mao's work was on determining the best operators and structure of the genetic algorithm when applied to constellation design problems. Wang utilized the NSGA-II algorithm along with STK to design regional coverage reconnaissance satellite constellations [48]. In multiple studies, Ferringer has utilized Pareto-frontier analysis through the use of genetic algorithms to try and analyze the objective space surrounding several specific constellation design problems. In Ferringer's Master's Thesis he obtained a general framework, along with Pareto-frontiers, for reconfiguring a constellation for optimal performance after suffering a spacecraft loss [20]. In addition, Ferringer et al. have analyzed conflicting performance trends between revisit time and spatial resolution for sparse-coverage constellations [23]. Ferringer et al. have also attempted to characterize the design space surrounding global Walker constellations using Pareto-frontier analysis [22]. As seen, a decent amount of past work has gone into Pareto-frontier analysis of constellation design. Although a lot of work has been done in this field, there is generally a lack of constellation performance tradeoff analysis due to the vast field that is constellation design.

The rest of this paper is structured as follows. Chapter 2 provides a background from which the rest of the study is built on. An introduction to astrodynamics, along with constellation design methodologies and performance metrics will be presented. Chapter 3 provides an introduction as well as a detailed description of the tools used to obtain the results of this study. Multi-objective optimization techniques will be presented, along with a detailed discussion of the algorithm utilized in this study. In addition, an introduction to the software 
used in this study, specifically MATLAB and STK, will be presented. Chapter 4 contains a detailed analysis and discussion of the results of this study. Here, the results are presented and several conclusions are made. Chapter 5 provides a reflection on the results of this study, along with suggestions made for future constellation design based on these results. Suggestions for future work brought about by this study are also presented. 


\section{BACKGROUND}

This chapter presents a brief introduction to orbital mechanics, necessary to understand the complex interaction between the orbits of multiple satellites in a constellation. In addition, several fundamental assumptions will be presented which will allow the derivation of the Equations of Motion (EOM) of a satellite. Finally, a discussion of specialized orbits, constellation design methodologies, and constellation performance metrics will be presented.

\subsection{Introduction to Astrodynamics}

Celestial mechanics is a field within astronomy that deals with the motion of celestial objects, and has provided the foundation of modern day orbital mechanics. Orbital mechanics, or astrodynamics, is the study of dynamics and orbits concerning artificial satellites. Spacecraft are subject to an incredibly complex set of natural forces, along with artificial forces, which must be fully understood in order to accurately model and design space missions. This section presents the basic formulation of satellite dynamics.

\subsubsection{Defining an Orbit}

The six classical orbital elements (COE's) are the most common way of defining the orbit of an object in space and time. With the following six parameters, an objects location and trajectory in space may be exactly determined. Figure 2.1 depicts four of the six COE's, along with several other key terms. 


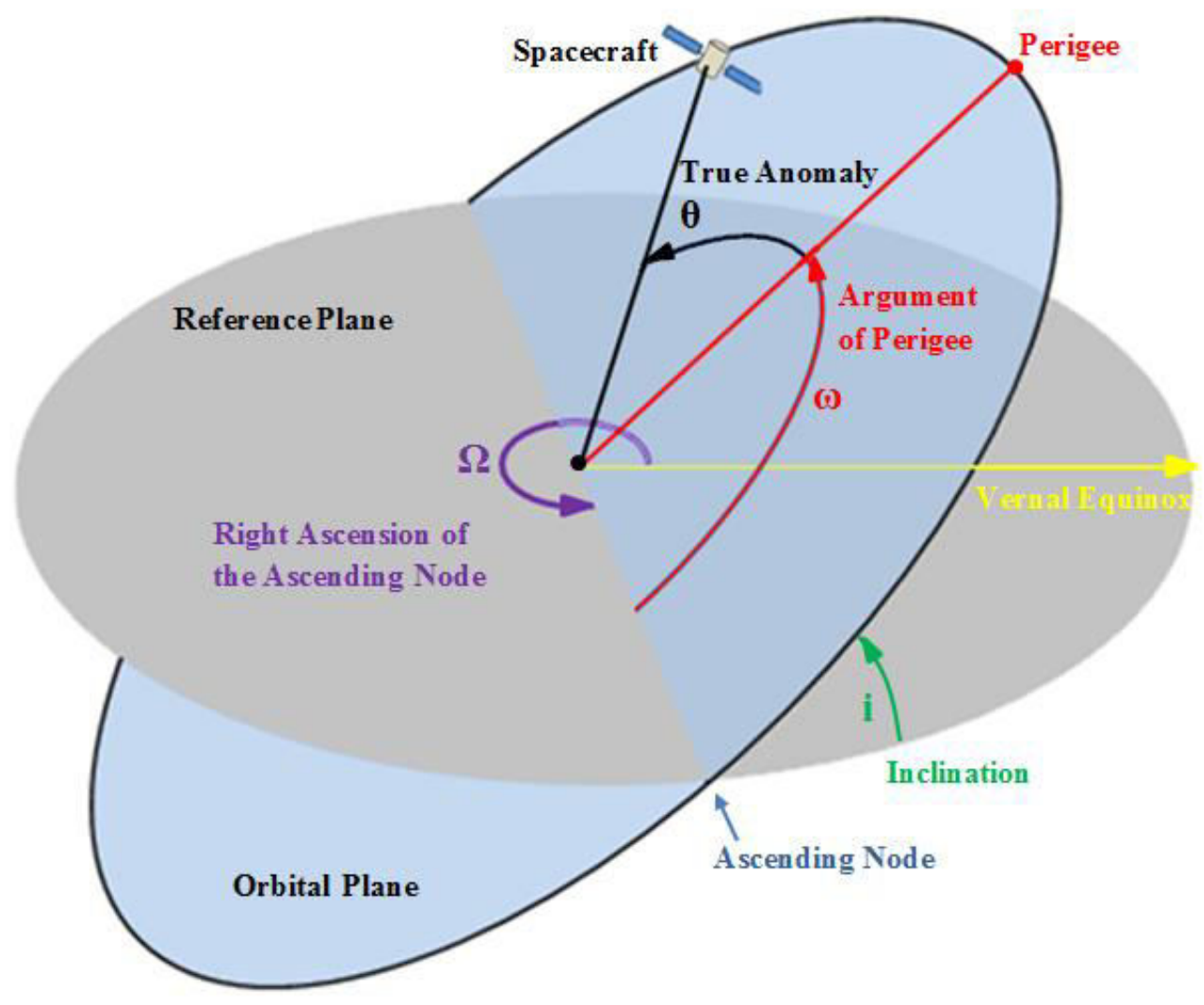

Figure 2.1. Classical Orbital Elements Diagram. This diagram was created based on [44], and depicts several of the classical orbital elements used to define an object in orbit. The orbital elements shown in this diagram include inclination, argument of perigee, right ascension of the ascending node, and the true anomaly.

- Semimajor Axis (a): The semimajor axis describes the size of the orbit, and is determined by half the distance along the axis between the periapsis and the apoapsis. The periapsis is the point in the orbit closest to the center of the body about which the satellite is orbiting; the apoapsis is the point farthest from the center of the body. For circular orbits the semimajor axis is the distance between the centers of the two bodies. The semimajor axis is usually expressed in units of kilometers.

- Eccentricity (e): Eccentricity describes the shape of the orbit. An eccentricity of 0 is a circular orbit, an eccentricity between 0 and 1 is an ellipse, an eccentricity of 1 is a parabolic orbit, and an eccentricity greater than 1 is a hyperbolic orbit. The eccentricity 
is mathematically given by the ratio of the distance between two foci and the semimajor axis.

- Inclination (i): The inclination describes the angular tilt of the orbital plane with respect to a reference plane. It is the angle between the plane of the coordinate system and the orbital plane, between 0 and 180 degrees. For an Earth centered inertial (ECI) system, a satellite with an inclination of 0 would be orbiting in the same plane as the equator, whereas an inclination of 90 would be passing directly over the poles. It is important to note that an inclination of $0^{\circ} \leq i<90^{\circ}$ is said to be prograde, with the satellite orbiting in the same direction of the rotation of the primary body; an inclination of $90^{\circ}<i \leq$ $180^{\circ}$ is retrograde, with the satellite orbiting in the opposite direction of the rotation of the primary body.

- Argument of Perigee ( $\omega)$ : The argument of perigee describes the orientation of the ellipse with respect to the orbital plane. It is defined as the angle between 0 and 360 degrees between the ascending node (the point at which the satellite passes upwards from the southern hemisphere to the northern hemisphere) and the periapsis in the orbital plane.

- Right Ascension of the Ascending Node ( $\Omega$ ): The RAAN is used to describe the point in the orbit where the satellite passes upwards through the reference plane. It therefore describes the angular orientation where the reference plane and the orbital plane intersect. It is measured as the angle between 0 and 360 degrees between the vernal equinox (the vector between the Earth and Sun on the first day of spring) and the ascending node.

- True Anomaly (v): Finally, the true anomaly describes the satellites location in the orbit. It is defined as the angle between 0 and 360 degrees within the orbital plane between the 
periapsis point and the satellite's position vector (from the center of the primary body to the satellite), measured in the direction of the satellite's motion.

\subsubsection{Two-Body Problem}

The two-body problem in classical mechanics is determining the motion of two bodies that interact only with each other. Several assumptions are required to reduce this problem to its simplest form; the two objects are modeled strictly as point masses and there are no external or internal forces besides gravitational forces which act along a line between the two point masses. A satellite orbiting the Earth or an electron orbiting an atomic nucleus are examples of the twobody problem. Here, the EOM for a two-body system will be presented before more complicated models are introduced.

Newton's second law of motion can be mathematically represented by,

$$
\sum \vec{F}=m \ddot{\vec{r}}
$$

where the force, $\vec{F}$, acting on a body is equal to the mass, m, multiplied by the acceleration, $\ddot{\vec{r}}$, of the body.

From the laws of motion Newton developed the Universal Law of Gravitation, which states that any two masses will attract each other with a force that is proportional to the product between their masses and inversely proportional to the square of the distance between them. This can be represented mathematically by,

$$
\overrightarrow{F_{g}}=-\frac{G M m}{r^{2}}\left(\frac{\vec{r}}{r}\right)
$$


where the gravitational force, $\mathrm{F}_{\mathrm{g}}$, is equal to the product of the two masses $\mathrm{M}$ and $\mathrm{m}$, the universal gravitational constant which is equal to $6.67384 \times 10^{-11} \frac{\mathrm{m}^{3}}{\mathrm{~km} \mathrm{~s}^{2}}$, divided by the distance between the two masses squared, $r^{2}$. The universal law of gravitation applies to any two mass objects. Newton's universal law of gravitation along with his three laws of motion enabled future scientists and astronomers to model the dynamic interaction among celestial bodies and satellite motion. These fundamental equations are used as the building blocks for complex satellite motion.

The geometry for the two-body system is shown in Figure 2.2 for the Earth-satellite system along with the reference frames.

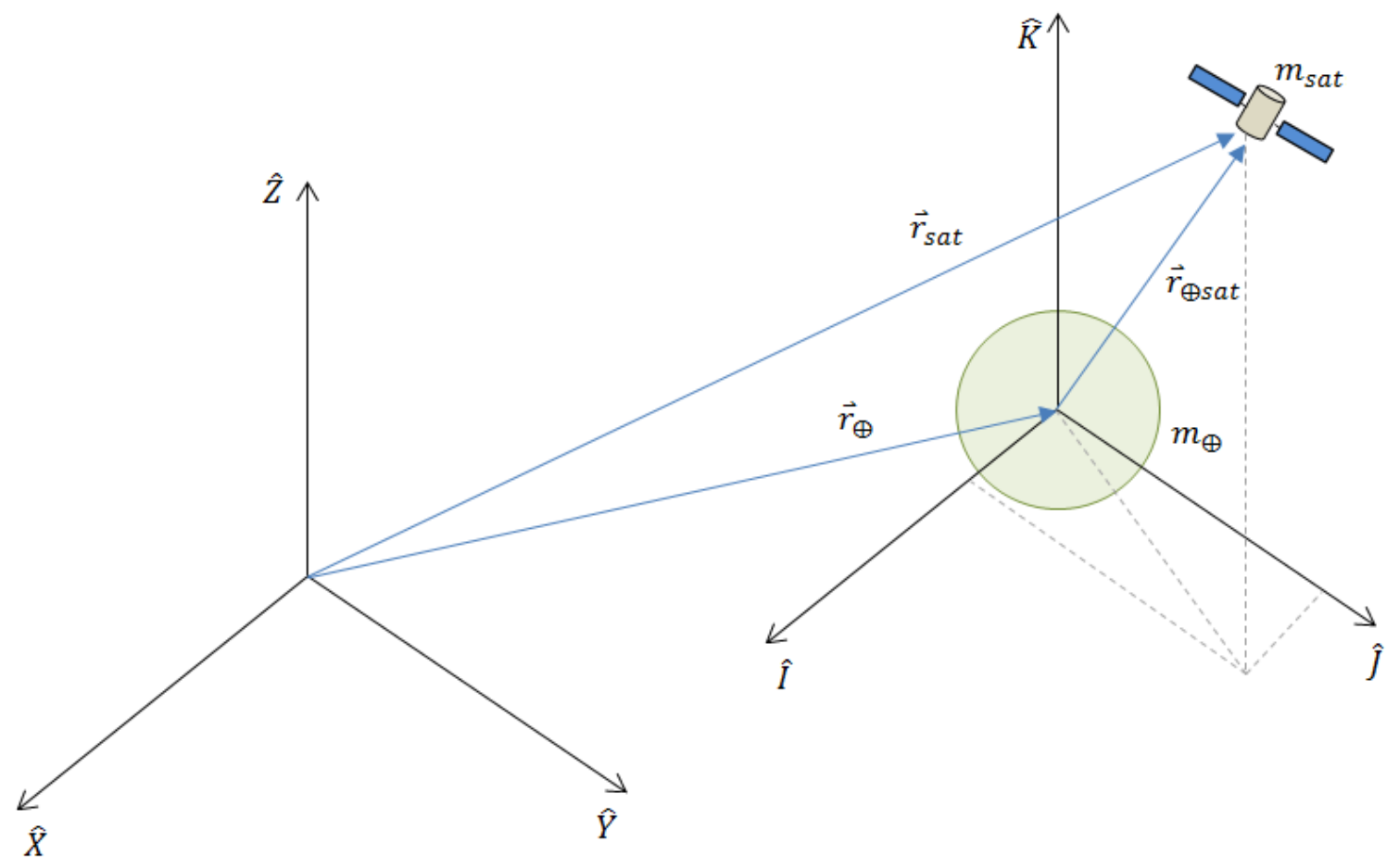

Figure 2.2. Geometry for Two-Body Problem with Inertial Reference Frame. This diagram was created based off Vallado [45], and depicts the various vectors and reference frames used in the derivation of the two-body solution. $X Y Z$ is an inertial reference frame, where IJK is a reference frame removed from $X Y Z$ and does not rotate or accelerate with respect to $X Y Z$. 
The solution to the two-body problem is given as,

$$
\ddot{\vec{r}}_{\oplus s a t}=-\frac{\mu}{r_{\oplus s a t}{ }^{2}}\left(\frac{\vec{r}_{\oplus s a t}}{r_{\oplus s a t}}\right)
$$

where the standard gravitational parameter, $\mu$, is the product of the gravitational constant, $\mathrm{G}$, and the mass of the primary body; in the case of the Earth, $\mu=398,600.4418 \frac{\mathrm{km}^{3}}{\mathrm{~s}^{2}}$.

Equation 2.3 provides a formulation for a numerical solution to the two-body problem, where the position and velocity as a function of time for a satellite may be solved using integration. It must be remembered however, this equation represents a solution for pure Keplerian motion. In reality there are many other forces acting on a satellite which act to perturb the orbit. The four main perturbing forces include atmospheric drag, solar radiation pressure (SRP), third body effects, and Earth oblateness.

\subsubsection{Orbital Perturbations}

In order to more accurately model the orbital motion of a satellite, the assumptions made in the two-body problem will be relaxed and additional forces will be added to produce a more complex, yet accurate model. The four main perturbing forces as previously stated are atmospheric drag, solar radiation pressure (SRP), n-body effects, and the non-spherical Earth. Although these are the main perturbing forces, additional forces of much smaller magnitude act on a satellite. These smaller forces include tidal friction, magnetic field interactions, relativistic effects, and artificially produced forces. The only perturbative forces that are utilized in this study are the forces due to the Earth as an oblate spheroid. For this reason a derivation will be

provided only for this perturbative force, with brief explanations of the other main perturbative forces.

\section{Non-Spherical Earth:}


The two-body problem relied on the key assumption that the two masses involved are considered to be point masses. This resulted in a simplified gravitational potential, $\frac{\mu}{r}$, which does not account for the actual shape of the Earth. In reality the Earth is not spherically symmetrical but is bulged at the equator, flattened in the polar regions, and contains large and seemingly random variations in the gravitational potential. The largest perturbative force due to the variation in the gravitational potential is the bulge at the equator, which is known as the $\mathbf{J}_{2}$ perturbation. The oblateness of the Earth causes large changes over time in several orbital elements when compared to pure Keplerian motion. It is therefore often necessary to include the $\mathbf{J}_{2}$ effect in order to get an accurate model. The following derivation from [9] provides a new relationship for the satellite EOM, which includes the secular effects of the Earth's oblateness.

Take the gravitational potential function for a spheroid,

$$
\Phi_{\text {sphere }}=\frac{\mu}{r}
$$

where the gravitational potential function, $\Phi$, is equal to the standard gravitational parameter divided by the distance between the two bodies. The acceleration of the secondary body is then found by taking the gradient of the potential function as follows,

$$
\ddot{\vec{r}}=\vec{\nabla} \Phi=\frac{\delta \Phi}{\delta x} \hat{I}+\frac{\delta \Phi}{\delta y} \hat{Y}+\frac{\delta \Phi}{\delta z} \hat{Z}
$$

where $\nabla$ is the gradient operator. It follows that the gradient of the potential function of a perfect spheroid in the ECI coordinate frame is,

$$
\ddot{\vec{r}}_{\text {sphere }}=\vec{\nabla} \Phi_{\text {sphere }}=-\frac{1}{2} \frac{\mu}{\left(x^{2}+y^{2}+z^{2}\right)^{3 / 2}}[2 x \hat{I}+2 y \hat{\jmath}+2 z \widehat{K}]=-\frac{\mu}{r^{3}} \vec{r} .
$$

Note that this acceleration vector is the same solution found for the two-body problem in Eqn. 2.3. In order to derive the EOM for an asymmetrical body, a different gravitational potential function must be used. One potential function for the Earth given by Vinti [46] that is based solely on the zonal harmonics is, 


$$
\Phi=\frac{\mu}{r}\left[1-\sum_{n=2}^{\infty} J_{n}\left(\frac{r_{e}}{r}\right)^{n} P_{n} \sin (\phi)\right]
$$

where $J_{n}$ are coefficients determined by experimental observation, $r_{e}$ is the equatorial radius of the Earth, $\mathrm{P}_{\mathrm{n}}$ are the Legendre polynomials, and $\phi$ is the geocentric latitude where,

$$
\sin (\phi)=\frac{z}{r}
$$

The first $6 \mathrm{~J}$ coefficients for the zonal harmonics are displayed below as given by Baker [7]:

$$
\begin{aligned}
& J_{2}=(1082.64 \pm 0.03) \times 10^{-6} \\
& J_{3}=(-2.5 \pm 0.1) \times 10^{-6} \\
& J_{4}=(-1.6 \pm 0.5) \times 10^{-6} \\
& J_{5}=(-0.15 \pm 0.1) \times 10^{-6} \\
& J_{6}=(0.57 \pm 0.1) \times 10^{-6} \\
& J_{7}=(-0.44 \pm 0.1) \times 10^{-6}
\end{aligned}
$$

It is seen that the $J_{2}$ coefficient is over 400 times larger than the $J_{3}$ coefficient. The $J_{2}$ coefficient represents the equatorial bulge and is the only term that will be considered for the rest of the derivation due to its significance over the other coefficients. The Legendre polynomial for the second term is given by,

$$
P_{2}[\sin (\phi)]=\frac{1}{2}\left[3 \sin ^{2}(\phi)-1\right] .
$$

Using just the $\mathrm{J}_{2}$ term and substituting the Legendre polynomial into Eqn. 2.7, the first term of the potential function due to the equatorial bulge is,

$$
\phi=\frac{\mu}{r}\left[1-\frac{J_{2}}{2}\left(\frac{r_{e}}{r}\right)^{2}\left(3 \sin ^{2}(\phi)-1\right)\right]
$$

Given that the acceleration is equal to the gradient of the gravitational potential function, and noting that,

$$
r=\sqrt{x^{2}+y^{2}+z^{2}}
$$

along with the relationship given for the geocentric latitude in Eqn. 2.8, we obtain the following, 


$$
\begin{aligned}
& \ddot{x}=\frac{\delta \Phi}{\delta x}=-\frac{\mu x}{r^{3}}\left[1-J_{2} \frac{3}{2}\left(\frac{r_{e}}{r}\right)^{2}\left(5 \frac{z^{2}}{r^{2}}-1\right)\right] \\
& \ddot{y}=\frac{\delta \Phi}{\delta y}=-\frac{\mu y}{r^{3}}\left[1-J_{2} \frac{3}{2}\left(\frac{r_{e}}{r}\right)^{2}\left(5 \frac{z^{2}}{r^{2}}-1\right)\right] \\
& \ddot{z}=\frac{\delta \Phi}{\delta z}=-\frac{\mu z}{r^{3}}\left[1+J_{2} \frac{3}{2}\left(\frac{r_{e}}{r}\right)^{3}\left(3-5 \frac{z^{2}}{r^{2}}\right)\right] .
\end{aligned}
$$

Similar to Similar to Eqn. 2.3, Eqn. 2.12 may be integrated numerically to determine the position and velocity of a satellite given some initial conditions. This will provide a more accurate model of the orbit due to the fact that the secular effects of the $\mathrm{J}_{2}$ term are included in the propagation. The method of adding in acceleration terms to the two-body

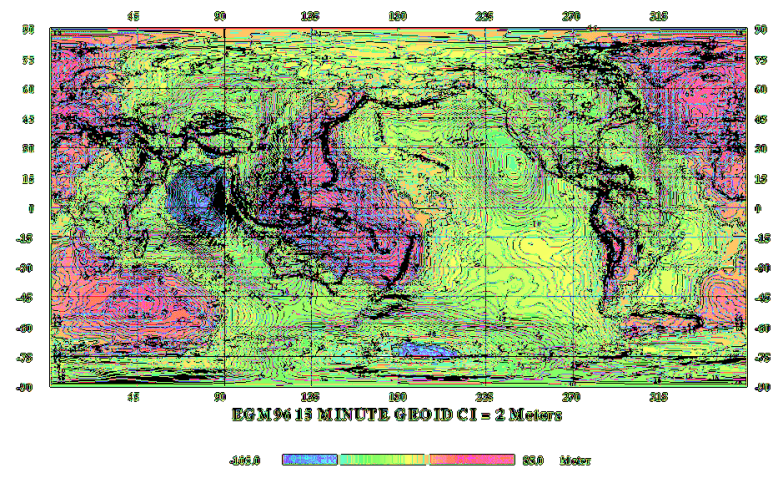

Figure 2.3. Earth's Gravitational Potential Field. This image depicts the anomalies in Earth's gravitational potential as the geoid height in meters. This model was developed by NASA in 1996 and utilizes a spherical harmonic model to order and degree 360. [37]

acceleration is known as Cowells Method and is shown in Eqn. 2.12.

It should be noted that the $\mathbf{J}_{2}-\mathrm{J}_{7}$ equations provide only the zonal harmonics, which depend on the mass distribution that varies only with latitude. Other harmonics include the sectorial harmonics which are dependent only on longitude, and the tesseral harmonics which are dependent on both latitude and longitude. Figure 2.3 shows a representation of the gravitational potential field over the Earth that includes the zonal, sectorial, and tesseral harmonics.

\section{N-Body Effects:}

Another perturbation force that can affect the orbit of a satellite is the influence of gravitational fields other that of the Earth. When modeling the orbit of a satellite that is in Earth orbit but has a relatively large semimajor axis, it is important to take into account the 
gravitational attraction of the moon. For interplanetary trajectories, modeling the gravitational attractions of other planets or the sun is a must. In order to further modify the satellite EOM to account for n-body interactions, you must add both direct and indirect accelerations due to the presence of additional bodies. The direct acceleration is due to the force acting on the satellite by the additional body, and the indirect acceleration is due to the force on the primary body by the additional body.

\section{Atmospheric Drag:}

For satellites orbiting below an altitude of about $1,500 \mathrm{~km}$, atmospheric drag can play an important role in the forces acting on the satellite. Although the atmosphere has a relatively small density, the satellite is traveling at extremely high speeds which can introduce a large drag force. Drag acts in the direction opposite of the velocity vector, thereby slowing the satellite and removing energy from the orbit. For objects traveling in an orbit below $150 \mathrm{~km}$, the lifetime is on the order of several days and the object will reenter the atmosphere quickly. Developing accurate drag models is extremely difficult due to the fluctuations in atmospheric density along with uncertainties in the frontal area of the orbiting object. Several models for the atmospheric density which vary by time and altitude exist. The most basic model is the exponential model which varies in altitude, with more complicated models based on time in addition to altitude being the Russian GOST model, US Naval Research Laboratory Mass Spectrometer and Incoherent Scatter Radar 2000 model (NRLMSISE-00), and the Jacchia Reference model.

\section{Solar Radiation Pressure:}

One of the smaller perturbative forces is a pressure force due to solar radiation. SRP is a non-conservative force that varies with the sun angle and is larger at higher altitudes. SRP can be extremely difficult to model due to the varying cross sectional area of the satellite, modeling 
satellite reflectivity, solar flux variations, and passages into the umbra and penumbra of the Earth.

\subsection{Orbital Classification}

This section presents several ways in which orbits are classified along with specialized types of orbits. One of the most common ways to categorize an Earth centered orbit is by altitude. Altitude is defined as the distance between the surface of the primary body and the center of the secondary body, whereas the radius of an orbit is defined as the distance between the centers of the two bodies. In the case of Earth orbiting spacecraft, the altitude of a satellite is the mean radius of the Earth $(\sim 6,378 \mathrm{~km})$ subtracted from the orbital radius. The following classifications are based on altitude and are defined in SMAD [50].

- Low Earth Orbit (LEO): The LEO orbital regime includes orbits with an altitude less than $3,000 \mathrm{~km}$, with most being below $1,400 \mathrm{~km}$. The benefits of using a LEO orbit include cheaper launch cost, better resolution for remote sensing, quicker relay times for communications, and faster revisit times. Disadvantages of using a LEO orbit are the smaller viewing area, shorter mission lifetimes due to atmospheric drag, and a large number of satellites are required in a constellation to achieve global coverage. An example of a constellation system in LEO is the Iridium communications constellation.

- Medium Earth Orbit (MEO): The MEO orbital regime includes orbits with an altitude between 3,000 km and GEO (at 35,856 km). GPS constellations are typically placed in MEO orbits just above $20,000 \mathrm{~km}$ and have an orbital period of 12 hours. The advantages of using a MEO orbit are satellites can see a greater area of the Earth than in LEO and will therefore require fewer satellites in a constellation to obtain whole Earth 
coverage. A satellite in a MEO orbit has the disadvantages of requiring more energy to get into orbit, more power to transmit signals, and less resolution compared to LEO satellites.

- Geosynchronous Earth Orbit (GEO): A satellite in GEO has a precise altitude of 35,856 $\mathrm{km}$. At this altitude, the orbital period of the satellite is exactly equal to the period of the Earth's rotation about its axis. The satellite will therefore be orbiting about the Earth at the same rate that the Earth is rotating. If the satellite has an inclination of 0 degrees, it is referred to as Geostationary, and the sub-satellite point will always be over the same point on the Earth. This has a huge advantage as a satellite can cover about $1 / 3$ of the Earth and will always be looking at the same surface. Geosynchronous satellites are typically communications and weather satellites. The disadvantages of using a GEO orbit include poor resolution compared to LEO and MEO, large power requirements to transmit data, and expensive launch costs.

- Super-Synchronous Orbit: A super-synchronous orbit has an altitude above the GEO band but below the moon. The uses for this orbital regime are limited and few satellites exist here.

In addition to classification based on orbital altitude, there are many different specialized orbits that have specific purposes in Earth orbiting space missions. These specialized orbits rely on the use of orbital perturbations to produce specific geometrical patterns and trajectories, and are defined in SMAD [50].

- Repeating Ground Track: In a repeat ground track orbit, an object's sub-satellite point will return to the same location on the surface of the Earth after a certain time frame. Once the object has returned to the same location, it will repeat the same path with a 
certain repeating ground track period. This specific periodicity arises from the orbital period of the spacecraft, where the ground track will repeat after k orbits in $\mathrm{n}$ days, where $\mathrm{k}$ and $\mathrm{n}$ are integers.

- Sun Synchronous Orbit: A sun synchronous orbit relies on the J2 perturbation to cause a rotation in the orbital plane. If the orbit of the satellite is prograde, right ascension of the ascending node will experience a negative regression. Similarly if the orbit is retrograde the regression in the node will be positive. It is therefore possible to maintain a specific combination of altitude and retrograde inclination such that the node will regress by 360 degrees in one year. If this occurs, the orientation of the orbital plane will remain fixed with respect to the Sun as the Earth travels in its orbit about the sun. This has particularly useful applications in remote sensing, where the angle of the sun relative to the Earth and satellite can remain fixed. Additionally, the satellite will cross the ascending node at the equator at the same mean local time in each orbital pass.

- Molniya Orbit: A Molniya orbit utilizes the J2 perturbation to cause the apogee and perigee points to remain constant with respect to the Earth. This occurs when the inclination of the orbit is at 63.4 degrees. In this orientation, the orbital plane will not rotate. This is useful for space systems that provide communications to high latitude regions, where the apogee can be maintained over a local region and provide long dwell times. Soviet communications satellites therefore utilized Molniya orbits to provide long dwell times directly over the former Soviet Union.

- Frozen Orbit: Circular LEO orbits are unstable due to the aspherical nature of the Earth's gravitational potential. Objects in a circular LEO orbit will therefore have small oscillations in eccentricity. In order to negate this often unwanted effect, satellites may 
be placed in a specific orbit with an argument of perigee equal to 90 or 270 degrees and a low orbital eccentricity. In this configuration, the orbit will be stable and the oscillations in eccentricity will be much smaller.

\subsection{Constellation Design}

Now with a brief understanding of orbital mechanics, multiple satellites may be combined to create a working system called a constellation. A satellite constellation is a system

of two or more spacecraft that interact to achieve a common goal. As noted by Wertz [49], constellation design has become a topic of significant interest as constellations become more achievable through smaller and cheaper satellites. Wertz also stated that no set rules exist for constellation design. Companies have invested billions of dollars into LEO communication systems trying to solve the same problem, and have come up with very different solutions. Constellation design is such a difficult problem due to the seemingly infinite design space and solutions that exist. For example, a communications company may wish to achieve $100 \%$ global coverage, while minimizing the number of satellites, and simultaneously minimizing the altitude of the satellites. These design considerations are conflicting in nature and lead to a diverse set of solutions based on diverse requirements.

\subsubsection{Traditional Constellation Patterns}

A multitude of standard design methods and geometries for constellation systems have been created over the past several decades. The most common of these patterns will be discussed here. 


\section{Walker Constellation:}

One of the most notable constellation geometries are Walker constellations, created by John Walker in the late 1960s to the early 1980s [49]. The most common of these constellation designs is the Walker Delta Pattern. The Walker Delta Pattern is a symmetric pattern, where several parameters can specify the design of the entire constellation. The Walker Delta Pattern consists of a total of T satellites, with S satellites evenly spaced in $\mathrm{P}$ orbital planes. Each satellite in the constellation has a common inclination $(i)$, altitude, and occupies a circular orbit. The orbital planes are evenly spaced by ascending node around the equator at intervals of 360/P degrees. Additionally, the satellites in each plane are evenly distributed at intervals of 360/S degrees. Finally, the spacing between satellites in adjacent planes is given by the phasing difference, $\Delta \phi$, which is the phase angle between satellites in adjacent planes. To ensure that all orbital planes have the same relationship with each adjacent plane, $\Delta \phi$ must be an integer multiple, F (between 0 and P-1), of 360/T degrees. The number of satellites in each orbital plane, $\mathrm{S}$, is thereby given as $\mathrm{S}=\mathrm{T} / \mathrm{P}$. The constellation is fully specified with 4 parameters, for a given altitude, written in the notation $i: \mathrm{T} / \mathrm{P} / \mathrm{F}$.

Walker constellations are the most symmetric type of constellation design, and exhibit total symmetry for coverage in longitude. Additionally, due to the characterization of the Walker Delta Pattern, there are a finite number of patterns which may be fully studied.

\section{Streets of Coverage Constellation:}

The streets of coverage design pattern is used to provide $100 \%$ global coverage. In the streets of coverage pattern, there are $n$ satellites in each of the $m$ polar orbital planes. The orbital planes are separated by the distance $\mathrm{D}_{\max }$ at the ascending node, which is given by 


$$
D_{\text {maxs }}=\lambda_{\text {street }}+\lambda_{\text {max }}
$$

where $\lambda_{\text {street }}$ is the half width of the street of coverage, and $\lambda_{\max }$ is the swath half width. Additionally, in order provide continuous global coverage the following equation must be satisfied:

$$
(m+1) \lambda_{\text {street }}+(m+1) \lambda_{\max }>180 \mathrm{deg} .
$$

Figure 2.4 demonstrates the "street" of coverage based on swath width, along with the parameters used to characterize the design.
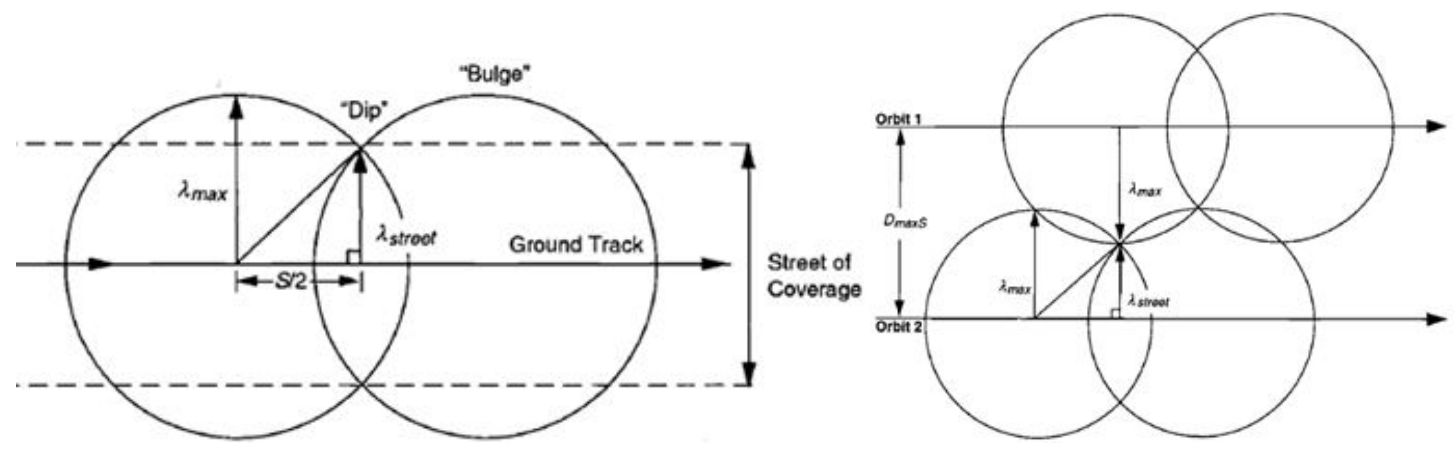

Figure 2.4. Streets of Coverage Pattern. These two diagrams show the basic geometry of the streets of coverage design. The diagram on the left shows the "street" of coverage and the diagram on the right shows the relationship of two orbital planes at maximum separation [49].

With this configuration, the spacecraft are spread out near the equator with little overlap in coverage, but there is great overlap near the poles. Due to the configuration of the pattern, half the satellites will be traveling north while half the satellites will be traveling south at any given point. Two orbital seams will therefore exist where adjacent planes will have satellites traveling in opposite directions. The streets of coverage method is however efficient at producing $100 \%$ global coverage.

\section{Geosynchronous Constellation:}


The simplest form of constellation design is the geosynchronous constellation. This pattern uses several satellites placed in a GEO orbit to achieve a common goal. GEO constellations have the advantage of being able to see a large area of the Earth from such high altitudes, and are therefore used extensively for communications and weather systems. Due to the fact a satellite in GEO can observe roughly $1 / 3$ of the Earth, a few number of satellites are required to perform a given mission. GEO constellation systems perform very well in low and mid latitude regions, but perform poorly over the poles. Most GEO constellations are placed in orbit near 0 degree inclination; GEO constellations may employ high inclination orbits in order to observe higher latitude regions.

\section{Elliptical Orbit Constellation:}

Another type of constellation design utilizes elliptical orbits to introduce an additional degree of freedom which can be useful for providing coverage over specific areas. One of the most popular elliptical orbit constellations is the Molniya constellation. With several spacecraft placed in similar Molniya orbits, continuous coverage over high latitude regions may be obtained which geosynchronous systems cannot provide. Using elliptical orbits, the coverage may be tailored to specific longitude and latitude areas by placing the apoapsis over the desired location. With the apogee placed over the area of interest, a satellite will experience longer dwell times over the specific region. However, elliptical constellations add complexity to the spacecraft design. Elliptical orbits often pass through the Van Allen radiation belts, requiring spacecraft hardware to be radiation hardened.

\subsubsection{Constellation Mission Classification}


Constellation systems may be categorized by the type of mission the entire system is trying to fulfill. The different main types of missions include remote sensing, navigation, and communications. Here a brief overview of these types of constellations along with planned/flown LEO designs will be presented.

\section{Remote Sensing:}

Remote sensing in satellite constellation design is the ability to gather information with the use of multiple satellites through on-board sensors capable of recording electromagnetic radiation. Remote sensing constellation systems have the advantage of being able to view extremely large portions of the Earth at a given time, with very good revisit characteristics. From the standpoint of an entire system trying to capture data of large areas of land, a satellite constellation system is completely unparalleled. Since on-board sensors are acquiring data, usually over the surface of the Earth, it is often necessary to have high ground elevation angles along with the area of interest being illuminated.

Several examples of planned/flown LEO remote sensing constellations include RapidEye, the Disaster Monitoring Constellation for International Imaging (DMCii), GeoEye, Discoverer II, and the Indian Remote Sensing (IRS) constellation. These systems provide both global and regional, high resolution imagery data.

\section{Navigation:}

Navigation constellation systems are one of the greatest technological feats for satellite constellation systems. Navigation constellations allow ground based receivers to determine their exact location with great accuracy. All navigation constellation systems are in a MEO orbit with near 12 hour orbital periods. 
Examples of navigation systems include the United States Global Positioning System (GPS) constellation, the Russian Federation Global Navigation Satellite system (GLONASS) constellation, the China BeiDou constellation, the Indian Regional Navigation Satellite System (IRNSS) constellation, and the planned European Union Galileo constellation. These systems allow ground based receivers to determine their exact location.

\section{Communications:}

One of the major uses of employing multiple satellites in orbit is the ability to relay communications data quickly over extremely long distances. Communications constellations usually employ a great number of satellites in LEO to uplink data from a target on the ground, transfer data between satellites, and downlink the data to another target at a different location on the Earth.

The three main LEO communications constellation systems that are currently operational, with successive generations underway, include Iridium, Globalstar, and Orbcomm. Iridium and Globalstar provide voice and data communications, while Orbcomm provides asset monitoring and messaging communications.

\subsubsection{Constellation Performance Metrics}

This section is intended to provide an understanding of how the performance of a constellation is assessed. Earth coverage is one of the key parameters in assessing constellation performance. The following adapted from Wertz [49], will present basic coverage parameters and notation along with numerical coverage figures of merit. 
Earth coverage is defined as the area of the Earth the satellite payload can "see" over some time frame. Payload here refers to an instrument such as a camera or antenna. The field of view (FOV), or footprint, is the area on the Earth's surface the payload can see at any given instant. Additionally, the access area is the area on the Earth's surface the satellite can see at any given instant. The subsatellite point is the point where the spacecraft radius vector intersects the Earth's surface. A two dimensional ground track is simply a trace of the subsatellite point over time. The spacecraft elevation angle, $\varepsilon$, is the angle between the local horizon and the satellite for a given point on the ground; also called the grazing angle or ground elevation angle. The half cone angle, $\rho$, represents a sensor parameter for conical sensors that defines the half angle of the sensor FOV. Figure 2.5 presents basic Earth coverage geometries and parameters.
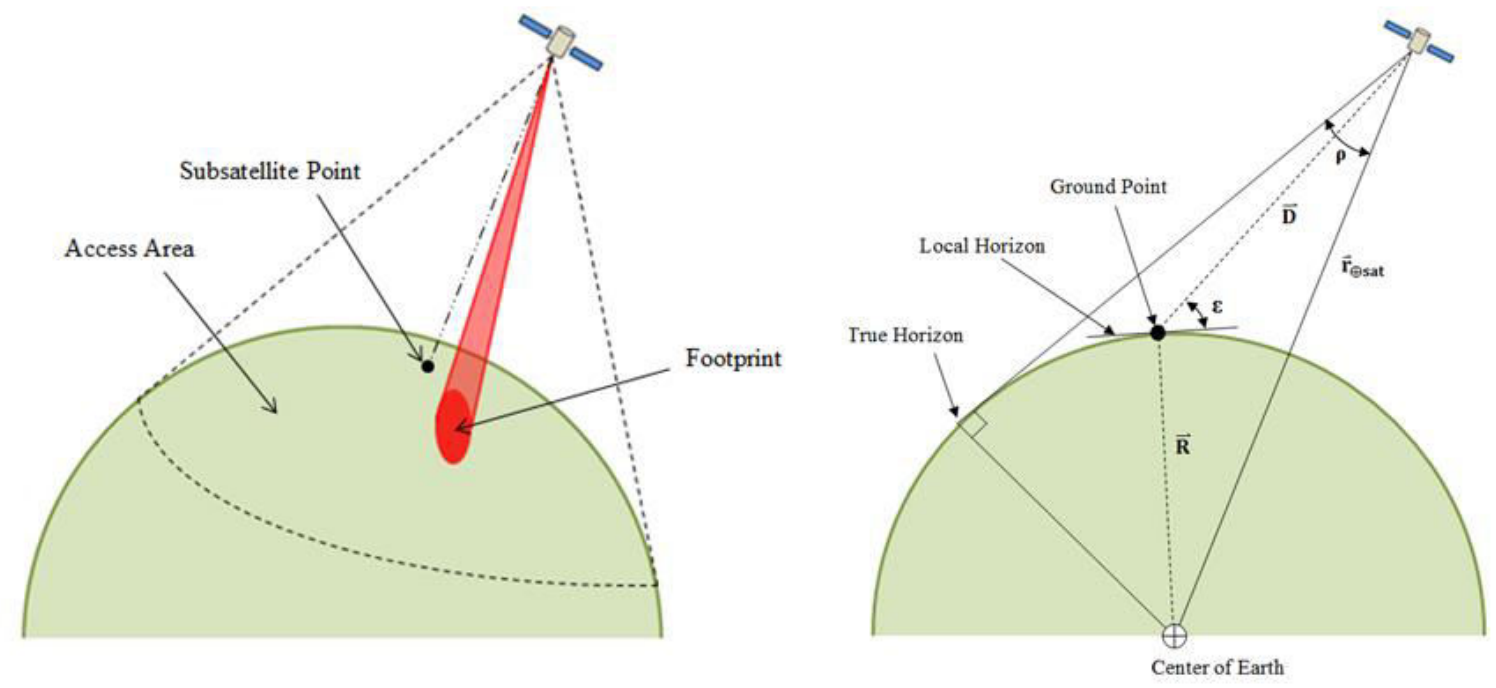

Figure 2.5. Earth Coverage Geometry. The diagram on the left depicts the difference between access area and the satellite footprint; the subsatellite point is also included. The diagram on the right depicts a cutaway view of the Earth with several parameters used to describe coverage. These diagrams are adapted from Wertz [49].

The following formulation provides a simplified analytical approach to calculating the access area. The entire access area on the surface of the Earth is given by 


$$
A_{\text {access }}=2 \pi[1-\sin (\rho)]
$$

where $A_{\text {access }}$ is the access area, and $\rho$ is the angular radius of the Earth. This equation may be modified to determine the footprint area if the payload has a specified FOV. The vector from the satellite to the ground point is given as,

$$
\vec{D}=\vec{R}-\vec{r}_{\oplus s a t}
$$

where the vectors are defined in Fig. 2.5 , and $\vec{D}$ is the slant range vector. The spacecraft elevation angle is then given by,

$$
\varepsilon=\cos ^{-1}(\widehat{R} \cdot \widehat{D})-90^{\circ} .
$$

Using Fig. 2.5, it can be seen that as long as the spacecraft elevation angle is positive, the satellite will be in view to the observer located at the ground point. It is often the case however that buildings or mountains are in the line of sight between the observer and the spacecraft for low elevation angles. A minimum elevation angle may therefore be specified; if the spacecraft elevation angle is greater than the minimum elevation angle, the spacecraft will have access to the ground point. It should be noted that these equations assume a spherical Earth and no obstructions. These equations represent the most basic approach to Earth coverage, and can be further complicated by introducing specific spacecraft instruments with different patterns and requirements.

Performing a numerical simulation is a reliable and effective way to obtain coverage and performance characteristics of a constellation. A grid of ground points can be spread over a desired region, where access between each grid point and each satellite in the constellation is computed at each time step in the simulation. This data can then be converted into meaningful statistical data, such as the average percent coverage of the region of interest over the simulation 
run. Several considerations, as brought up in [49], must be made in order to produce accurate simulation results including grid point spacing, simulation step size, and simulation time span.

The spacing of grid points is extremely important in obtaining accurate data. Ideally, you would like to use an infinite number of grid points in your coverage region, such that every gap in coverage may be recorded and an accurate assessment of percent coverage may be determined. For example, say the region of interest is the entire globe and ten grid points are placed evenly over this region. Ten grid points to cover the entire globe is very few, and as a result through the optimization process, the satellites providing coverage will be optimized to provide coverage over those grid points. The simulation might return data concluding that 100 percent coverage has been achieved. In reality, gaps in coverage might exist where there are no grid points, thereby resulting in an incorrect interpretation of the results. More accurate results will be achieved with more grid points, however more grid points means greater computational complexity and time. Beyond a certain point, adding grid points will not affect the results. The number of grid points to produce accurate results can be determined by performing a grid independence study. The number of grid points is simply increased until adding additional points does not impact the final results. Understanding the resolution and location of the grid points is crucial in providing a correct interpretation of the results.

The simulation time span is also a very important consideration in providing accurate simulation results. Constellation systems provide coverage that is periodic in nature, as the satellites orbit the Earth and the geometry of the system changes. Additionally, gaps may exist at the beginning or end of the simulation run that are not fully recorded. Providing a long enough time span to provide unbiased simulation results due to this periodic nature and gaps in coverage is necessary to produce accurate simulation data. An example given in [49] states that if the 
simulation time span along with the period of the constellation geometry is 24 hours, and there is a 4-hour coverage gap at the beginning of the simulation, then this will be registered as two 2hour gaps. If the simulation is run for a longer period of time however, the gaps in the beginning and end of the simulation will have little impact on the final statistical results.

The simulation step size is similar in nature to the spacing of grid points. If the simulation time step is too large, it is possible that the satellite will miss access between a grid point, and therefore coverage that actually exists will be skipped. The simulation step size must be kept small enough relative to the size of the coverage region and the minimum elevation angle. As the size of the coverage region decreases and the minimum elevation angle increases, the simulation step size must be decreased. As the step size is decreased however, the computational complexity is increased.

Finally, statistical figures of merit must be taken with a grain of salt, as Earth coverage is indeed not statistically distributed in nature. A thorough analysis and understanding of the constellation geometry and the way it provides coverage is necessary to ensure that the statistical data actually represents the real nature of the system. Several figures of merit are often used together in order to better statistically represent the performance of a constellation. Analytical techniques may also be applied to the problem in order to ensure that the statistical data is a good representation of the system. The following coverage figures of merit, discussed in detail in [49], characterize the performance of a constellation, and several of these metrics will be used later in the study.

Gap Time or Revisit Time: Gap time, also known as revisit time, for a single grid point is the length of time when no access to the constellation is reported. This can also be applied to the 
entire coverage grid, where if one or more grid points have no access a gap is recorded. For noncontinuous coverage, there are often multiple gaps during a given time span. Gap time can therefore be broken down into Mean Gap Time and Max Gap Time. Mean gap time is simply the time averaged gap time over the entire simulation run. Maximum gap time is the longest period with no access to one or more grid points over the entire simulation run. If the max gap time over the entire simulation run is 0 seconds, $100 \%$ coverage exists at all times. Max gap time is a useful performance metric because it characterizes what the longest period of no observability will be for all points within the coverage grid.

Daily Visibility Time: Daily visibility time (DVT) is the total time, during a given day, when access exists between the constellation and the grid point. This metric may be applied to the entire grid where DVT may be the total time, during a given day, when access exists between the grid points and the satellites in the constellation. Daily visibility time might be expressed as Minimum DVT, which is the shortest daily visibility time over a multi-day simulation run, or Mean $D V T$, which is the average daily visibility time over a multi-day simulation run. This metric gives an indication of the percent coverage on a daily basis, and therefore gives some insight into the revisit time. 


\section{METHODOLOGY}

This chapter presents an introduction to Multi-Objective Optimization (MOO) and Evolutionary Computing (EC) methods which will be used to obtain the results of this study. An introduction to various optimization techniques will be presented along with a detailed overview of the specific methods that will be used in this study. In addition, the software Systems Tool Kit (STK) and MATLAB will be presented as they are the tools used to perform this work.

\subsection{Multi-Objective Optimization}

Due to the extensive amount of work and literature relating to optimization techniques, a brief overview will be presented here followed by a more detailed review of the chosen method. Multi-objective optimization problems (MOPs) are concerned with obtaining an optimal solution among two conflicting objectives. An example of a MOP for instance might be buying a car. In this case, quality and cost are the two objective functions. Typically, as the quality of the car increases the cost of the car will also increase. You would like to purchase the best quality car for the lowest cost; however these are two conflicting objectives. MOO techniques can therefore be used to find an optimal design or set of optimal designs that find the best tradeoff between high quality and low cost. MOPs exist in nearly all fields of study and a large variety of techniques exist to characterize and solve these problems. MOPs are especially prevalent in engineering due to the conflicting nature of system design; where the four main objectives are performance, cost, risk, and schedule [18].

The goal in multi-objective optimization is to minimize m objective functions,

$$
\vec{F}(\vec{x})=\left[\begin{array}{c}
f_{1}(\vec{x}) \\
f_{2}(\vec{x}) \\
\vdots \\
f_{m}(\vec{x})
\end{array}\right]
$$


with,

$$
\vec{x} \in X
$$

subject to k inequality,

$$
G_{j}(\vec{x}) \geq 0, j=1,2, \ldots, k
$$

and $\mathrm{p}$ equality constraints,

$$
H_{w}(\vec{x})=0, \mathrm{w}=1,2, \ldots, \mathrm{p}
$$

where $\vec{x}$ is the set of solution vectors and $X$ is the set of all feasible solution vectors. For the purpose of this study, all objective functions are considered to be minimization problems; if the objective function needs to be maximized, the sign is simply changed to force a minimization problem.

Different optimization techniques may be classified into the following categories [8]:

- Calculus-Based Methods

- Enumerative Methods

- Guided Random Search Techniques

Calculus-based methods, also known as analytical methods, utilize a set of necessary and sufficient conditions to find the optimal solution to a given problem. Calculus-based methods can be further divided into direct and indirect methods, which have been extensively studied over many years [8]. Direct search methods use hill climbing techniques by moving the solution in a direction related to the local gradient. Indirect search methods use a set of equations based on setting the gradient of the objective function to zero and solving these equations. Both direct and indirect methods require an analytical objective function which must be differentiable. In addition, calculus-based methods provide solutions for local minima as opposed to global minima. The scope of calculus-based methods is therefore limited in practical problems. 
Enumerative methods rely on evaluating the objective function at every point on a discretized search space in order to find the optimal solution. Enumerative methods are simple to implement and can be very efficient for problems with a small search space. Due to the fact that enumerative methods rely on many objective function evaluations, they will break down for problems with complex and large search space.

Finally, guided random search is a stochastic approach to solving MOPs. Guided random search relies on randomization to guide the search space towards an optimal solution. Evolutionary algorithms (EAs) are one of the most popular types of guided random search techniques which will be discussed later. Guided random search techniques are very robust and perform well for problems that have a large search space and are discontinuous in nature [8].

In single-objective optimization, there will be one clear solution that lies at the minimum of the objective function. With multi-objective optimization however, no solution will be optimal in all objectives and thus a solution with the least amount of objective conflict needs to be found. The classical method of dealing with this problem is to weight the objective functions based on importance and sum them together into a single objective function. This now single objective function may be minimized and a single solution will be found. The mathematical relationship of combining the objective functions is given by,

$$
F=\sum_{i=1}^{M} w_{i} f_{i}(x)
$$

where $\mathrm{F}$ is the combined objective function, $\mathrm{M}$ is the number of objective functions, and $\mathrm{w}_{\mathrm{i}}$ is the weight of objective function, $f_{i}$. The sum of all the weights must equal 1. Using the method of objective weighting can be advantageous if knowledge of the importance of objective functions is known. This method however gives no indication of the solution space in multiple objectives which is important to many decision makers. 
Often a better way of obtaining a solution to the multi-objective optimization problem is to find a diverse set of solutions that are said to be Paretooptimal, and then allow decision makers to analyze and choose from among these solutions. A solution that is Pareto-optimal cannot be improved in any objective without degrading another objective. Each Pareto-

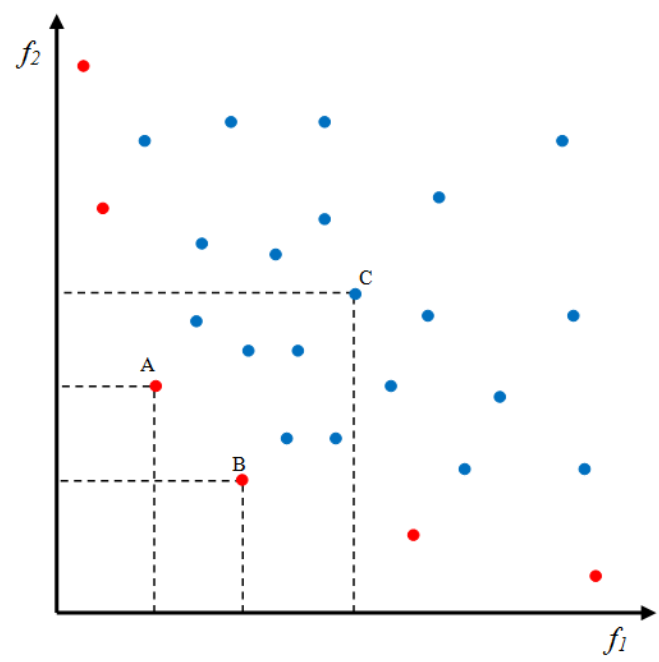
optimal point is therefore equal and is said to be nondominated with respect to each other Pareto-optimal point. If a point is worse in all objectives it is Figure 3.1. Pareto Optimality. This plot demonstrates a Pareto-front along with nondominated and dominated solutions shown in red and blue respectively. Point $A$ and $B$ are both Pareto-optimal and non-dominated with respect to each other, while point $C$ is dominated by both $A$ and $B$.

dominated. Additionally, the set of all Pareto-optimal

points is called the Pareto-front. Figure 3.1 demonstrates a theoretical Pareto-front, with the red points representing Pareto-optimal solutions and the blue points representing dominated solutions; take note the two objective functions are to be minimized. In Fig. 3.1, point A is therefore non-dominated with respect to point $\mathrm{B}$ and vis-versa. Point $\mathrm{C}$ however is dominated by both $\mathrm{A}$ and $\mathrm{B}$ because it is worse in all objectives. Pareto-optimality applies for any number of objective functions that are to be minimized. Formally, if $P$ is the set of Pareto-optimal points, then [29]:

- Any two solutions in $P$ must be non-dominated with respect to each other.

- Any solution not in $P$ is dominated by at least one solution in P.

Additionally, a solution $x_{1}$ dominates another solution $x_{2}$ iff [29],

$$
\begin{gathered}
f_{j}\left(x_{1}\right) \leq f_{j}\left(x_{2}\right) \text { for all } j \in[1,2, \ldots, M], \\
f_{j}\left(x_{1}\right)<f_{j}\left(x_{2}\right) \text { for at least one } j \in[1,2, \ldots, M],
\end{gathered}
$$

where $f_{j}$ is the $j^{\text {th }}$ objective function and $M$ is the total number of objective functions. 
By finding the Pareto-front for a multi-objective optimization problem, the solution space may be analyzed and decision makers may use this information to select an optimal solution from among the front. The Pareto-front's shape helps to show how the objective functions interact as well as show tradeoffs in the solutions. For these reasons, Pareto-frontiers will be developed in this study to show trends in various constellation performance metrics. In order to obtain a Pareto-front, the method of objective weighting may be used by varying the weights and solve for a single Pareto-optimal point in the front many times until the front is full. This is however computationally expensive and may result in a Pareto-front that is not uniform in nature. Multi-objective evolutionary algorithms are very good at solving this type of problem and obtaining a true Pareto-frontier. The goal with these types of algorithms is to minimize computation time, provide a true set of Pareto-optimal solutions, and provide a diverse and uniformly spaced Pareto-frontier.

\subsection{Evolutionary Algorithms}

Evolutionary algorithms are a class of population based, meta-heuristic, global optimization algorithms in artificial intelligence that model the biological mechanisms of evolutionary theory. Evolutionary algorithms are the class of algorithms within the field of evolutionary computing, and the subcategories of EAs include evolutionary programming, evolution strategies, genetic algorithms, and genetic programming. An extremely helpful reference is a book called Introduction to Evolutionary Computing by Eiben and Smith [19]. This resource provides an in depth analysis of the many different aspects to EAs, which will be discussed in detail in this chapter. The main components of EA's are [19]:

\section{- Representation}


- Objective fitness function

- Population

- Parent selection mechanism

- Recombination and mutation

- Survivor selection mechanism

where a specific algorithm has a given order of these components along with a given initialization procedure and termination conditions.

The constellation design space is characterized by an exceptionally large search space that can be continuous/discontinuous, discrete, and nonlinear in nature. Due to the extremely complex nature of the constellation design objective functions, along with the use of numerical simulation to provide the objective functions, calculus-based methods are ruled out. In addition, due to the large search space enumerative methods prove to be much too computationally expensive. Additionally, past research in constellation design utilizing EAs state that enumerative and deterministic techniques prove to perform poorly compared to EAs [20, 41, 42]. EAs provide a stable and robust approach to solving the constellation design problem. Several global optimization alternatives to EAs are Simulated Annealing (SA) and Particle Swarm (PS). Simulated annealing is a probabilistic search algorithm that guides the search towards the area of minimum energy. It does this by simulating the process of annealing in metallurgy to reduce the defects in materials by heating and controlled cooling of the material. Particle swarm optimization is a strategy that guides the search through interaction among individual particles, each with a given position, velocity, and fitness. These three global optimization techniques perform at similar levels, however EAs have been shown to be extremely effective in solving constellation design problems along with being easy to implement $[21,23,24,20,10,38,43,48$, 
$34,6,5,35,36,26,11]$. The most common type of EA, especially in past constellation design research, is the Genetic Algorithm (GA). The genetic algorithm relies on a population of individuals which undergo fitness based selection and genetic operations including recombination and mutation to guide the search. In a GA, the idea of 'survival of the fittest' and environmental pressure guide the individuals in the population toward the optimal solution over a set of generations. The GA has been used for many decades and is very effective at solving problems similar to the problem in this study. For these reasons, it is reasonable that a GA has been selected as the primary tool in this study and will be discussed in more detail in the following section.

\subsection{Genetic Algorithms}

The original genetic algorithm was devised by Holland [19] as a way to study adaptive behavior. It has since been used for decades as an effective way of solving many different optimization problems. Many different variations on the genetic algorithm exist, some of which are quite complex. Figure 3.2 and Fig. 3.3 demonstrate the basic implementation of a GA; the arrangement and implementation of each operator may vary among more complex variations of the GA.

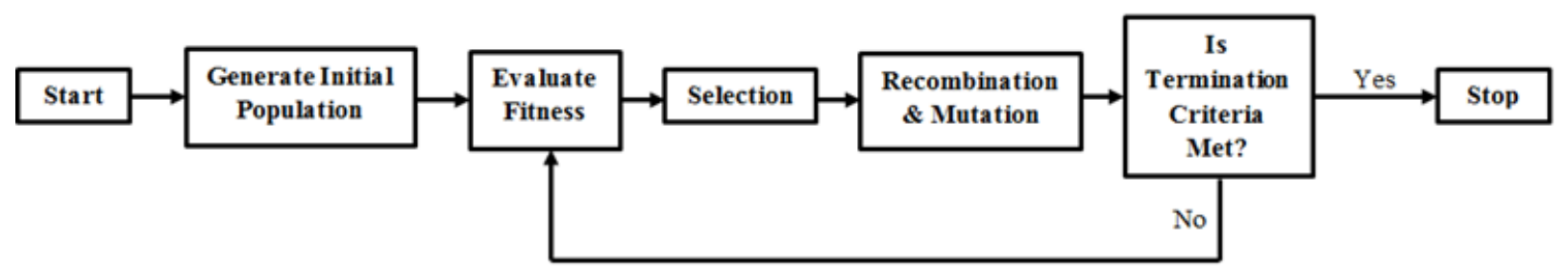

Figure 3.2. Basic Genetic Algorithm Flow Chart. This diagram depicts the flow of a basic GA. 


\section{$B E G I N$}

INITIALIZE population from random potential solutions

EVALUATE individuals and determine fitness

WHILE termination condition is not met

SELECT parents based on fitness

RECOMBINE two parents to create two children

MUTATE the new offspring

EVALUATE individuals and determine fitness

SELECT individuals for next generation END

END

Figure 3.3. Basic Genetic Algorithm Pseudo Code. The above pseudo code, adapted from [19], demonstrates the basic structure of a genetic algorithm.

The algorithm begins with an initial randomized set of potential solutions to the problem called individuals or phenotypes. Each individual has a set of genetic properties called the chromosomes or genotype. Each individual therefore has two main properties associated with it, the phenotype and the genotype. The phenotypic properties are those that are expressed on the outside, while genotypic properties are expressed on the inside as the genetic makeup of the individual; the individual's genotype is said to encode its phenotype. The chromosome is made up of multiple genes that determine the phenotypic properties of the individual. It is possible and also likely that a single gene affects more than one phenotypic property, and a single phenotypic property may be affected by multiple genes. Care must be taken when deciding how to represent the phenotype in the chromosome.

After the initial population is randomly generated, the iterative process begins by evaluating each individual's fitness using the objective functions. The fitness or objective function is used to rank individuals based on how well they conform to the environment or requirements of the problem being solved. Next, selection takes place where parent individuals are selected based on a stochastic fitness-based selection scheme. In this process, an individual 
has a higher chance of being selected if it has a higher fitness value. Once two parents are selected, the genetic operations take place. Recombination involves performing crossover on the two parent individuals to produce two children individuals. Recombination occurs on the genotypic level, where the chromosomes of the two parents are effectively mixed to produce two children chromosomes that are made from parts of the parents. Two parents are typically used to produce two children until the child population is the same size as the parent population. Mutation then takes place where each gene of each child chromosome has a certain chance of being mutated, or altered. Next, the termination criteria for the iterative process of the algorithm are assessed. If the termination criteria are met, the algorithm is stopped and the final population is given. If the termination criteria are not met, the algorithm continues the iterative process by evaluating the child population and selecting new parents from the child population. The process described above is the basic algorithm for a GA. Many complex and novel algorithms exist which greatly improve the performance of the GA. Some of these improved algorithms will be discussed in future sections, along with the specific algorithm used in this study.

Now that the basic algorithm is defined, more detail will be provided about the basic operators involved in the algorithm as detailed in [19]. Optimizing the orbit of a single satellite will be used as an example to help better understand the GA process.

\section{Representation:}

The process of representation is how the real world problem is linked to the world of the EA. As stated earlier, care must be taken when deciding how to represent the phenotype as part of the chromosome. The genotype is said to encode the phenotype, and there are several different ways of encoding of the phenotypic variables as part of the chromosome. These 
options include binary, integer, real valued, and permutation representations. Consider a satellite in orbit that is represented fully by the six COEs: $1000 \mathrm{~km}, 0,45 \mathrm{deg}, 230 \mathrm{deg}, 60 \mathrm{deg}$, and 100 deg. The phenotype for this example would be the six COEs which represent the geometry of the satellite in orbit. The phenotype would then be mapped to a genotype which would be either a binary, integer, real valued, or permutation representation. If a binary representation is used, the six COEs would be mapped from their real values into a binary form. The chromosome would then take on a string of ones and zeros that make up the six COEs or genes as follows:

$$
\underbrace{1111101000}_{a} \underbrace{0}_{\text {ecc }} \underbrace{101101}_{\text {inc }} \underbrace{11100110}_{\omega} \underbrace{111100}_{\Omega} \underbrace{1100100}_{\theta} \text {. }
$$

Binary representation is the earliest form of representation used in a GA and is very simple to use.

Integer representation can be useful to map a genotype to a phenotype that is restricted to a finite set or represents non-number phenotypes such as words. For example mapping a set of directions could be done by letting the values [1, 2, 3, 4] represent [Left, Right, Up, Down].

Real valued representation is similar to binary representation, and is often the simplest method if the solution space is a set of continuous real values. For the example of a satellite with the six earlier defined COEs, the chromosome would take on a string of real values as:

$$
\underbrace{1000}_{a} \underbrace{0}_{\text {ecc }} \underbrace{45}_{\text {inc }} \underbrace{230}_{\omega} \underbrace{60}_{\Omega} \underbrace{100}_{\theta} \text {. }
$$

With real valued representations, it is also possible to let all genes be expressed as any real number on the set $[0,1]$. A mapping is then created that allows the transformation between a gene with bounds of $[0,1]$ and the real problem value. For example if the inclination is allowed to vary between 0 and 180 degrees. The genotypic value may vary from 0 to 1 , and the inclination is given by the genotypic value multiplied by 180 . With this mapping, care must be taken to allow a large enough precision in the genotypic values since they are usually represented 
on a smaller set than the phenotypic values. For example, if the inclination is needed at a precision of four decimal places, the genotype for inclination would need to have six decimal places since it is two orders of magnitude smaller than the phenotype. Finally, the genetic operators will be performed on the genes that are expressed on the set $[0,1]$, and when the fitness evaluation takes place the genotypic values will be transformed to the real world set via the mapping operator.

Finally, permutation representations are useful for problems in which the decision is placed on the order in which something occurs, such as optimizing directions or an order of events. Care must be placed on deciding the genetic operators, since integers in the permutation cannot occur more than once.

\section{Selection:}

In order to produce a new generation of solutions in a GA, parents must be selected for mating in order to produce a child population. A variety of selection strategies exist which will be discussed here.

Fitness proportional selection, also known as roulette wheel selection, is a method where an individual has the probability of being selected based on its fitness compared to the fitness of the entire population:

$$
\frac{\vec{x}_{i}}{\sum_{j=1}^{N} \vec{x}_{j}}
$$

where $\vec{x}_{i}$ is an individual in the population and $\mathrm{N}$ is the number of individuals in the population. This was one of the first selection methods introduced, but it does carry several problems. If several individuals have much higher fitness than the rest of the population, the population may become overrun with these solutions causing premature convergence. In addition, when fitness 
values for all individuals in the population are very similar, worse individuals have nearly the same chance as better individuals of being selected. This means there is effectively no selection pressure and the algorithm will progress very slowly.

Rank based selection corrects several of the limitations of fitness proportional selection. The individuals in the population are sorted based on fitness and then assigned a selection probability based on the rank of the individual. The selection probability can be applied to the rank of an individual in either a linear or exponential fashion. With a linear scheme, the selection pressure is very little, but with exponential schemes, the selection pressure can be varied based on the shape of the exponential curve.

Tournament based selection provides a simple implementation and effective way of creating the mating pool. In tournament based selection, $\mathrm{k}$ individuals are selected to go into a tournament pool. The individual with the best fitness in the tournament pool is selected as a parent. This is performed iteratively until the mating pool is full. The size of the tournament pool, $\mathrm{k}$, controls the amount of selection pressure. This method is widely used in GAs today due to its simplicity and effectiveness.

\section{Recombination:}

Recombination, or crossover, is a genetic operator which creates a set of child chromosomes from a set of parent chromosomes. Recombination is the primary method of introducing genetic diversity into the population. Many different methods of recombination exist for different types of representations. The most common methods for binary and real-valued representations will be discussed here. 
For binary representations, the standard types of crossover include single-point crossover, n-point crossover, and uniform crossover. Single-point crossover is the standard crossover method used in binary recombination. A random crossover point is selected in the binary chromosome string. The bits to the left of the point are copied from parents 1 and 2 to children 1 and 2 respectively, while the bits to the right of the point are copied from parents 1 and 2 to children 2 and 1 respectively. Single-point crossover is depicted in Fig. 3.4. Similar to singlepoint crossover is n-point crossover, where multiple crossover points are selected and alternating sections of genetic material are swapped between the parents to produce the child chromosomes. N-point crossover is depicted in Fig. 3.5. Finally, uniform crossover presents each bit with an equal chance of being inherited from either parent. For each bit location, there is a $50 \%$ chance the child will inherit the bit from parent 1 and a 50\% chance the child will inherit the bit from parent 2. The first child is created based on this uniform crossover mechanism, and the second child is simply the inverse of the first child. Uniform crossover is depicted in Fig. 3.6.

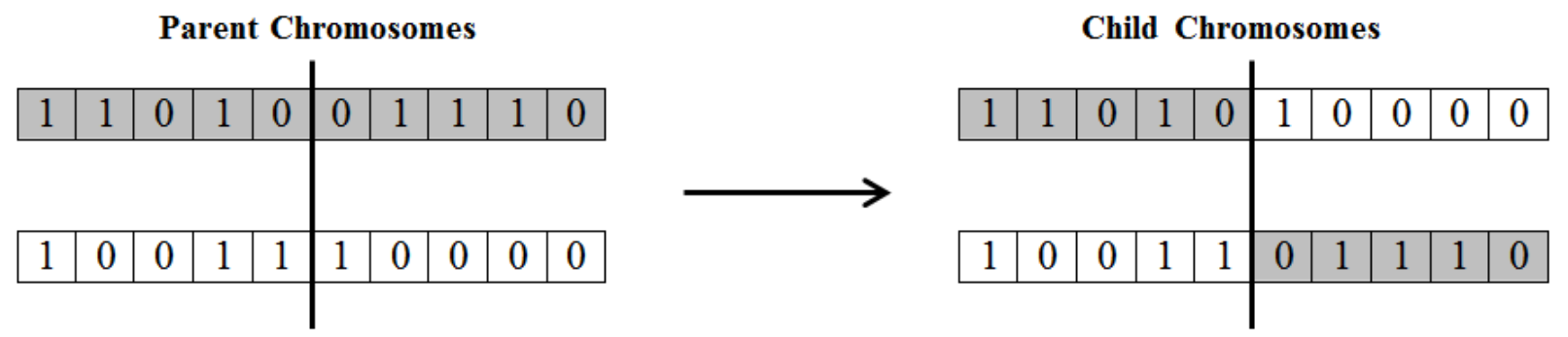

Figure 3.4. Single-Point Crossover. This diagram depicts the mechanism of single-point crossover for a binary representation of a chromosome. Note that the gray shaded bits are from parent 1 and the white shaded bits are from parent 2. 

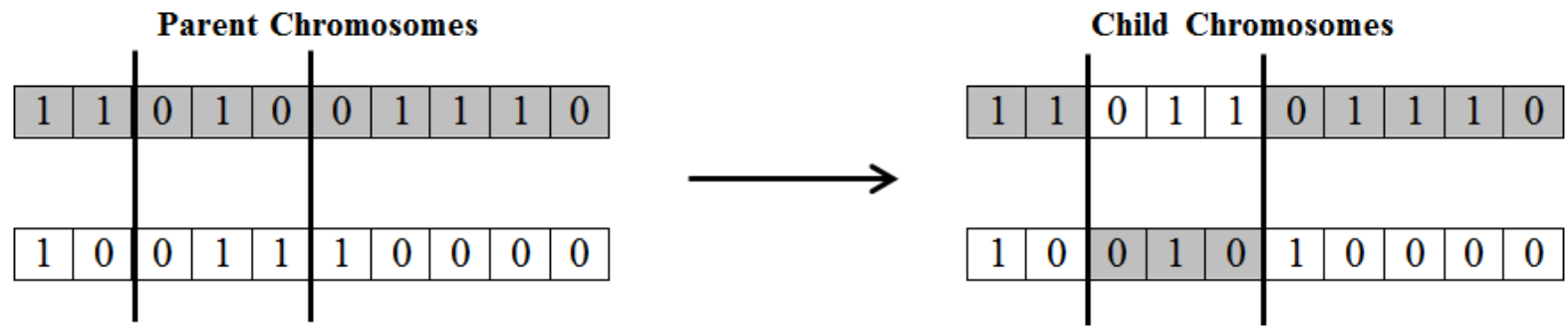

Figure 3.5. N-Point Crossover. This diagram depicts the mechanism of n-point crossover for a binary representation of a chromosome. Note that the gray shaded bits are from parent 1 and the white shaded bits are from parent 2. In this case, $n$ is equal to two.

Parent Chromosomes

\begin{tabular}{|l|l|l|l|l|l|l|l|l|l|}
\hline 1 & 1 & 0 & 1 & 0 & 0 & 1 & 1 & 1 & 0 \\
\hline
\end{tabular}

\begin{tabular}{|l|l|l|l|l|l|l|l|l|l|}
\hline 1 & 0 & 0 & 1 & 1 & 1 & 0 & 0 & 0 & 0 \\
\hline
\end{tabular}

Child Chromosomes

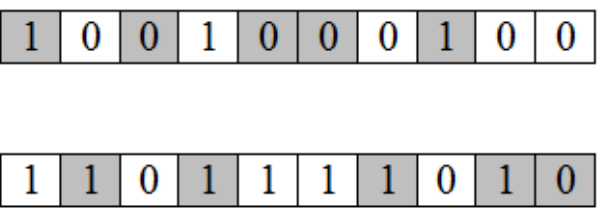

Figure 3.6. Uniform Crossover. This diagram depicts the mechanism of uniform crossover for a binary representation of a chromosome. Note that the gray shaded bits are from parent 1 and the white shaded bits are from parent 2. In this case, there are no crossover points, but rather each bit has a chance to come from either parent. Note that child 2 is simply the inverse of child 1.

For real-valued representations, the most common forms of recombination are simple arithmetic, single arithmetic, and whole arithmetic. Simple arithmetic recombination involves picking a crossover point along the chromosome. The values to the left of the crossover point are taken directly from parent 1 and placed into child 1 and directly from parent 2 and placed into child 2. The values to the right of the crossover point are the arithmetic average of the two parents given by,

$$
\begin{aligned}
& \text { Child 1 }=\alpha \cdot x_{i}+(1-\alpha) \cdot y_{i} \\
& \text { Child 2 }=\alpha \cdot y_{i}+(1-\alpha) \cdot x_{i}
\end{aligned}
$$

where $x_{i}$ represents the $i^{\text {th }}$ bit of parent $1, y_{i}$ represents the $i^{\text {th }}$ bit of parent 2 , and $\alpha$ is a value in $[0,1]$. For simple arithmetic recombination, in Eqn. 3.9 and 3.10 the $i^{\text {th }}$ term would be for every term to the right of the crossover point. In single arithmetic recombination, instead of picking a 
crossover point, only a single gene is averaged using Eqn. 3.9 and 3.10. In whole arithmetic recombination, every gene in the entire chromosome is the arithmetic average of the two parent chromosomes, such that in Eqn. 3.9 and 3.10, the $\mathrm{i}^{\text {th }}$ term would be for every single gene. Figures 3.7, 3.8, and 3.9 and demonstrate these three methods of recombination.

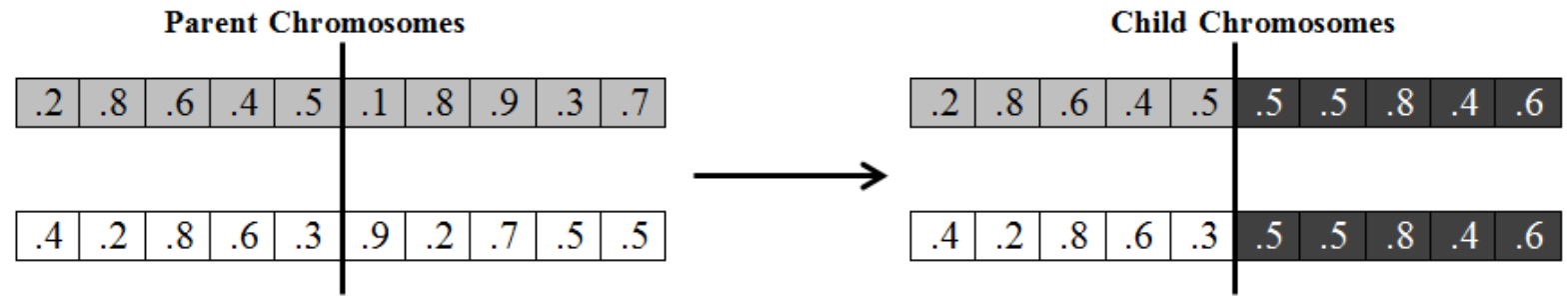

Figure 3.7. Simple Arithmetic Recombination. This diagram depicts the mechanism of simple arithmetic recombination for a real valued representation of a chromosome. Note that the light gray bits are from parent 1 , the white bits are from parent 2 , and the dark gray bits are an average of parent 1 and 2 . In this case, $\alpha=.5$.

Parent Chromosomes

Child Chromosomes

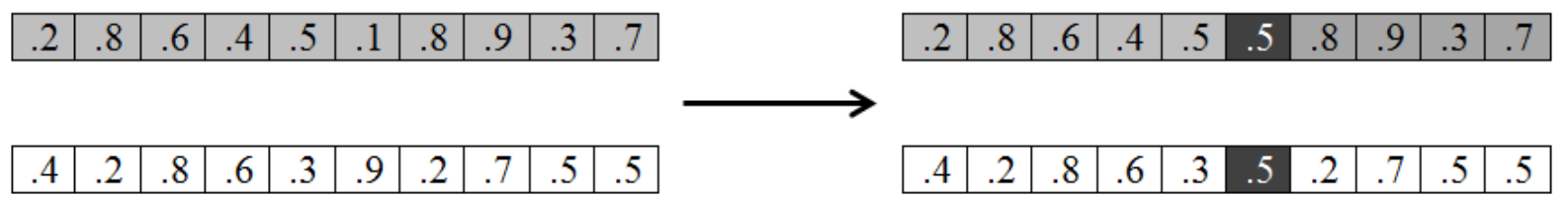

Figure 3.8. Single Arithmetic Recombination. This diagram depicts the mechanism of single arithmetic recombination for a real valued representation of a chromosome. Note that the light gray bits are from parent 1 , the white bits are from parent 2, and the dark gray bit is an average of parent 1 and 2. In this case, $\alpha=.5$.

Parent Chromosomes

\begin{tabular}{|l|l|l|l|l|l|l|l|l|l|}
\hline .2 & .8 & .6 & .4 & .5 & .1 & .8 & .9 & .3 & .7 \\
\hline
\end{tabular}

Child Chromosomes

\section{\begin{tabular}{l|l|l|l|l|l|l|l|l|l|l|}
.3 & .5 & .7 & .5 & .4 & .5 & .5 & .8 & .4 & .6
\end{tabular}}

Figure 3.9. Whole Arithmetic Recombination. This diagram depicts the mechanism of whole arithmetic recombination for a real valued representation of a chromosome. Note that the light gray bits are from parent 1 , the white bits are from parent 2, and the dark gray bits are an average of parent 1 and 2 . In this case, $\alpha=.5$. Note how the children chromosomes lie within the area of the parent due to an $\alpha$ value of.5.

It should be noted that a potential drawback of these methods is that since $\alpha$ is a value in $[0,1]$, the potential area of the offspring is restricted to inside the area of the parents. This is 
evident in Fig. 3.8 as the offspring chromosomes are all within the bounds of the parents. Over time, the area will shrink due to the fact that child chromosomes won't be generated on the border of this area. In order to overcome this, a new set of $\alpha$ values are used where,

$$
\alpha \in[-d, 1+d]
$$

where a value of $\mathrm{d}=0.25$ statistically

ensures that over time the possible area of the children is the same as the possible area of the parents [39]. One interesting method similar to
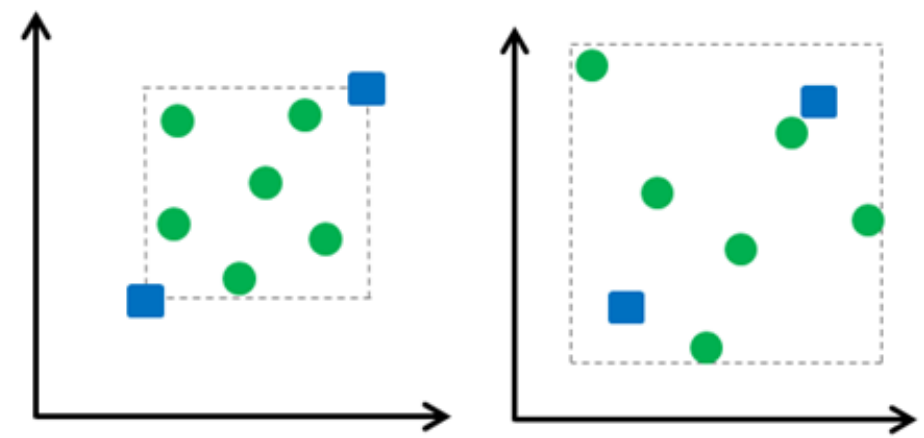

whole arithmetic recombination is to

Figure 3.10. Potential Area of Children from Two Parents. These two plots depict the potential area of the children(green select a new random value of $\alpha$ for circles) based on the two parents(blue squares). The plot on the left is based on a value of $d=0$ in Eq. 3.6. The plot on the right is a each gene on the space spanned by hypercube based on a value of $d=.25$ adapted from [ (Pohlheim 2007)].

Eqn. 3.11. It is possible with this

method that a gene will be generated that lies outside the possible area for which a solution may exist, in which case the solution will be disregarded and a new value of $\alpha$ will be randomly determined. This means that the area spanned by the children will be a hypercube that is a little larger than the parents, as depicted in Fig. 3.10.

Many other methods of recombination exist for both binary and real valued representations. For permutation representations the common methods of recombination are partially mapped crossover, edge crossover, order crossover, and cycle crossover. For integer representations, variations on the previously described methods may be used. For more detailed explanations on these methods of recombination, see [19]. 


\section{Mutation:}

Mutation is the secondary method of introducing new genetic material into the population. Without mutation, the algorithm may prematurely converge due to a lack of new genetic material. The process of mutation involves making extremely small changes to the genetic material. Many different methods of mutation exist, and the most common methods for binary and real valued representations will be discussed here.

The most common method of mutation for binary representations is bit flipping. Using this technique, each bit in the chromosome string is subject to a bit flip (changing 1 to 0 or 0 to 1). The probability that a bit flip will occur for each bit in the string is $p_{m}$ and is usually very small. A standard value for $\mathrm{p}_{\mathrm{m}}$ is $1 / \mathrm{L}$, where $\mathrm{L}$ is the number of bits in the chromosome string; this makes it so that on average 1 bit will be flipped for each chromosome string. By changing a single bit, the value for a given gene is thus altered and new genetic material is introduced into the population. Figure 3.11 depicts a bit flip mutation for a chromosome string.

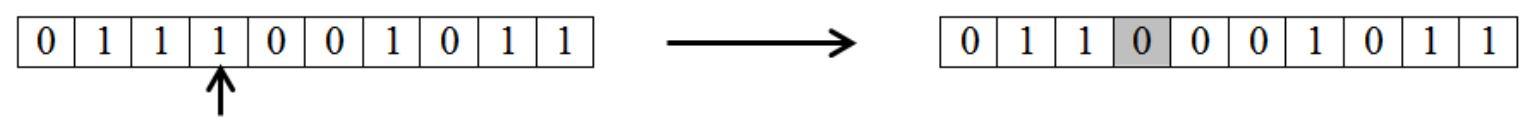

Figure 3.11. Bit Flip Mutation for Binary Representation. This image depicts a single bit flip in a chromosome string. In this case, the $4^{\text {th }}$ bit in the string was selected for mutation and was changed from a 1 to a 0.

The two types of mutation techniques for real valued representation are uniform mutation and non-uniform mutation. In uniform mutation, each gene has a probability of being mutated, $\mathrm{p}_{\mathrm{m}}$. It is common practice to let $\mathrm{p}_{\mathrm{m}}$ in this case to be equal to $1 / \mathrm{L}$ where $\mathrm{L}$ is the number of genes in the chromosome string. When a gene is selected for mutation, a new value for the gene is randomly created from the entire space in which the gene exists. In non-uniform mutation, the probability for selection is the same, but the mutation operator is based on a Gaussian distribution instead of a random alteration. Non-uniform mutation works by adding a value to 
the gene that is randomly selected from a Gaussian distribution with a mean of zero and a defined standard deviation. This ensures that most of the time small changes are made in either direction, with the possibility of large changes being made. The larger the standard deviation, the higher the chance of making relatively larger changes to the gene. It is possible to alter the gene beyond the limits of the space in which the gene is allowed. If this occurs, depending on the algorithm, the gene may either be truncated to the boundary or the mutation operator is applied again until a value is found within the boundaries.

\subsection{Multi-Objective Genetic Algorithms}

This section presents the various multi-objective genetic algorithms (MOGAs) that are being used today, along with a detailed overview of the algorithm used in this study.

\subsubsection{Variations on the Multi-Objective Genetic Algorithm}

Common Pareto-based evolutionary algorithms include the Strength Pareto Evolutionary Algorithm (SPEA) [51], Strength Pareto Evolutionary Algorithm 2 (SPEA2) [52], NonDominated Sorting Genetic Algorithm (NSGA) [17], Non-Dominated Sorting Genetic Algorithm II (NSGA-II) [15], Pareto Archived Evolution Strategy (PAES) [31], Vector Evaluated Genetic Algorithm (VEGA) [40], Non-Dominated Rank Based Sorting Genetic Algorithm (NRSGA) [29], along with epsilon dominance ( $\varepsilon$ ) variations on these algorithms. Several studies exist [32] which compare these different algorithms, of which the eNSGA-II performs consistently well. Several studies described in the Introduction are successful in using the $\varepsilon$ NSGA-II algorithm for similar types of constellation design problems. For these reasons a variation on the $\varepsilon$ NSGA-II will be used which is discussed in detail in the following section. 


\subsubsection{Epsilon Non-Dominated Sorting Genetic Algorithm II (ENSGA-II)}

The algorithm used in this study to obtain the various Pareto-frontier plots is most similar to the eNSGA-II algorithm created by Deb in [15], with modifications by Kollat and Reed in [33], modifications by Ghosh and Das in [29], and additional modifications to by the author to better fit the algorithm to the problems in this study. In order to reduce repetition, the modified algorithm used in this study in its entirety will be presented here.

Firstly, the idea of epsilon-dominance will be presented. Earlier in Section 3.1, the ideas of Paretodominance and the Pareto-frontier were discussed. The final goal of a Pareto-based EA is to produce an optimal, well-spaced, and diverse Pareto-frontier. If the objective space is continuous in nature, it is possible that an infinite number of points will exist along the Pareto-frontier. Traditional EAs ran into the problem that if all of the never-dominated solutions are stored in an archive, the

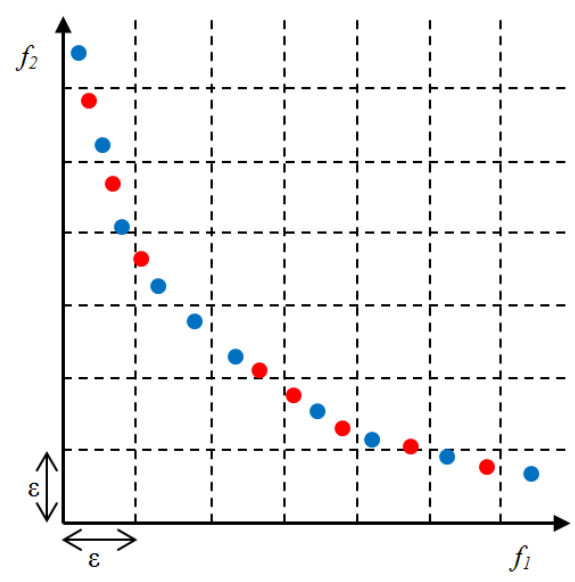

Figure 3.12. Epsilon-dominance. This plot depicts a Pareto-frontier of points, where the red points are solutions that are removed using the epsilon-dominance technique. The blue points are the solutions that will remain. archive will grow without bound and searching or adding to the archive would become too computationally expensive. By thinning out the Pareto-frontier, the user has the ability to specify the resolution of the final frontier. Additionally, convergence will occur with fewer function evaluations with a smaller Pareto-frontier and population size. One very successful method of thinning the Pareto-frontier is the idea of epsilon-dominance. Epsilon-dominance is depicted in Fig. 3.12. A grid is placed over the objective space, with grid spacing determined for 
each objective, where only one solution may exist within each grid block. If two or more solutions exist within a grid block, the solution closest to the minimum point in the grid block is chosen. A modification to the traditional epsilon-dominance function in this algorithm is that two solutions are allowed to exist in the first, or minimum, grid block in each objective function. This allows the absolute minimum solution of each objective to be retained instead of being deleted for a solution that is slightly larger than the true minimum. The epsilon-dominance function is applied to the offline archive which will be discussed shortly.

Figure 3.13, from [32] depicts the basic structure of the $\varepsilon$ NSGA-II algorithm.

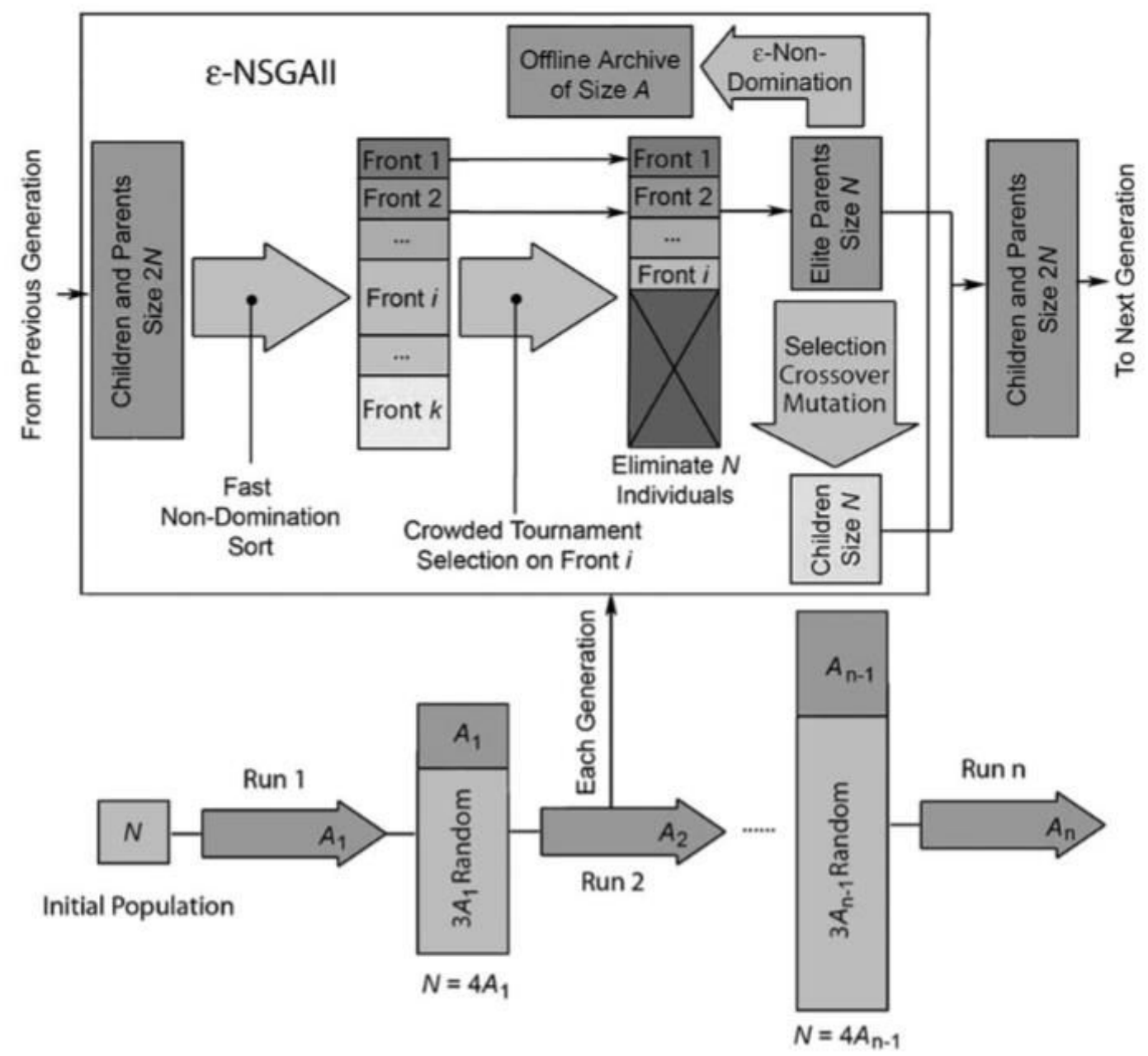

Figure 3.13. Diagram of the ENSGA-II algorithm as shown in [32]. This diagram depicts the basic structure and flow of the algorithm that is used in this study. The series of connected runs, dynamic population sizing, and offline archiving of the Pareto-frontier is shown. 
Now that the idea of epsilon-dominance has been presented, the structure of the algorithm used in this study will be discussed. The basic flow of the \&NSGA-II algorithm is as follows:

- An initial parent population of $\mathrm{N}$ individuals is randomly created.

- The fitness of the parent population is computed and an adjusted fitness value is given to each individual based on non-dominated fronts, rank, and crowding distance. The individuals that are non-dominated in this initial population are added to an offline archive.

- A child population of size $\mathrm{N}$ is then created from the parent population using the selection, recombination, and mutation operators.

- The fitness of the child population is computed.

- The parent and child populations are then merged into a single population of size $2 \mathrm{~N}$. The merged population is classified and each individual is given an adjusted fitness value. The worst $\mathrm{N}$ individuals are eliminated from the merged population to create a new elite population of $\mathrm{N}$ individuals.

- This elite population is then compared to the offline archive, and individuals in the elite population are added to the archive based on non-dominance. Epsilon-non-domination is applied to the archive.

- Next, the elite population becomes the parent population for the next generation and the process is repeated.

- Once the algorithm fails to produce a given number of additions to the offline archive in a single generation, the archive is used to seed a new run of the algorithm. The entire archive represents $25 \%$ of the new initial population and $75 \%$ of the individuals are randomly generated. 
- The series of connected runs are then performed until the Pareto-frontier is obtained.

Now that the basic structure of the algorithm has been presented, a more detailed analysis of the structure and operators used in this study will be presented. The initial size of the first random population is $\mathrm{N}=5$ individuals. By starting with such a small population size, the algorithm is able to quickly progress towards the optimal Pareto-frontier. Past work [12] along with studies performed in this work have shown that by starting with a small population of $\mathrm{N}=5$ individuals, faster convergence is achieved. The initial population is randomly generated, by creating 5 individual chromosomes. For a Walker constellation study, seven genes make up the entire chromosome of an individual consisting of constellation altitude, constellation inclination, seed right ascension of the ascending node, seed mean anomaly, number of spacecraft, number of planes, and the phasing between satellites in adjacent planes. A real valued representation is selected for this study, due to the continuous nature of the phenotype. A real valued representation is also selected due to the simplicity in performing the genetic operations and altering the chromosome structure between case studies. In order to provide simplicity, along with uniform resolution for the genetic operators between genes, each gene is scaled to exist on the set $[0,1]$. A mapping therefore exists in order to convert the real valued genotypic representation between 0 and 1 to the actual phenotypic values. For instance, the inclination on the genotypic level is expressed as a value between 0 and 1 . To convert this to a phenotypic value, the genotypic value is simply multiplied by 180 in order to obtain an inclination value that is bounded by $[0,180]$ degrees. The following depicts how the chromosome is structured as a vector,

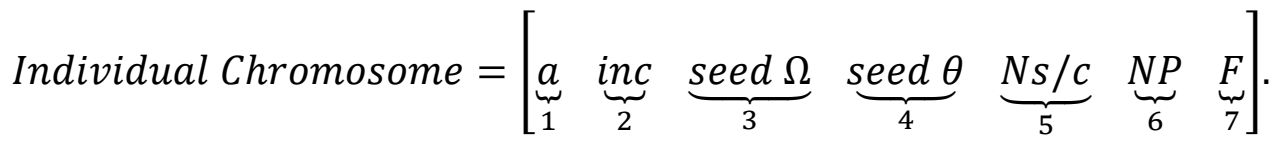


The random initial population is therefore a $5 \times 5$ matrix, with each row representing an individual chromosome and each column representing a single gene. The initial population is simply created by generating a random 5x5 matrix, with each value a random value between 0 and 1 .

In this study, the fitness of an individual is performed by numerical simulation in STK, which will be discussed in more detail in a later section. Once the fitness has been computed, an adjusted fitness value is given to each individual based on several factors. First, the population is classified into fronts based on non-domination. All of the individuals in the population that are non-dominated are placed into front 1 . The solutions in front 2 are then all of the non-dominated solutions in the population with the individuals from front 1 removed. All of the solutions are classified into fronts using this same iterative method. Next, a rank is assigned to each individual where the rank is given by [29],

$$
\operatorname{rank}_{i}=n_{i}+1
$$

where $n_{i}$ is the number of solutions that dominate solution

$i$. Next the density of the region in the objective space surrounding each individual is computed based on a crowding distance function given by Deb in [16]. The crowding distance for each individual, $d_{i}$, is a measure of the perimeter of the cuboid that is formed by the vertices of the nearest solutions in the objective space. Figure 3.14 provides an illustration of the cuboid in a twodimensional objective space. The crowding distance is

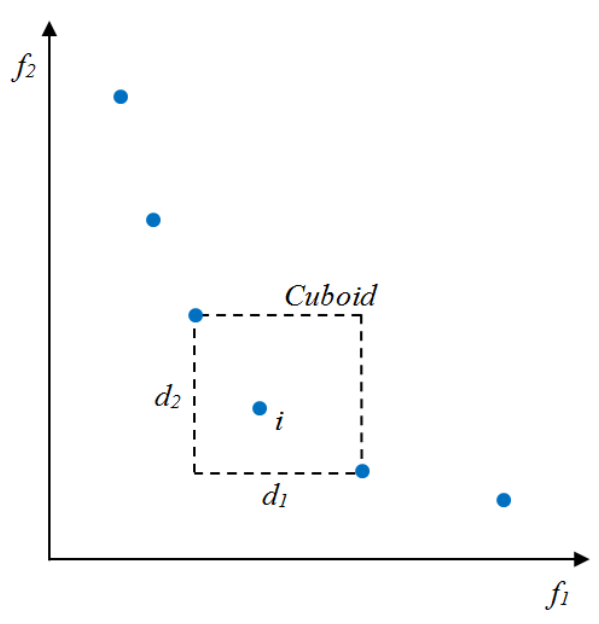

Figure 3.14. Crowding Distance Cuboid. This plot, adapted from [16], depicts the cuboid formed by the vertices of the nearest solutions to $i$ in the $2 D$ objective space. simply the distance between consecutive solutions in a given objective, normalized by the span 
of solutions in that objective, and summed across all objectives. The crowding distance for an individual, in reference to Fig. 3.14, is given explicitly as the sum of distance $d_{1}$ and $d_{2}$ normalized by the total span of the solutions in objective 1 and objective 2 respectively. This function is applicable to any number of dimensions by simply summing these distances for each objective. The solutions that lie on the end of each objective are assigned an infinite crowding distance. Once the front, rank, and crowding distance for each individual in the population are determined, the adjusted fitness is computed as outlined in [29].

- Each individual in front 1 is assigned an average fitness value equal to the number of individuals in the population; $F_{\text {avg }}=N$ for the individuals in front 1.

- Each individual in the current front is then assigned an adjusted fitness which is computed from the average fitness and the rank of the individual; $F_{a d j}=F_{a v g}-g$, where $g$ is the number of individuals in the same front that have a rank less than or equal to the given individual.

- The final fitness value for each individual in the current front, $F$, is computed from the adjusted fitness value and the crowding distance for each individual; $F=F_{a d j}-1 / d$, where $\mathrm{d}$ is the crowding distance for a given individual.

- If $F_{m}$ is the minimum fitness of an individual $m$, and $F_{m}$ is negative, then $0-F_{j}$ is added to the fitness of all individuals in the population to make the fitness of all individuals positive.

- This process is repeated for each front until every individual in the population is assigned a new fitness value, $F$. The average fitness value, $F_{a v g}$, for the solutions in the next front are given as the minimum fitness value, $F$, from the previous front minus a small positive 
value. This ensures all individuals in front 2 will have a fitness that is worse than the individuals in front 1 , and so on for all fronts.

It should be noted that although the objective fitness is to be minimized, a larger fitness value, $F$, corresponds to a more fit individual. This approach of applying the front, rank, and crowding distance to each individual in the population to produce a single fitness value helps to apply selection pressure to individuals that are less dominated and that are in less crowded regions of the objective space.

The specific genetic operators used are given as follows:

- The selection operator is tournament based selection. Two parent individuals are randomly selected, and the individual with the higher fitness value, $F$, is selected for mating. This occurs twice to obtain two parent individuals which will be used to generate two child individuals.

- The recombination operator is whole arithmetic recombination, as shown in Fig. 3.9. A new alpha value is selected for each gene as given in Eqn. 3.11, with $\mathrm{d}=0.25$.

- The mutation operator is non-uniform mutation. The chance of a gene being selected for mutation is given as $1 / N_{\text {gene }}$, where $N_{\text {gene }}$ is the length of the chromosome given by the number of genes. Statistically, over time each chromosome will have one gene mutated on average. The standard deviation used in the Gaussian distribution is 0.25 .

Additional properties of this algorithm are the use of elitism, an offline archive, time continuation, and auto-adaptive population sizing. Elitism is the idea that the parent individuals have a chance at becoming members of the population in the next generation. This ensures that if the parent population produces a child population that is less fit, the search will not regress to a 
worse population. The use of an offline archive is necessary in order to save solutions that are in the Pareto-frontier, maintain well spread solutions through epsilon-domination, and have a way of measuring how well the algorithm is performing. Additionally, time continuation is utilized by adding new random solutions to the population once the algorithm fails to produce new solutions to the archive. Time continuation helps to periodically progress the search and prevents a poor random initial population from limiting the search from finding the true global optimums. Finally, auto-adaptive population sizing stems from the fact that the population size is directly proportional to the size of the archive. The population size will be four times the size of the archive, except in the first run. This eliminates the need to manually change or set the size of the population. The changing size of the population will not waste computational resources when the archive is very small, and the algorithm will not be underutilized when the archive is very large.

\subsection{Algorithm Verification}

The algorithm was written in MATLAB and several mathematical benchmark problems were used to test the algorithm. The benchmark problems used were the Viennet function along with ZDT1, ZDT2, ZDT3, ZDT4, ZDT6, SCH, and FON found in [15]. 


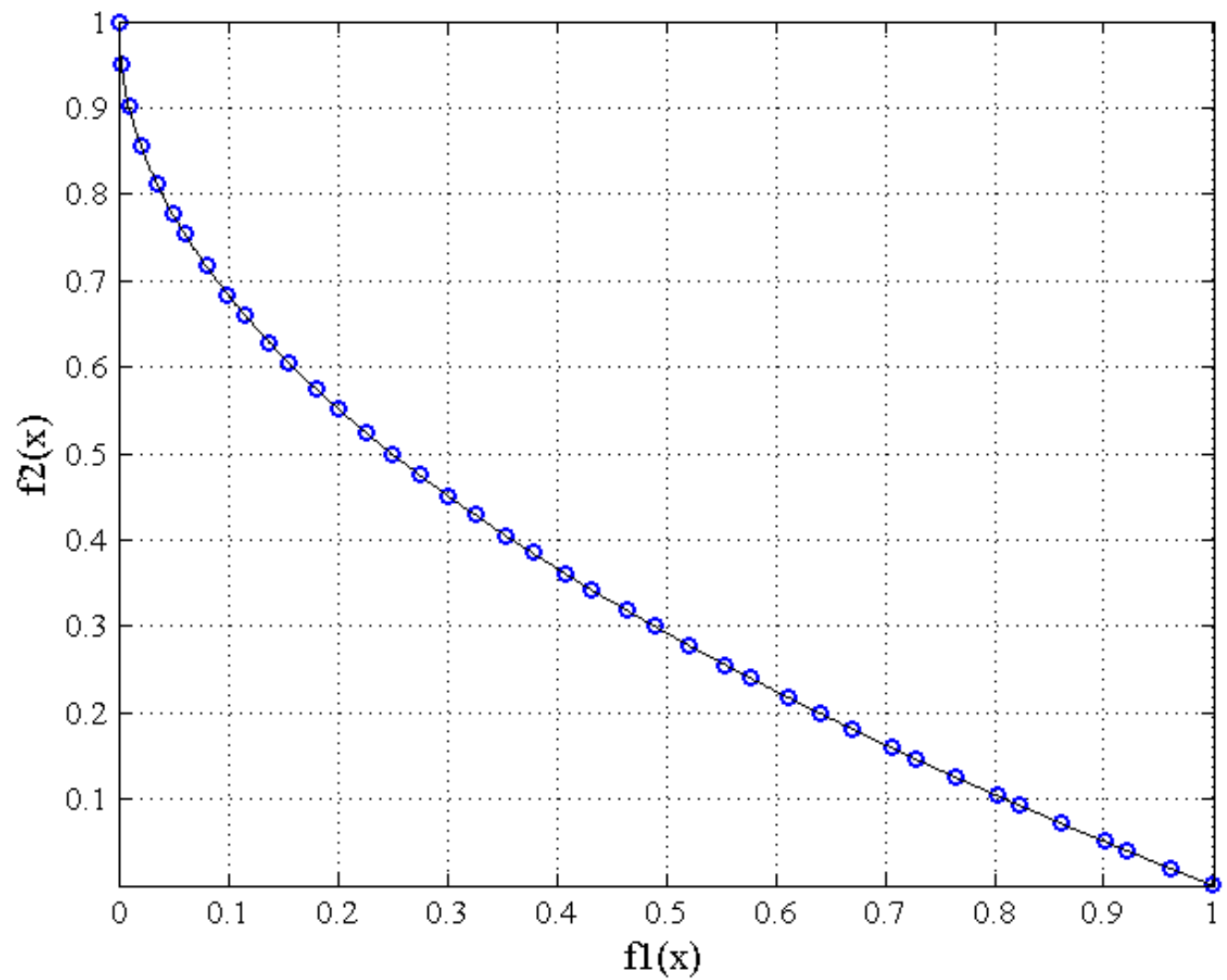

Figure 3.15. ZDT4 Test Function Verification. This plot is the Pareto-frontier for the ZDT4 benchmark test function found in [15]. The blue circles represent the optimal solutions found by the algorithm, and the black line represents the exact analytical solution.

Figure 3.15 is an example of one of the benchmark test functions, specifically ZDT4, used to test the speed and accuracy of the algorithm used in this study. The algorithm performed extremely well with the optimal solutions produced by the algorithm matching the analytical solution within roughly 20,000 function evaluations. This performance is on par with similar genetic algorithms this algorithm is based on. 


\subsection{Implementation in MATLAB and STK}

The algorithm described in section 3.4.2 is written in MATLAB and is the primary tool used to obtain the Pareto-frontiers for this study. Systems Tool Kit (STK) is utilized in this study to perform numerical simulations of the satellite constellations and obtain the necessary objective fitness data. An interface between MATLAB and STK is established in MATLAB in order to send and receive data relating to the objective functions. The phenotypic information about each satellite in the constellation is sent via this interface to STK, where a numerical simulation in STK will determine the objective fitness data, which will be returned to MATLAB via the interface. There are two different connections types for MATLAB/STK, the COM and TCP/IP connections. The MATLAB/STK interface used in this study is the COM connection, with both connect and object model commands. A very helpful resource for using the MATLAB/STK interface along with a detail list of commands is given by AGI in the STK Programming Interface 10.1.1 [4]. The possible connections between MATLAB and STK are visualized in Fig. 3.16, from [3]. 


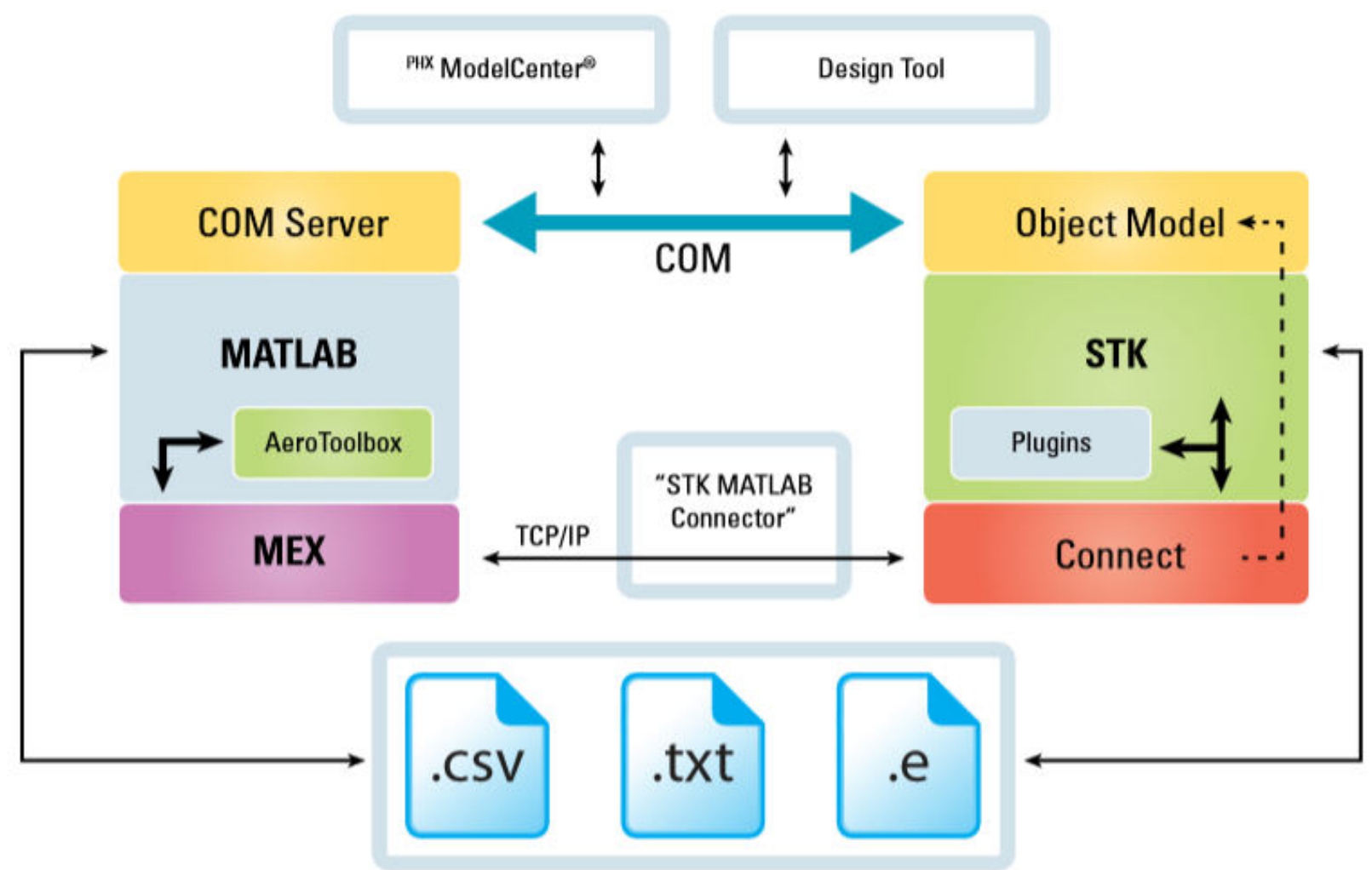

Figure 3.16. MATLAB/STK Interface Schematic. This diagram from [3], depicts the different ways in which data can be sent and received in MATLAB from STK.

In addition to providing the objective fitness data through numerical simulation, STK provides a powerful tool for visualizing satellite constellation systems. The obtained Paretooptimal solutions may be visualized in STK in order to better understand and visualize the phenotypic traits of the optimal solutions.

In this study, a scenario in STK is created which contains a coverage grid over a region on the Earth along with satellite objects in space. Many parameters may be specified such as the time span of the scenario, simulation step size, orbital propagation model, orbital elements of the satellites, locations of grid points, access geometry constraints, and many more parameters. Once the scenario is fully specified in MATLAB through the interface to STK, the physics 
engine will propagate the orbits of the satellites and compute access to the coverage grid points for the length of the simulation. Once the numerical simulation takes place, statistical coverage data may be reviewed in the Coverage Report and Graph Manager in STK. This data may also be requested and accessed in MATLAB via the interface. Several visualizations of an example scenario containing a global coverage grid with a 60:5/5/1 Walker constellation are provided in Fig. 3.17 and 3.18.

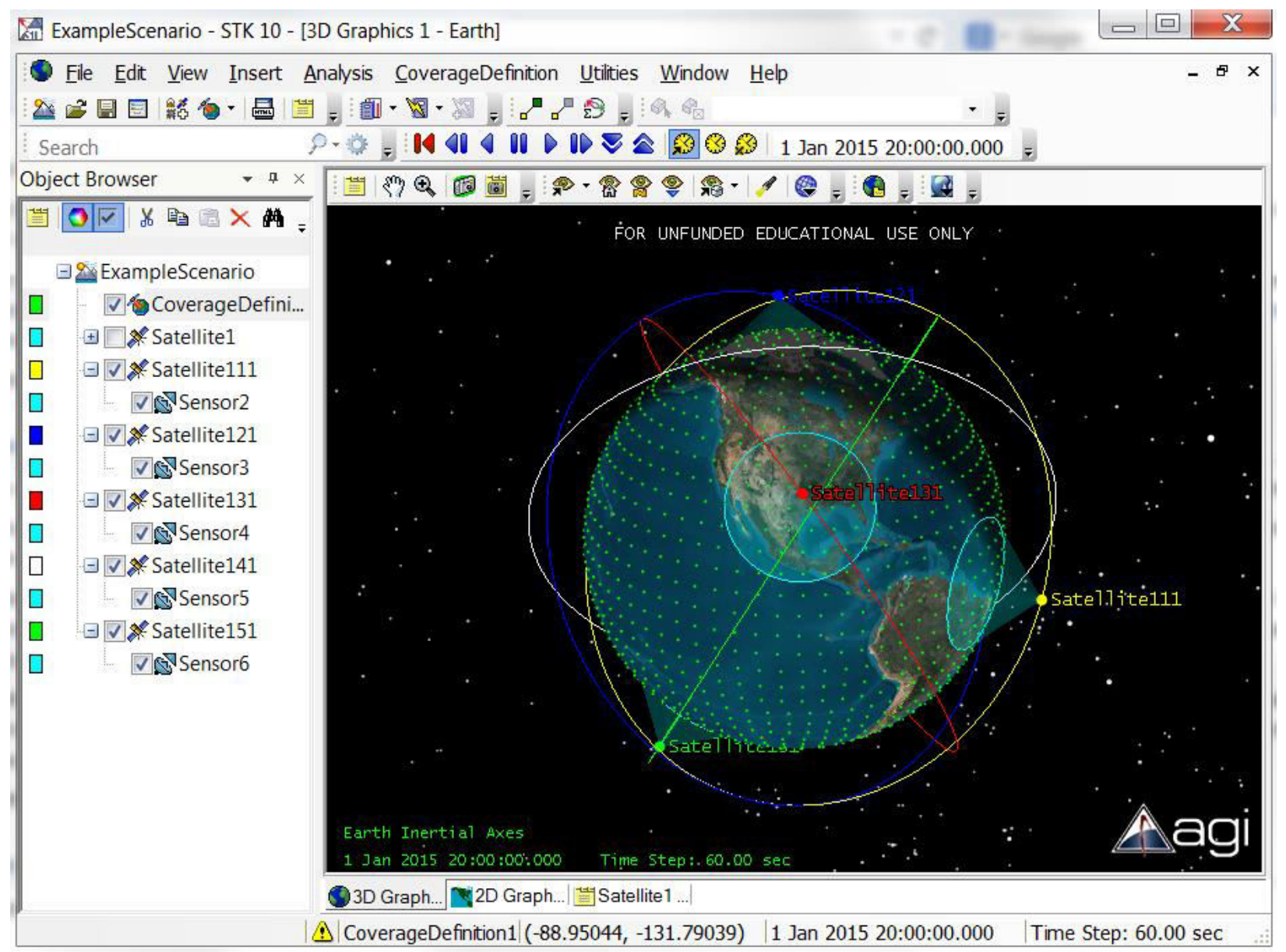

Figure 3.17. STK Walker Constellation 3D Visualization. This image depicts the STK GUI for a 60:5/5/1 Walker constellation and a global coverage grid. 


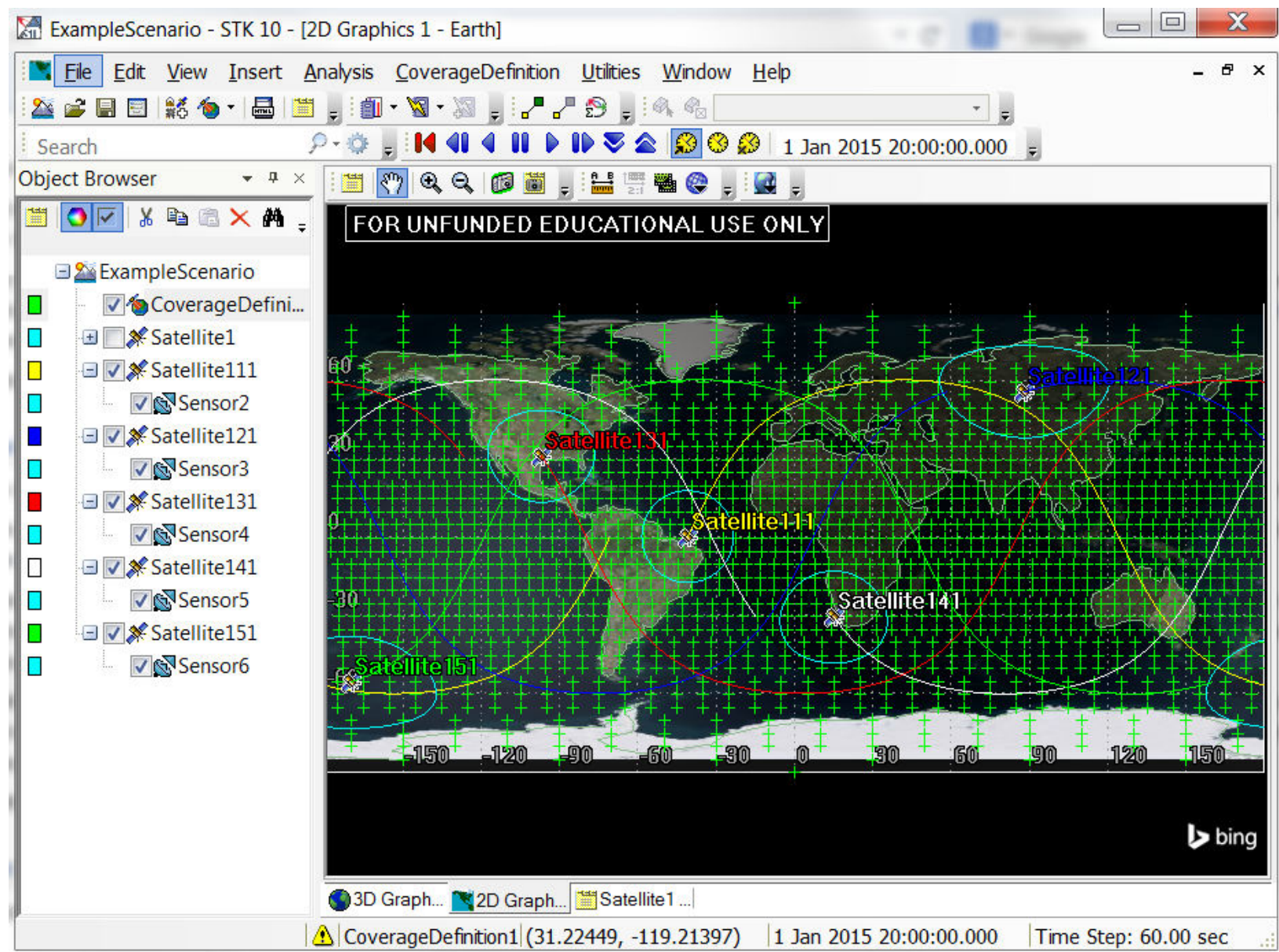

Figure 3.18. STK Walker Constellation 2D Ground Track Visualization. This image depicts the STK GUI for a 60:5/5/1 Walker constellation and a global coverage grid.

STK and the MATLAB/STK interface is a very powerful tool for providing numerical simulation coverage data, especially for a constellation system. The ease in changing parameters and the ability to create nearly every imaginable system scenario makes STK ideal for this application. 


\section{RESULTS}

This chapter presents the results of this study, broken into several case studies, which include the obtained Pareto-frontiers along with a detailed analysis of the observed trends. In addition to an analysis of the objective space, the optimal design space will be characterized.

It should be noted that the Pareto-frontier plots presented in this study are approximated frontiers, as true Pareto-optimal frontiers would require an enumerative method to ensure Paretooptimality over the entire search space. Although the Pareto-frontiers are approximated, they have still be developed over thousands of function evaluations and the trends they exhibit are worth discussing. Even though they may not be truly Pareto-optimal over the entire search domain, they are still solutions that exist within that domain. As such the obtained Paretofrontiers may be treated as solutions where the true optimal solutions are at least this good if not better.

\subsection{Case 1}

The main goal of performing this case study is to characterize the design and objective space surrounding small LEO regional imaging constellation systems. Another goal is to see how the latitude of a target region has an impact on the performance of a small LEO constellation system providing coverage to that region. In this study, the Walker constellation design will be used to optimize the small LEO regional constellation system at a range of latitude target points. The objectives will include minimizing the number of satellites, minimizing the altitude of the constellation, maximizing the minimum daily visibility time, and minimizing the maximum revisit time. A discussion of the setup and parameters used in the Case 1 study will be 
presented in Section 4.1.1. The results of Case 1 will then be presented and discussed in Section 4.1.2.

\subsubsection{Case 1 Problem Formulation}

In order to see how the latitude of a small target region affects the performance of a regional constellation system, five different targets at varying latitudes of $0,22.5,45,67.5$, and 90 degrees will be used. These targets were selected as they provide an even spread over the entire latitude range. Since the dynamics of the satellite motion rely only on the two-body EOM coupled with the $\mathrm{J} 2$ perturbation, there exists total symmetry on either side of the equator in latitude, as well as total symmetry in longitude. Thus, a target region at 45 degrees versus -45 degrees latitude will exhibit the same performance over time for a circular constellation. If an elliptical constellation is utilized, the achievable performance over time will be identical for two targets at 45 and -45 degrees latitude, as long as the argument of perigee is rotated by 180 degrees. Additionally, a target region at a specified latitude will show the exact same achievable performance at any given longitude. For this reason, the target regions are spread evenly between 0 and 90 degrees in latitude and are located at an arbitrarily selected longitude of 0 degrees.

A minimum ground elevation angle of 60 degrees is utilized throughout this case study. For a remote sensing satellite, the minimum ground elevation angle plays a huge role in the quality and type of image that is gathered by the space-based sensor. High elevation angles are often required in order to gather usable data. According to [30], the typical minimum ground elevation angle for a remote sensing system is 60 degrees. For the purpose of this study, a 60 degree minimum ground elevation angle is used as it provides a high enough angle for good data, 
while not too high so that revisit times are in excess. Note that a minimum ground elevation angle of 0 degrees, which provides the maximum in pure geometrical access, would provide much better constellation performance at the cost of poor image quality.

In order to reduce computational complexity due to the fact that so many simulations must be run for varying latitudes, the target region is represented by a single grid point. This grid point can easily represent a target city, and the trends that will be analyzed in the Paretofrontiers can be extrapolated to small regions, perhaps the size of a small country.

The measure of constellation performance will be based on maximizing the minimum daily visibility time (MDVT) and minimizing the maximum revisit time (MRT). The MDVT is simply the smallest amount of total time in a single day that coverage will be provided. The MRT is simply the largest gap in coverage that will occur. These two performance metrics are used because they provide a very good indication of the worst case performance, specifically in the amount of access that will be provided along with the size of the coverage gaps. In addition, the altitude of the constellation and the number of satellites are used to help quantify performance. Constellation at lower altitude will provide better resolution images, and hence increased performance. Additionally, fewer satellites will result in decreased cost of the overall system.

To characterize this type of problem, several restrictions will be placed on the orbital elements of the spacecraft in the constellation. The constellation design space will be limited to the Walker Delta Pattern. Walker-delta patterns are widely used patterns that result in very symmetrical constellation designs. This symmetry allows the constellation to maintain the same geometry over long periods of time, in addition to providing very good coverage. Figure 4.1 
shows the structure of the chromosome along with the resulting makeup of the orbital elements for each satellite in the constellation. Table 4.1 shows the boundaries of the design search space.

$$
\text { Chromosome Structure }=\left[\begin{array}{lllllll}
a & \text { inc } & \Omega_{\text {seed }} & \theta_{\text {seed }} & T & P & F
\end{array}\right]
$$

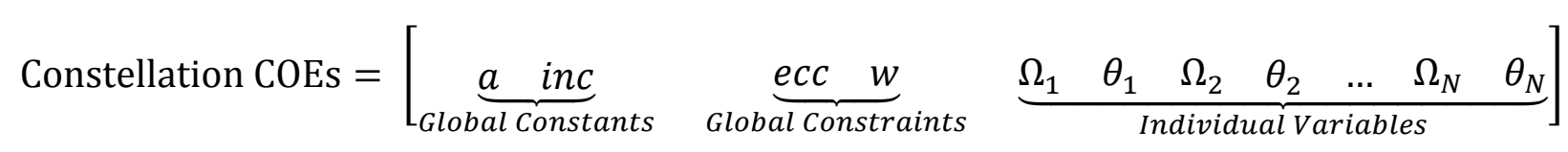

Figure 4.1. Case 1 Chromosome Structure and Resulting Makeup of Constellation COEs. Here the variables to be optimized are represented in the chromosome structure. These variable result in corresponding orbital elements for each satellite in the constellation, which is shown as the constellation COEs.

Table 4.1. Case 1 Design Search Space.

\begin{tabular}{lccc}
\hline \hline Variable & Search Space & Value & Units \\
\hline \hline a & Bounded & $6578-7878$ & $\mathrm{~km}$ \\
ecc & Constant & 0 & - \\
inc & Bounded & $0-90$ & $\mathrm{deg}$ \\
$\mathrm{W}$ & Constant & 0 & $\mathrm{deg}$ \\
$\Omega_{\text {seed }}$ & Bounded & $0-360$ & $\mathrm{deg}$ \\
$\theta_{\text {seed }}$ & Bounded & $0-360$ & $\mathrm{deg}$ \\
$\mathrm{T}$ & Bounded & $1-6$ & - \\
$\mathrm{P}$ & Bounded & Factors of T (1-6) & - \\
$\mathrm{F}$ & Bounded & $0-(\mathrm{P}-1)$ & -
\end{tabular}

It is shown that the decision space has several bounds and constraints in order to simplify and characterize the problem. The design space is limited strictly to Walker-delta patterns, resulting in all of the satellites having the same altitude, inclination, eccentricity, and argument of perigee. The altitude of the constellation is bounded between $200 \mathrm{~km}$ and $1500 \mathrm{~km}$. Almost all spacecraft in LEO and constellation systems are below $1500 \mathrm{~km}$ in altitude, and the search will therefore take place within this range. The inclination of the constellation is bounded between 0 and 90 degrees. Although you can get slightly better revisit times with retrograde orbits, it is costly to place spacecraft into retrograde orbits. Nearly all spacecraft are prograde with the 
exception of sun-synchronous spacecraft, with inclinations just above 90 degrees. The eccentricity of the constellation is constant at 0 ; only circular orbits will be studied. It is possible to get better performance with eccentric orbits, but adding eccentricity into the constellation can greatly complicate the system. Since the altitude is bounded between 200 and $1500 \mathrm{~km}$, the maximum eccentricity available would be approximately 0.09 . The performance would be minimally affected by introducing this slight eccentricity, and several unwanted effects would occur. If the perigee point was at $200 \mathrm{~km}$ and apogee was at $1500 \mathrm{~km}$, the variation in drag over the entire orbit would cause unwanted oscillations and variations in the mean orbital elements that would affect the geometry of the constellation over time. This study will therefore be restricted to circular orbits in order to reduce these unwanted effects. Due to this restriction to circular orbits, the argument of perigee and the mean anomaly provide the same information, and therefore only one of these elements is needed. The argument of perigee will therefore be kept constant at 0 and the mean anomaly will be allowed to vary between 0 and 360 degrees. The orbital elements of a seed satellite will be optimized in the design process, with the other satellites built around the seed satellite in a Walker-delta pattern. The other variables that need to be optimized are thus the number of spacecraft, $\mathrm{T}$, the number of orbital planes, $\mathrm{P}$, and the relative spacing between satellites in adjacent planes, F. The number of spacecraft is bounded in this study between 1 and 6 satellites. Since the region of interest is a single target point, it can be assumed that mission designers would want to launch a minimal number of spacecraft to provide this type of coverage. In addition, placing the bounds between 1 and 6 spacecraft reduces the computational cost and allows a more detailed analysis on a more specific problem. Although one satellite is not a constellation, coverage for a single satellite is included in this study as a baseline to compare performance between a single satellite and larger constellation systems. The 
number of orbital planes is simply bounded based on the number of satellites in the constellation as defined by the Walker-delta pattern. Similarly, the relative spacing between satellites in adjacent planes is bounded based on the allowable Walker-delta pattern values. The chromosome is made up of the seven values shown in Figure 4.1. These seven variables will be optimized and can be mapped to the individual COEs of each satellite in the constellation as also shown in Fig. 4.1. The problem is therefore defined as optimizing coverage for small, circular, common inclination, regional, LEO, Walker constellations.

The propagation of the satellites will be performed using the J2Perturbation orbit propagator in STK. The J2Perturbation orbit propagator was chosen because it represents the largest perturbative force in this problem. Since it causes such a large change over time in the ascending node, it cannot be neglected and must be used in this study. The other perturbations are either too small to be considered, or can be countered to maintain a constant constellation geometry with relatively small station keeping maneuvers. A minimum propagation step size, as well the minimum access computation step size will be set to 60 seconds. The propagator will automatically determine the best step size at each point in the propagation, without going below 60 seconds. An informal study was conducted that determined for this altitude range and for the ground elevation angles used in this study, the trends in the Pareto-frontiers were not noticeably affected when the step size was reduced below 60 seconds. The simulation time span is given as 30 days. Due to the fact that the altitude is restricted to a maximum of $1500 \mathrm{~km}$, the orbital period of the spacecraft is relatively small and the spacecraft in the constellation will orbit the Earth many times each day. A time span of several days is shown to be produce consistent results, however a time span of 30 days is used to ensure consistent and accurate statistical 
results. A time span longer 30 days shows no noticeable affect on the trends of the Paretofrontier. Table 4.2 summarizes the parameters used in the STK simulation.

Table 4.2. STK Simulation Parameters.

\begin{tabular}{lcc}
\hline \hline \multicolumn{1}{c}{ Parameter } & Value & Units \\
\hline \hline Propagator Type & J2Perturbation & - \\
Propagation Step Size & 60 & $\mathrm{sec}$ \\
Access Computation Step Size & 60 & $\mathrm{sec}$ \\
Simulation Time Span & 30 & days
\end{tabular}

The constellation performance metric, minimum daily visibility time will be obtained by a numerical simulation in STK. The MDVT will be determined by breaking the simulation run into 30, 24-hour time segments. The amount of time the target region is visible to the constellation during each 24-hour time segment is recorded as the DVT. The MDVT is thus the maximum DVT of all 30 segments. The constellation performance metric, maximum revisit time (MRT), will also be obtained by a numerical simulation in STK. The MRT will be determined by simply obtaining the longest period with no access to the ground point (largest gap in coverage) over the entire simulation run.

The $\varepsilon N S G A-I I$ algorithm used in this study has several tuning parameters that are defined in Table 4.3.

Table 4.3. \&NSGA-II Tuning Parameters. Parameter

\begin{tabular}{lc}
\hline \hline Initial Population Size & 5 \\
Crossover Probability & 1 \\
Mutation Probability ( $\mathrm{N}_{\text {genes }}$ is the numer of genes) & $1 / \mathrm{N}_{\text {genes }}$ \\
Whole Arithmetic Recombination $\alpha$ bounds & {$[-.25,1.25]$} \\
Gaussian Mutation Standard Deviation & 0.2
\end{tabular}


Table 4.4 shows the design objectives along with the corresponding epsilon grid values. The range of the MDVT is simply bounded between 0 and 1440 minutes due to the number of minutes in a given day. Additionally, the MRT has no set range; although due to a simulation time span of 30 days the largest MRT possible is 30 days. An MDVT of 1440 minutes and MRT of 0 minutes would mean there is $100 \%$ coverage over the entire simulation run. The epsilon values shown in Table 4.4 were chosen as they allow good visualization of the Pareto-frontiers without resulting in too many points to be computationally expensive. The main limiting epsilon grid dimension is the constellation altitude at $25 \mathrm{~km}$.

Table 4.4. Case 1 Design Objectives and Corresponding Epsilon Grid Values.

\begin{tabular}{lcccc}
\hline \hline \multicolumn{1}{c}{ Design Objective } & $\begin{array}{c}\text { Optimization } \\
\text { Direction }\end{array}$ & Range & Epsilon Value & Units \\
\hline \hline Number of Spacecraft & Minimize & $1-6$ & 1 & - \\
Constellation Altitude & Minimize & $200-1500$ & 25 & $\mathrm{~km}$ \\
Min Daily Visibility Time & Maximize & $0-1440$ & 1 & min \\
Max Revisit Time & Minimize & - & 1 & min
\end{tabular}

\subsubsection{Case 1 Results}

First, several hypotheses will be presented followed by the actual results. It is expected that the best revisit times and daily visibility times will be for a target region located at the poles. This is due to the fact that the target region will remain stationary with respect to the constellation. Additionally, the worst achievable revisit times and daily visibility times should be for a target region directly in between the poles and the equator, at 45 degrees latitude. High altitudes should provide better daily visibility times and worse revisit times, similarly low altitudes should provide better revisit times but worst daily visibility times. At lower altitudes, the satellite will have a smaller orbital period and will thus be able to visit the target site more 
frequently, but will not spend as much time over the target site. Increasing the number of satellites should also result in better revisit times and daily visibility times, although by how much is unknown. The optimal inclination should be similar to the latitude of the target region, and the optimal number of orbital planes is unknown. Finally, some discontinuity in the Paretofrontier should exist due to the fact that symmetric Walker constellation patterns are being used.

The obtained Pareto-frontiers will now be displayed. The first set of plots shown, Fig. 4.2-4.6, will be Pareto hypervolumes, which provide a visualization of the four-dimensional objective space. Note that each point in the plot represents an entire constellation design that is characterized by a given number of satellites, each with its own unique orbital elements. In order to obtain these plots, the algorithm was run over a period of several hours to a full day, with the number of function evaluations ranging between 30,000 and 100,000. Each plot depicts the four design objectives, along with arrows indicating the optimization direction. Five plots are therefore shown, Fig. 4.2-4.6, for each latitude region (0, 22.5, 45, 67.5, and 90 degrees), and are shown in order of increasing latitude. Note that the scale of the axis for MDVT and MRT are different for all five plots. 


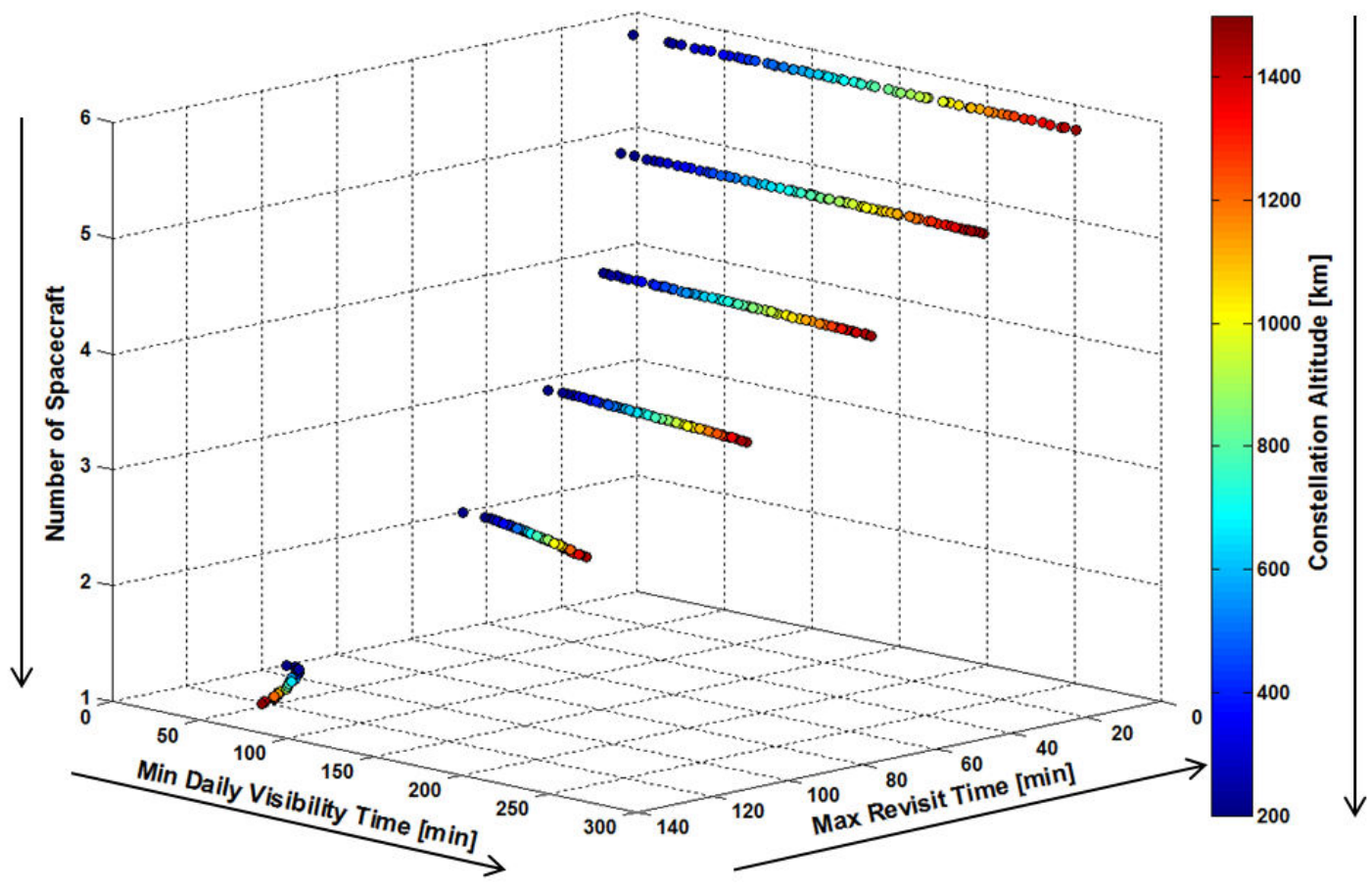

Figure 4.2. Number of Spacecraft vs. Constellation Altitude vs. MDVT vs. MRT for 0 Degree Latitude Target. This plot shows the four-dimensional Pareto-hypervolume, with arrows indicating the optimization direction.

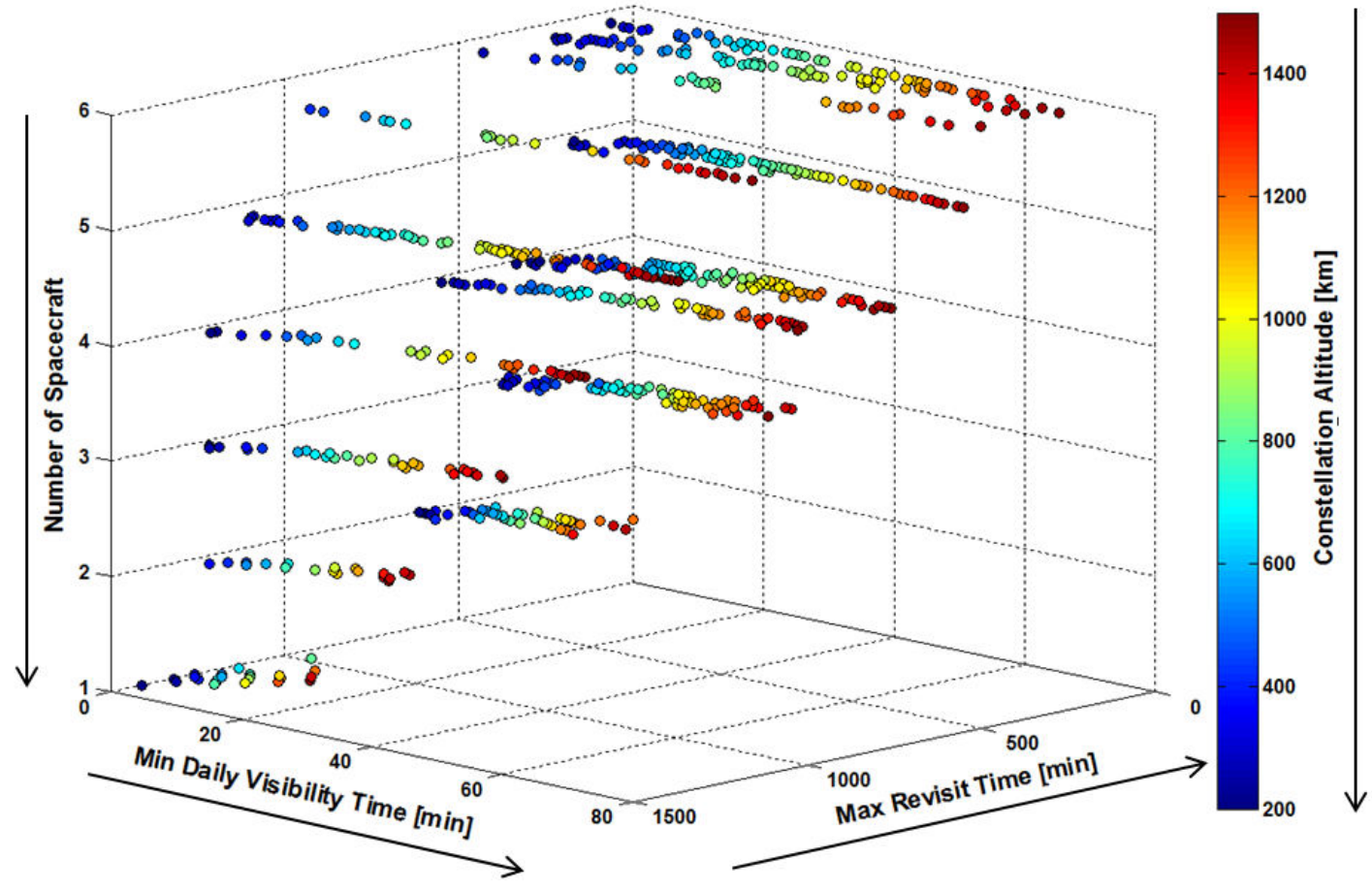

Figure 4.3. Number of Spacecraft vs. Constellation Altitude vs. MDVT vs. MRT for 22.5 Degree Latitude Target. This plot shows the four-dimensional Pareto-hypervolume, with arrows indicating the optimization direction. 


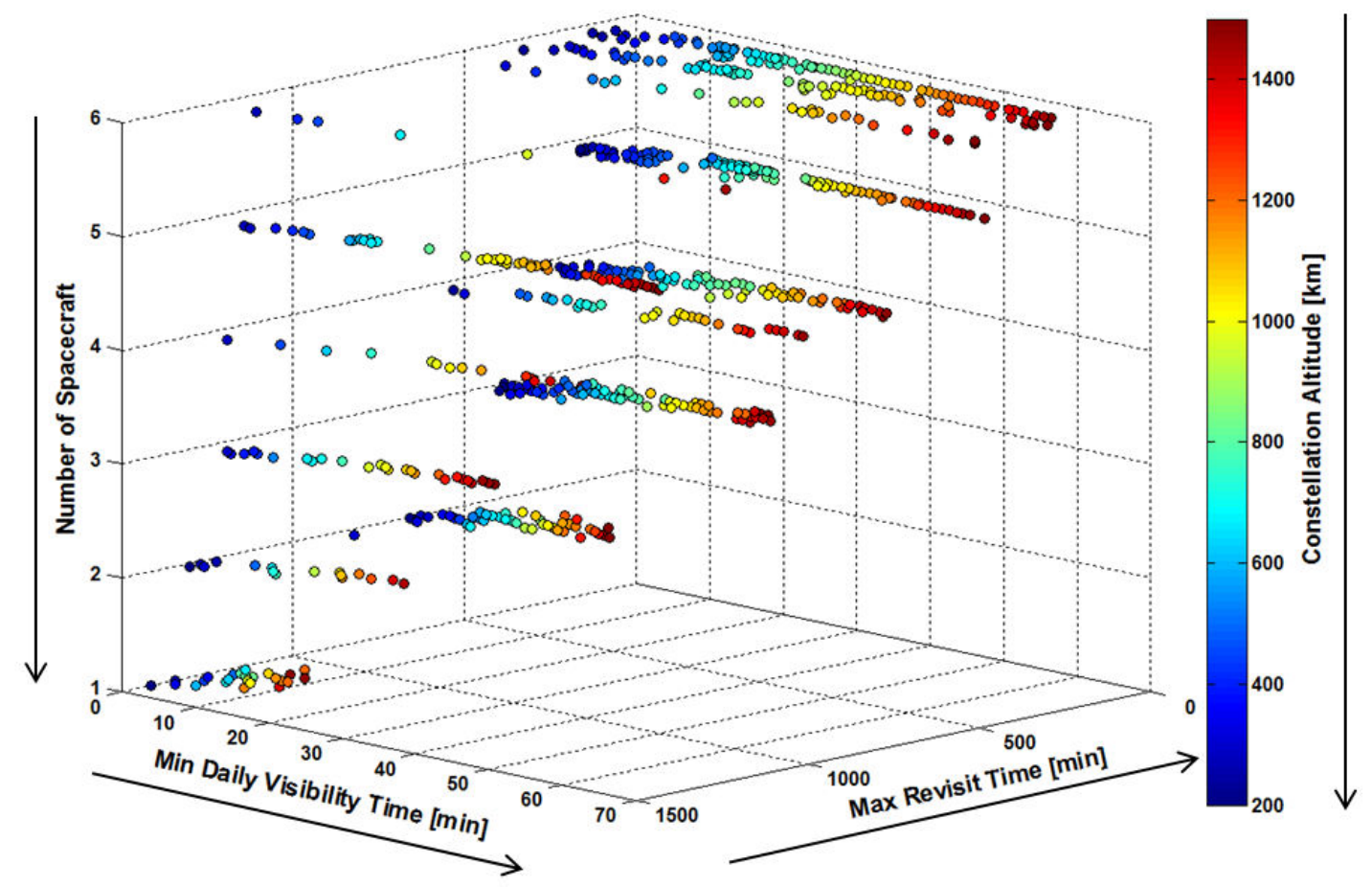

Figure 4.4. Number of Spacecraft vs. Constellation Altitude vs. MDVT vs. MRT for 45 Degree Latitude Target. This plot shows the four-dimensional Pareto-hypervolume, with arrows indicating the optimization direction.

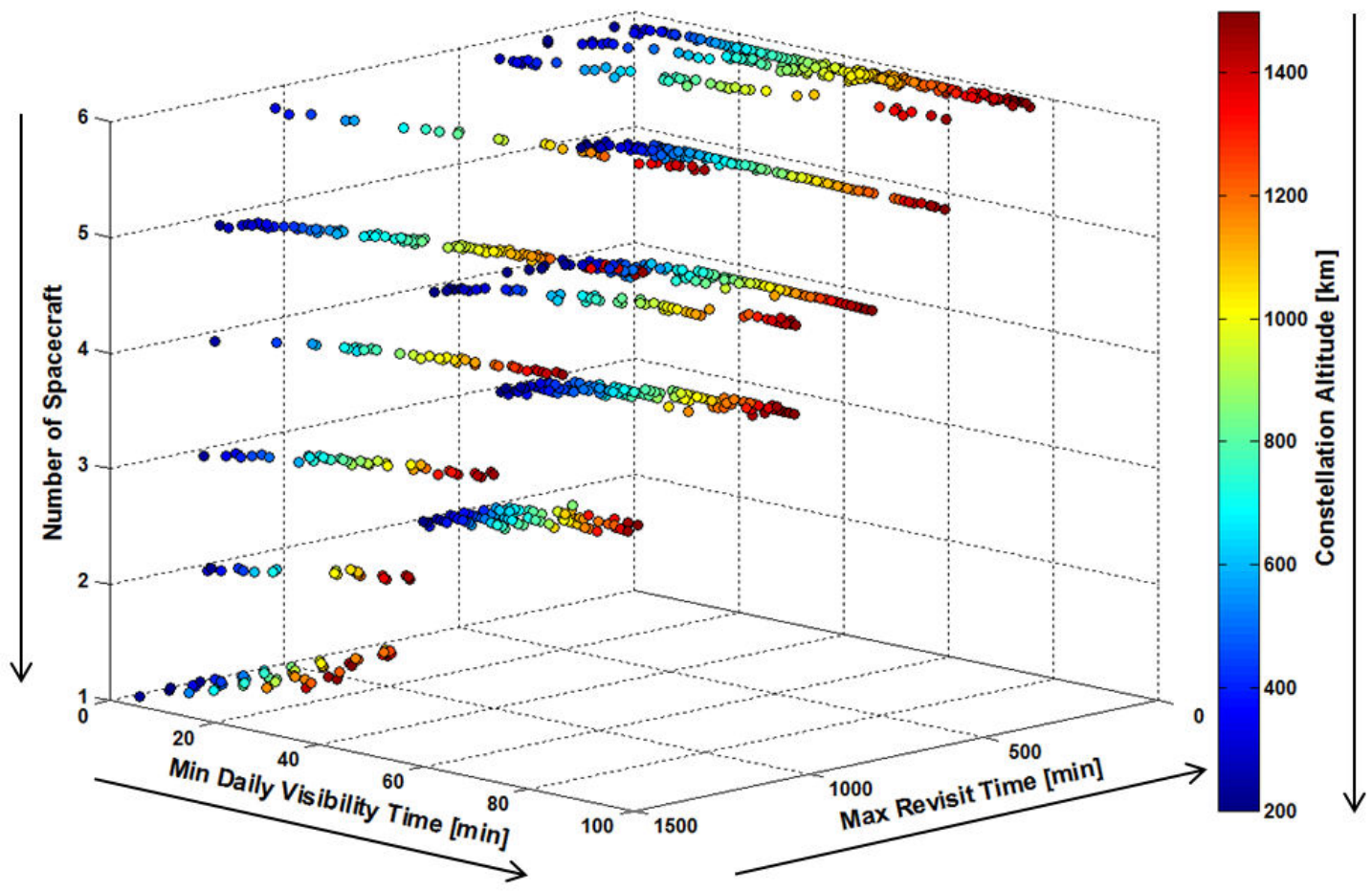

Figure 4.5. Number of Spacecraft vs. Constellation Altitude vs. MDVT vs. MRT for 67.5 Degree Latitude Target. This plot shows the four-dimensional Pareto-hypervolume, with arrows indicating the optimization direction. 


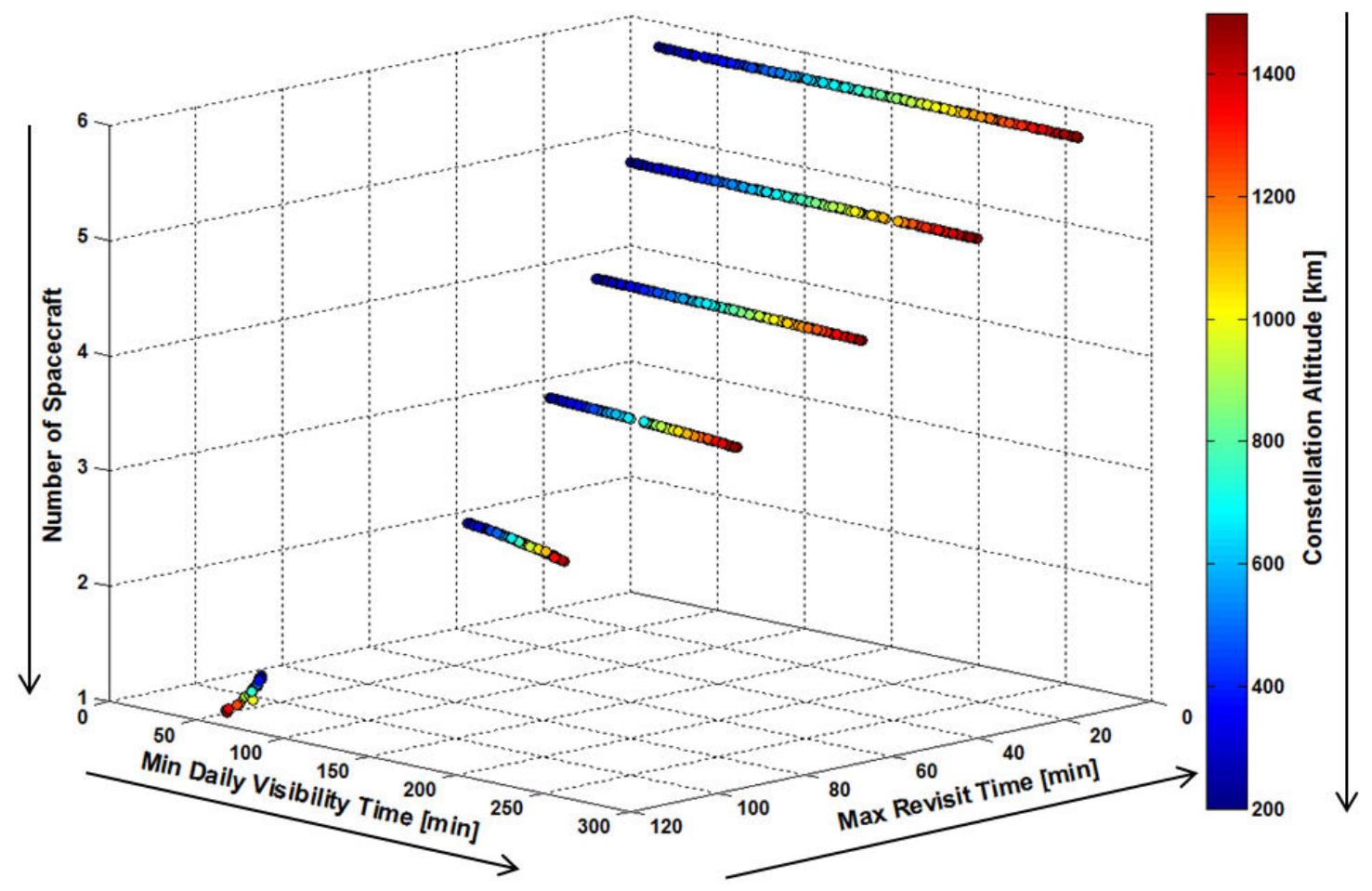

Figure 4.6. Number of Spacecraft vs. Constellation Altitude vs. MDVT vs. MRT for 90 Degree Latitude Target. This plot shows the four-dimensional Pareto-hypervolume, with arrows indicating the optimization direction.

These Pareto-frontiers show several basic trends, which are generalized as follows:

- The Pareto-frontier has both obvious and not obvious discontinuities that exist. There are obvious discontinuities in the number of satellites in the constellation, as only integer numbers may be represented. Non-obvious discontinuities exist for the target regions at latitudes of 22.5, 45, and 67.5 degrees, which will be discussed in more detail later.

- As expected, as the number of satellites in the constellation increases the minimum daily visibility time increases and the maximum revisit time decreases, resulting in better performance.

- The constellation altitude has a strong correlation with the minimum daily visibility time; as the altitude increases, the minimum daily visibility time also increases. 
- The maximum revisit time correlates most strongly with the number of satellites in the constellation; with more satellites providing lower revisit times.

- It can be deduced that target regions near the equator and near the poles have much better daily visibility times and revisit times that target regions located in between these regions.

- The target regions at 22.5, 45, and 67.5 degrees latitude all have similar performance, with slight differences. The target region at 45 degrees latitude results in the worst achievable performance, followed by the target region at 22.5 degrees, and then the target region at 67.5 degrees.

- The target region at 90 degrees latitude slightly out performs the target region at 0 degrees latitude.

These basic trends will now be discussed in more detail by breaking the Paretohypervolumes into lower dimensional states. In addition to showing fewer dimensions, several optimization parameters will be shown such as the constellation inclination and the number of planes, in order to better characterize the optimal design space.

The next figure, Fig. 4.7, shows the target region at 45 degrees latitude, with the number of orbital planes represented by the colorbar. This plot is identical to Fig. 4.4 except the colorbar, which shows constellation altitude, is simply replaced by the number of orbital planes in the constellation; the constellation altitude is omitted. 


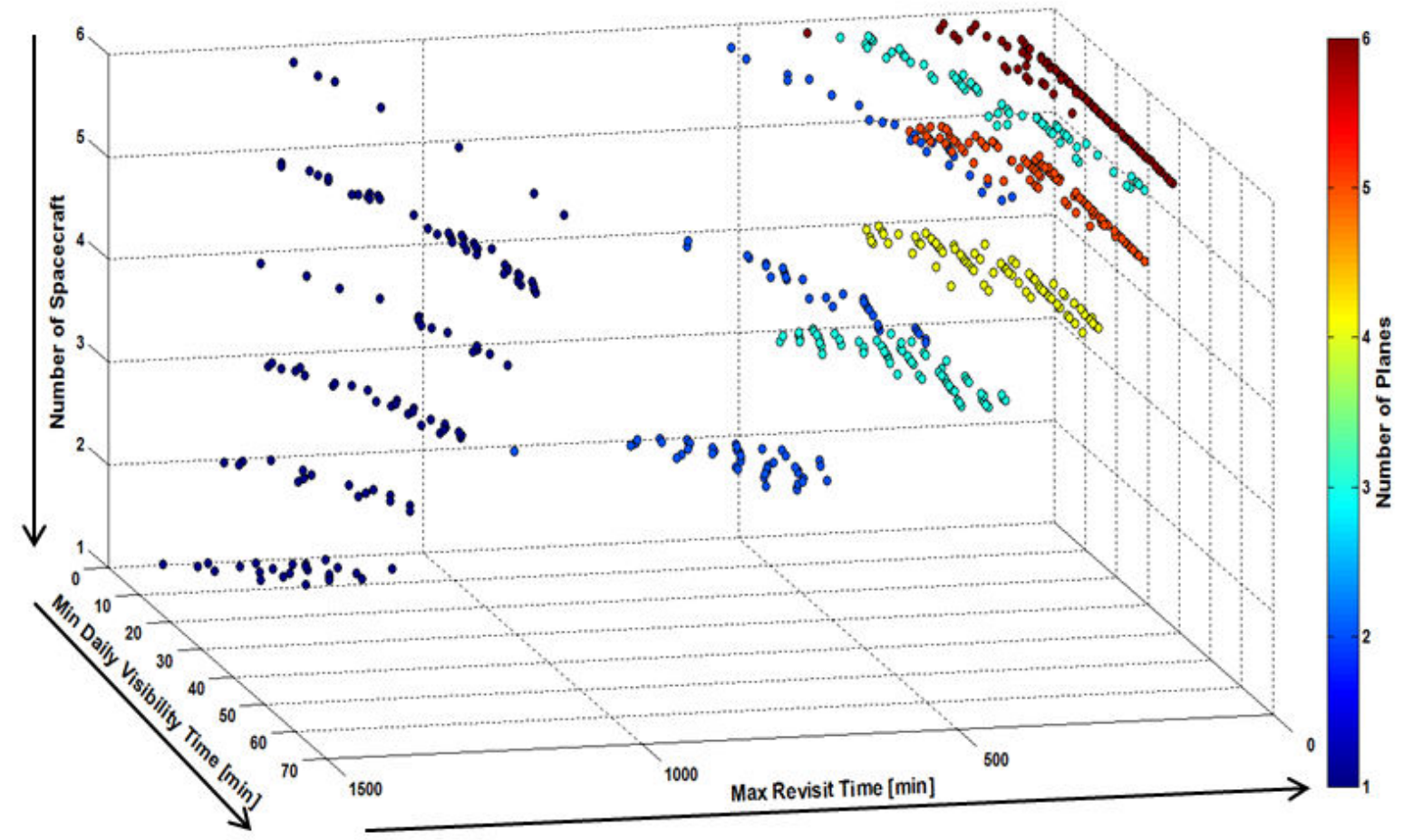

Figure 4.7. Number of Spacecraft vs. MDVT vs. MRT for 45 Degree Latitude Target, with the Number of Planes Shown in Color. This plot shows how the objective space is affected by the number of orbital planes in the constellation, with arrows indicating the optimization direction.

Figure 4.7 shows the objective space affected by the optimization variable, which is the number of planes $(\mathrm{P})$ in a Walker-delta pattern. The objective space for the target regions at $22.5,45$, and 67.5 degrees latitude all show this similar discontinuity. It is evident that as the number of orbital planes increases, the maximum revisit time decreases. It is interesting to note the discontinuities due to the number of orbital planes being constrained to factors of the number of satellites in the constellation. These obvious groupings show six groups of 1-plane constellations, three groups of 2-plane constellations, two groups of 3-plane constellations, and one group of both 4-plane, 5-plane, and 6-plane constellations. It can be concluded from this that if minimizing the revisit times are of interest, it is best to maintain diversity in the number of orbital planes in the constellation. 
The rest of the plots in this section will show the objective space in lower dimensional states in order to better characterize the trends that are present. Figures 4.8-4.12 show specifically how the altitude and number of spacecraft affect the minimum daily visibility time. Note that these plots do not show the MRT objective because it has been omitted to more easily show the trends of interest. Also note that the MDVT axis scale differs between the 0 and 90 degree latitude cases, and the 22.5, 45, and 67.5 degree latitude cases.

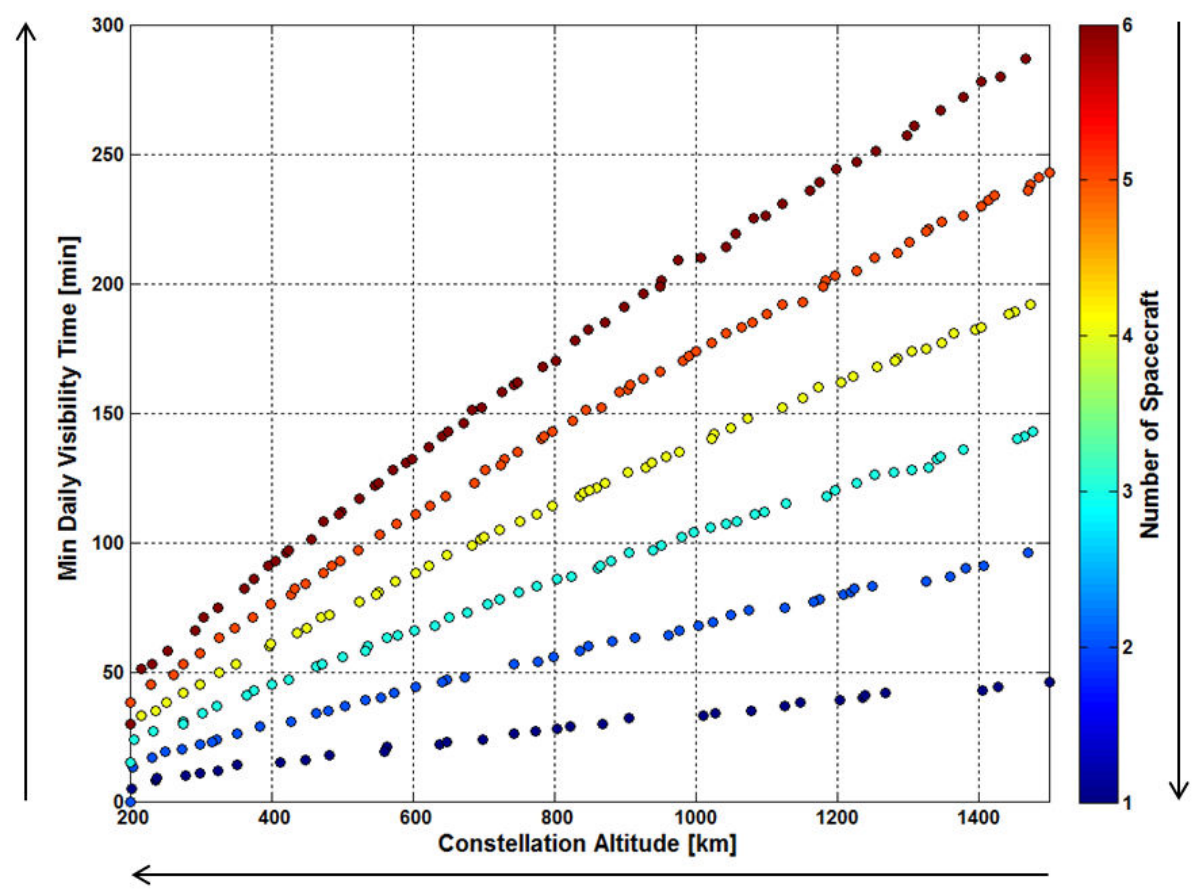

Figure 4.8. Number of Spacecraft vs. MDVT vs. Constellation Altitude for 0 Degree Latitude Target. This plot shows the trends between these three objectives with the MRT omitted. The arrows indicate the optimization direction. 


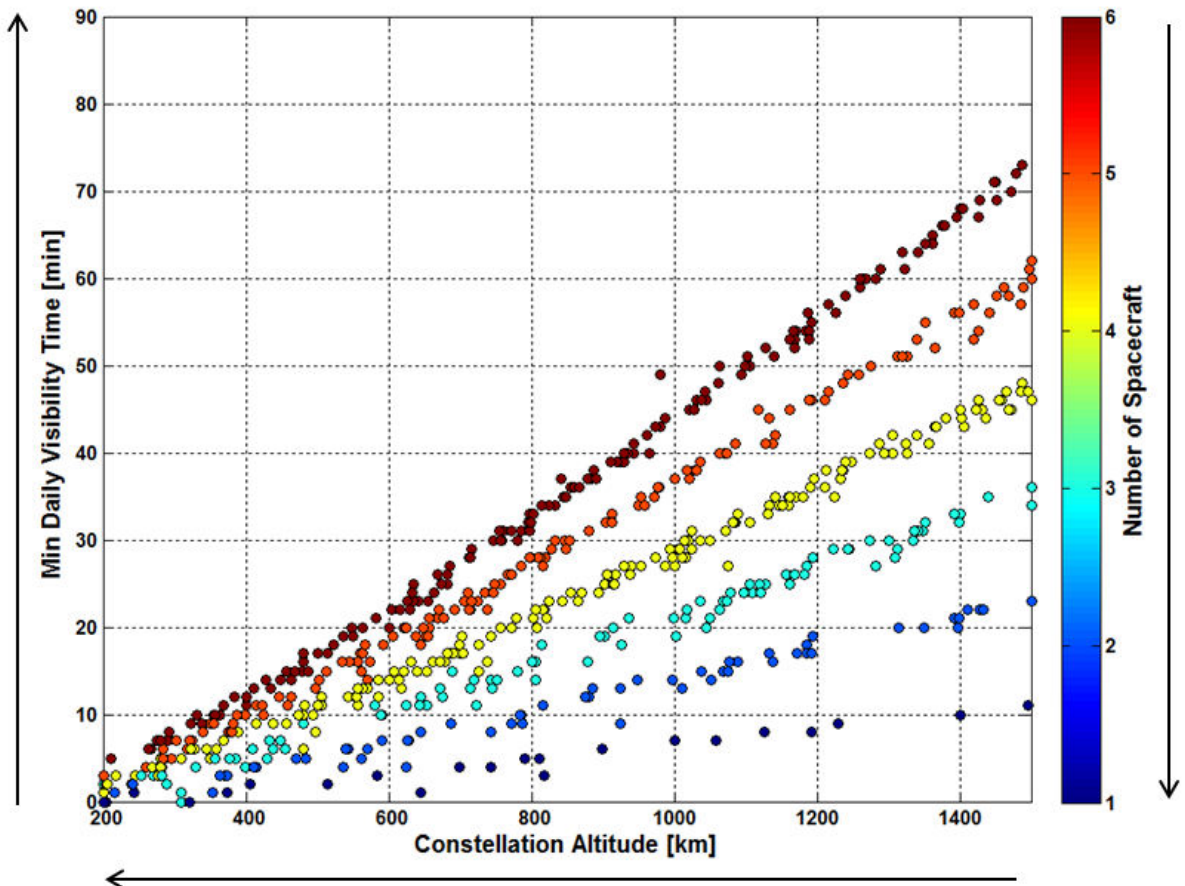

Figure 4.9. Number of Spacecraft vs. MDVT vs. Constellation Altitude for 22.5 Degree Latitude Target. This plot shows the trends between these three objectives with the MRT omitted. The arrows indicate the optimization direction.

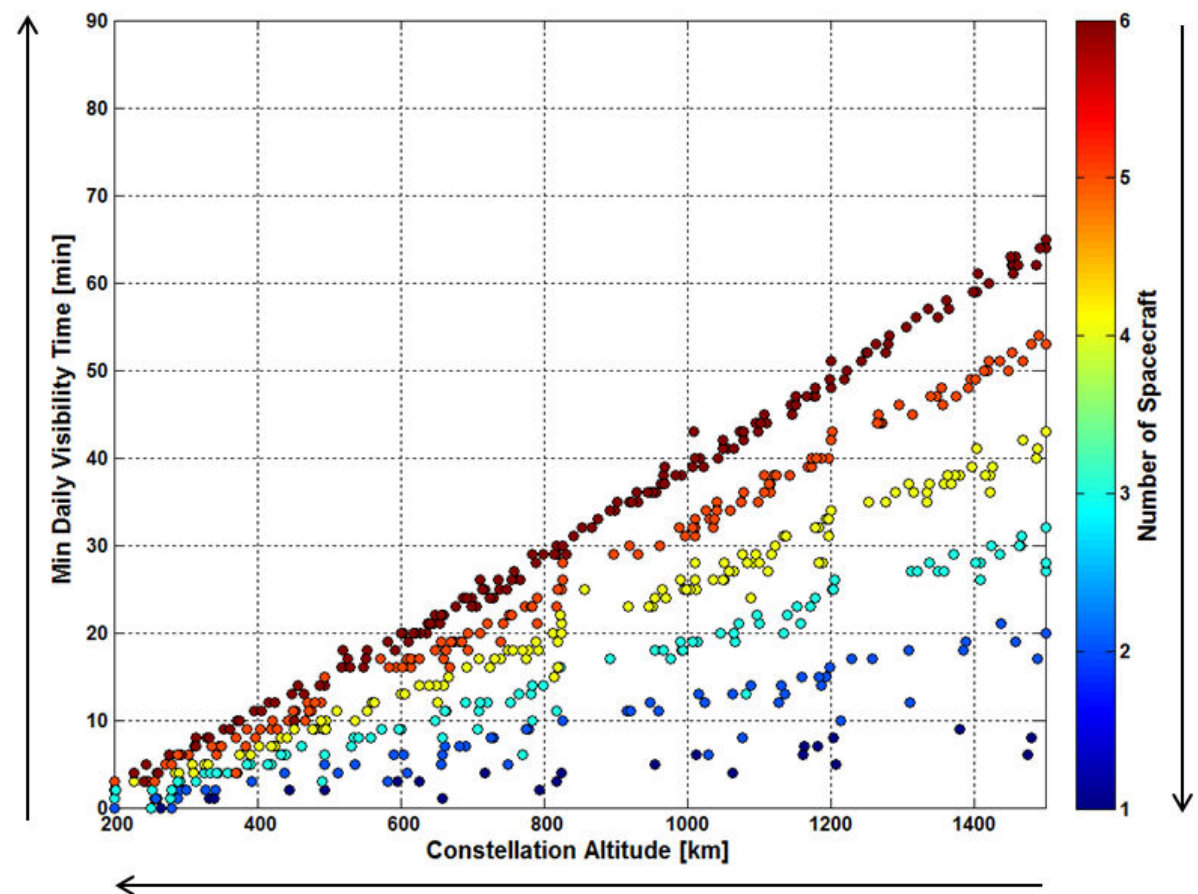

Figure 4.10. Number of Spacecraft vs. MDVT vs. Constellation Altitude for 45 Degree Latitude Target. This plot shows the trends between these three objectives with the MRT omitted. The arrows indicate the optimization direction. 


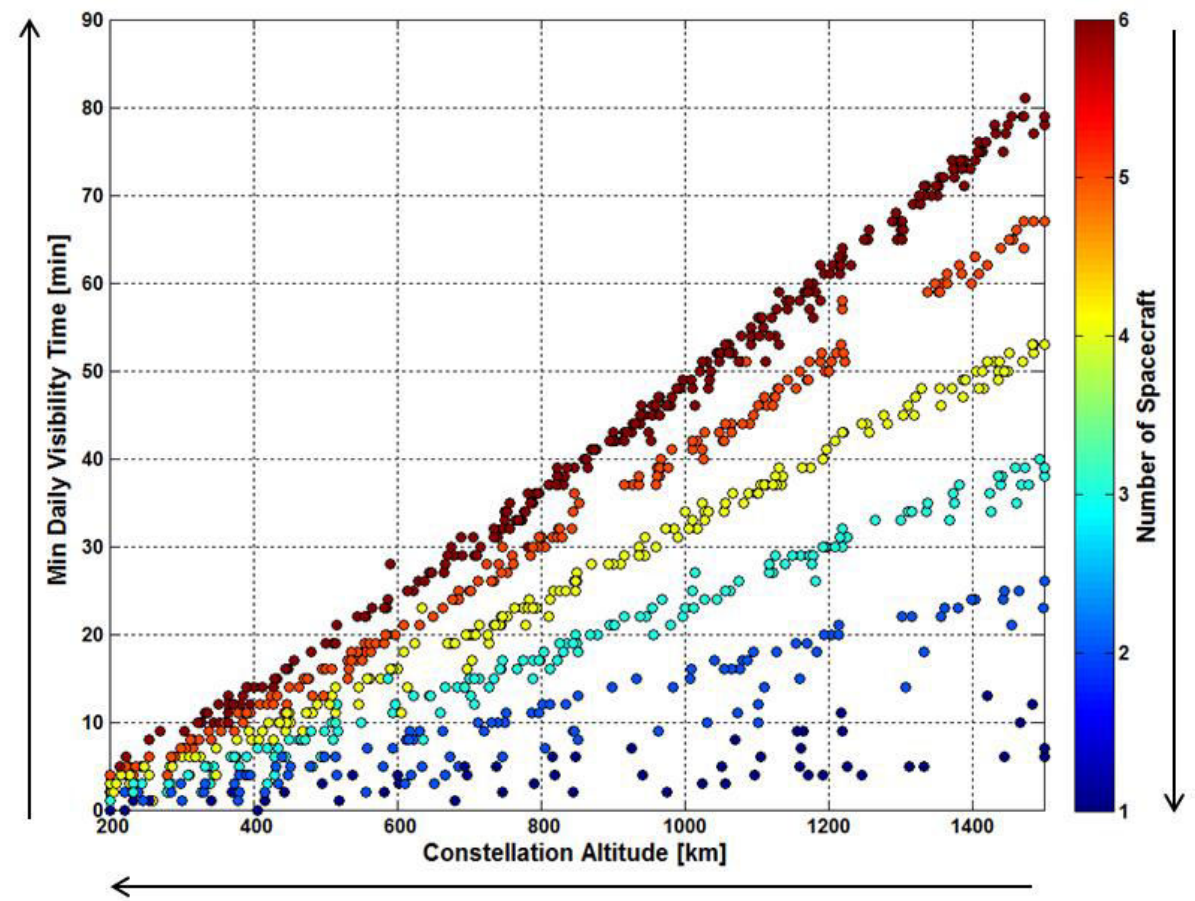

Figure 4.11. Number of Spacecraft vs. MDVT vs. Constellation Altitude for 67.5 Degree Latitude Target. This plot shows the trends between these three objectives with the MRT omitted. The arrows indicate the optimization direction.

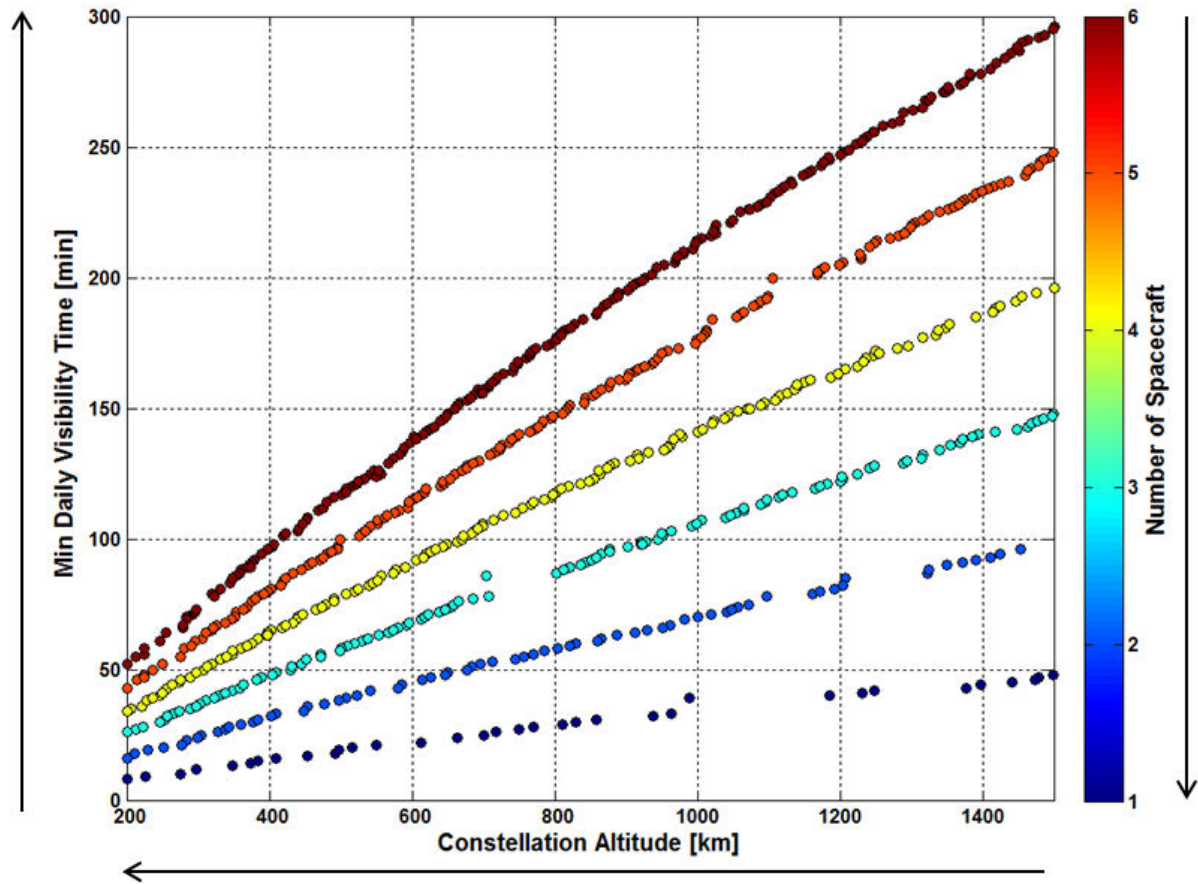

Figure 4.12. Number of Spacecraft vs. MDVT vs. Constellation Altitude for 90 Degree Latitude Target. This plot shows the trends between these three objectives with the MRT omitted. The arrows indicate the optimization direction. 
Several interesting trends emerge by looking at Fig. 4.8-4.12, which show the relationship between the number of spacecraft, minimum daily visibility time, and the altitude of the constellation at varying latitude target regions. These trends are characterized as follows:

- As the altitude of the constellation increases, the MDVT always increases with a near linear relationship. For the target regions at 0 and 90 degrees latitude, this relationship is slightly concave in nature. For the target regions at 22.5, 45, and 67.5 degrees latitude, this relationship is slightly convex in nature.

- The spread in MDVT between 1 and 6 spacecraft is small at low constellation altitudes and is much larger at higher constellation altitudes.

- The relationship between the number of spacecraft and MDVT is also very linear in nature. Increasing the number of spacecraft from 1 to 2 causes the MDVT to increase roughly two-fold. Similarly, increasing the number of spacecraft from 1 to 6 causes the MDVT to increase roughly six-fold.

- For the target regions at 0 and 90 degrees latitude, the achievable MDVT is very similar, with slightly increased performance at 90 degrees latitude.

- The worst achievable performance is for a target region at 45 degrees latitude, followed by 22.5 degrees and 67.5 degrees. These three regions perform very similarly, with the spread in MRT for the six satellite constellation design going from several minutes at low altitudes to nearly 20 minutes at higher altitudes.

The following five plots, Fig. 4.13-4.17, show the same previous five plots, Fig. 4.8-4.12, except the colorbar showing the number of spacecraft is replaced by the constellation inclination. The number of spacecraft may be deduced in these plots by referring to Fig. $4.8-4.12$ or by 
simply noting the six obvious curved groupings. The purpose of showing the inclination here is to see how this variable parameter that is to be optimized in the optimization process affects the performance trends. Note that the axis scale of the colorbar changes between for each of the five plots in order to better visualize the small differences in inclination for each latitude region.

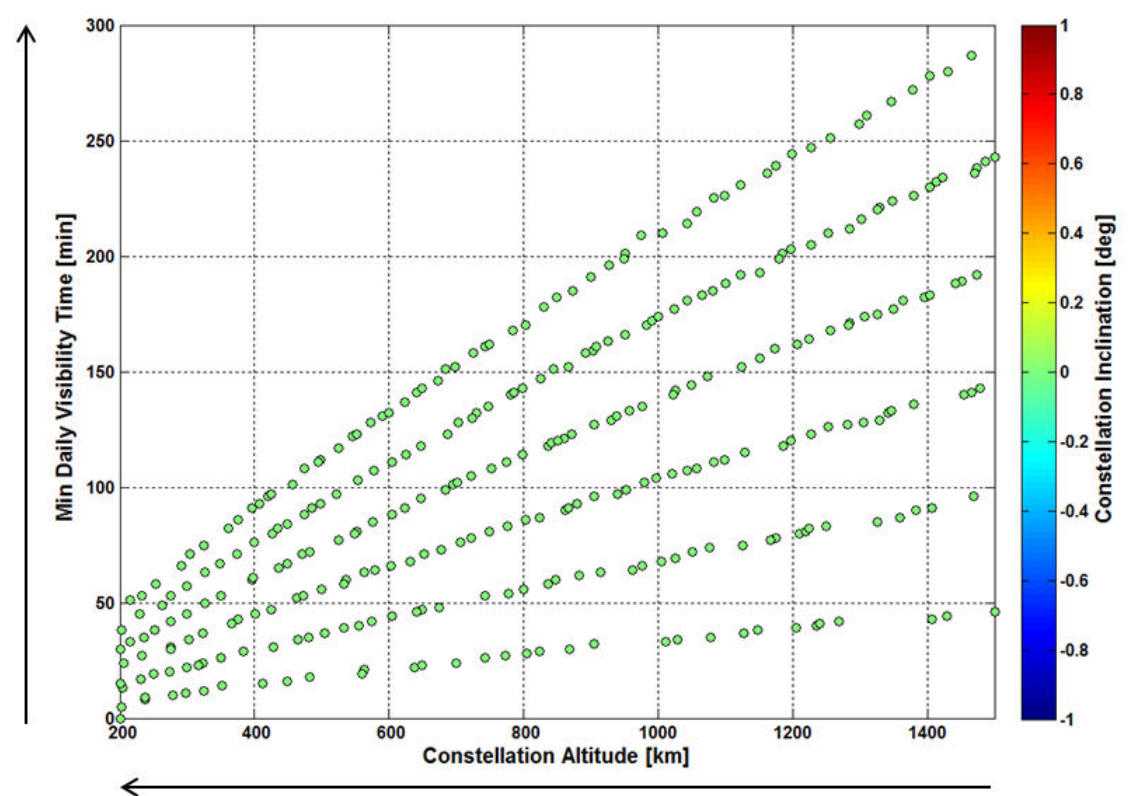

Figure 4.13. Number of Spacecraft vs. MDVT vs. Constellation Altitude for 0 Degree Latitude Target, with the Constellation Inclination in Color. This plot shows how constellation inclination influences the objective space, with arrows indicating the optimization direction. 


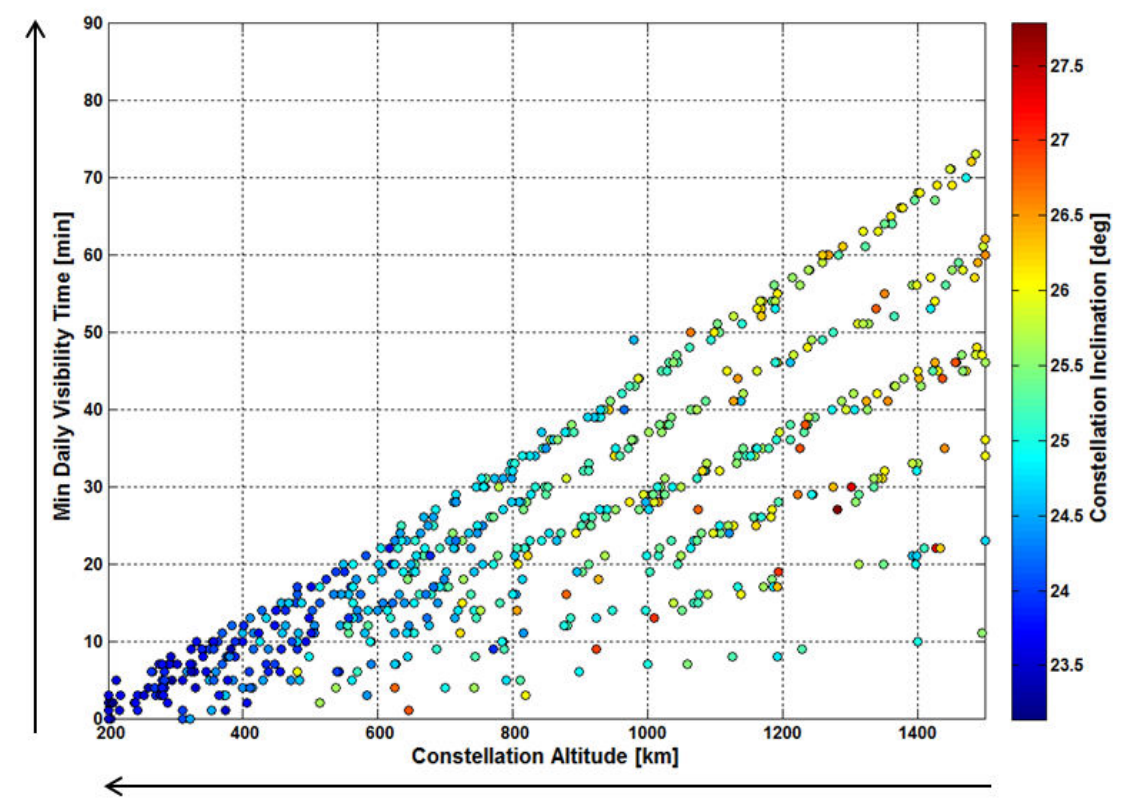

Figure 4.14. Number of Spacecraft vs. MDVT vs. Constellation Altitude for 22.5 Degree Latitude Target, with the Constellation Inclination in Color. This plot shows how constellation inclination influences the objective space, with arrows indicating the optimization direction.

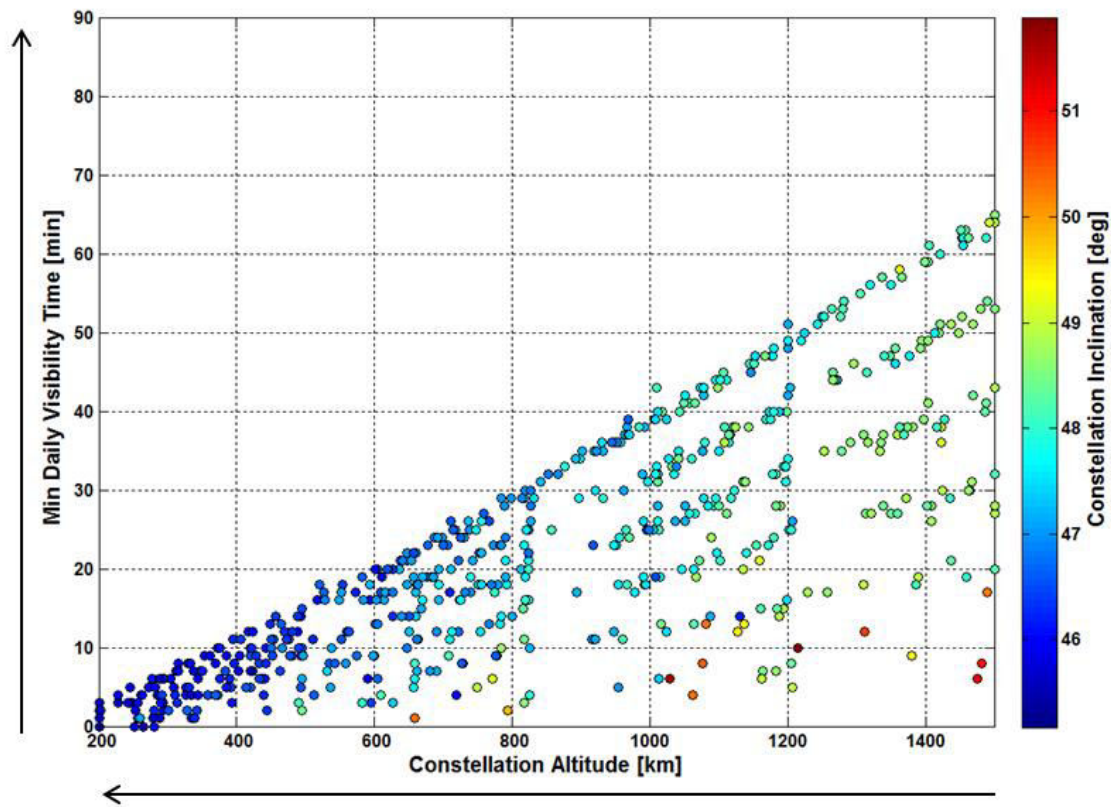

Figure 4.15. Number of Spacecraft vs. MDVT vs. Constellation Altitude for 45 Degree Latitude Target, with the Constellation Inclination in Color. This plot shows how constellation inclination influences the objective space, with arrows indicating the optimization direction. 


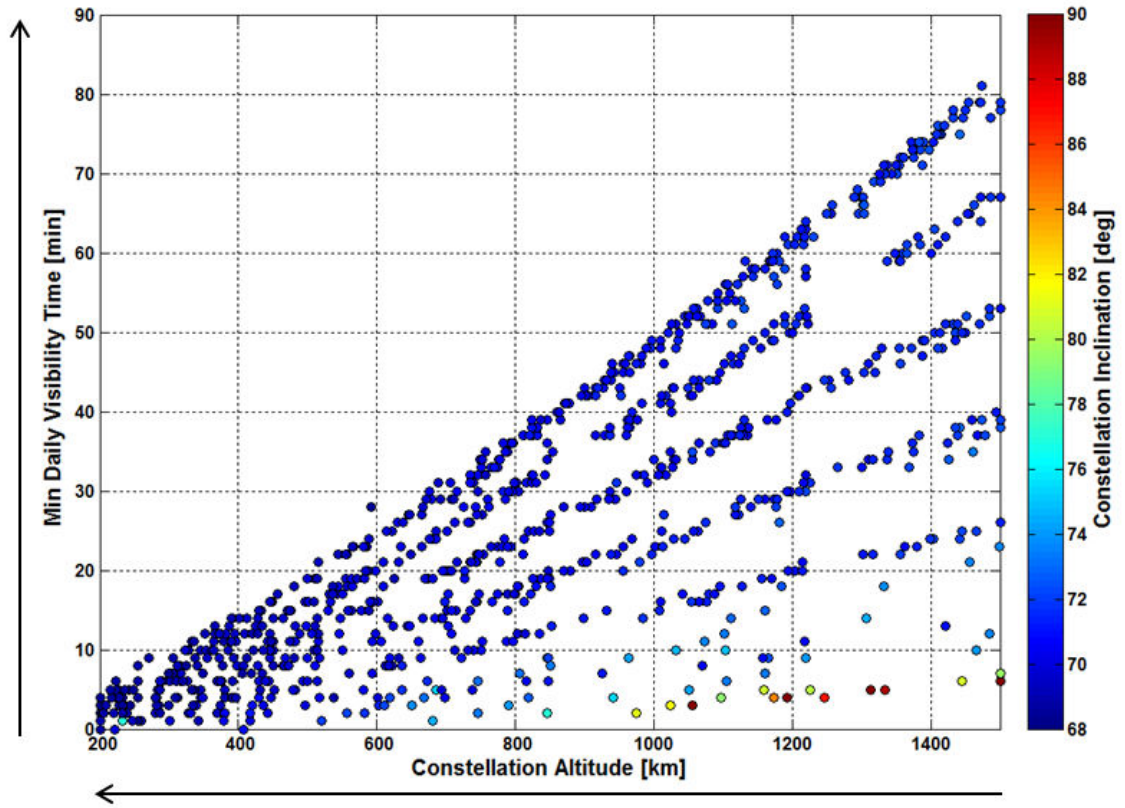

Figure 4.16. Number of Spacecraft vs. MDVT vs. Constellation Altitude for 67.5 Degree Latitude Target, with the Constellation Inclination in Color. This plot shows how constellation inclination influences the objective space, with arrows indicating the optimization direction.

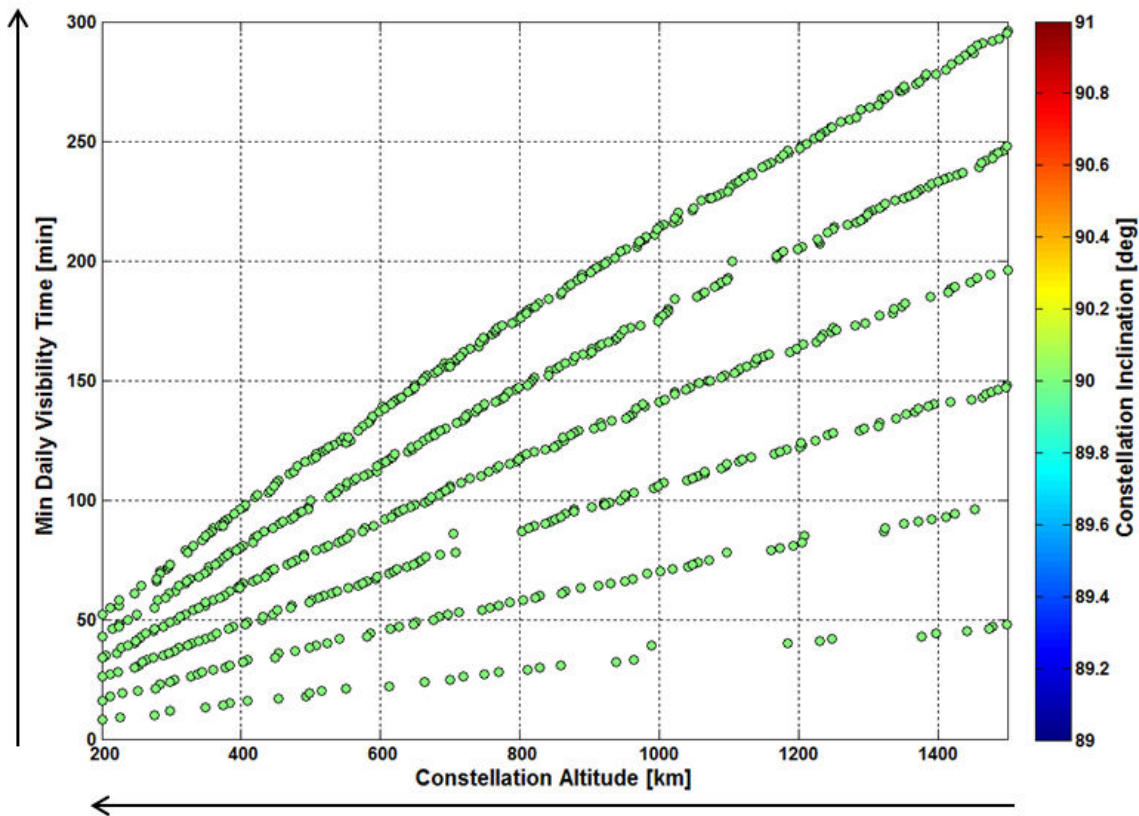

Figure 4.17. Number of Spacecraft vs. MDVT vs. Constellation Altitude for 90 Degree Latitude Target, with the Constellation Inclination in Color. This plot shows how constellation inclination influences the objective space, with arrows indicating the optimization direction. 
Several very interesting trends emerge from Fig. 4.13-4.17 by observing how the inclination of the constellation affects the objective space. These trends can be characterized as follows:

- For the target regions at 0 and 90 degrees latitude, the optimal constellation inclination is 0 and 90 degrees respectively.

- For the target regions at 22.5, 45, and 67.5 degrees latitude, the optimal constellation inclination is slightly higher than the corresponding target latitude. As the altitude of the constellation increases, the optimal inclination also increases.

- For the target region at 22.5 degrees latitude, the optimal inclination ranges from roughly 23 degrees to 27 degrees between an altitude of 200 and $1500 \mathrm{~km}$ respectively.

- For the target region at 45 degrees latitude, the optimal inclination ranges from roughly 45 to 49 degrees between an altitude of 200 and $1500 \mathrm{~km}$ respectively.

- For the target region at 67.5 degrees latitude, the optimal inclination ranges from roughly 68 to 72 degrees between an altitude of 200 and $1500 \mathrm{~km}$ respectively.

- For the target regions at 45 and 67.5 degrees latitude, there are several solutions with slightly larger inclinations for the single satellite case at higher altitudes.

Next, the objective space surrounding the MRT will be characterized with similar threedimensional plots. The following five plots, Fig. 4.18-4.22, are similar to Fig. 4.8-4.12 except they replace MDVT with MRT. The purpose of these plots is to show how the number of satellites in the constellation, the constellation altitude, and the MRT all interact in the objective space. As before, the following five plots are shown in order of increasing target region altitude. 


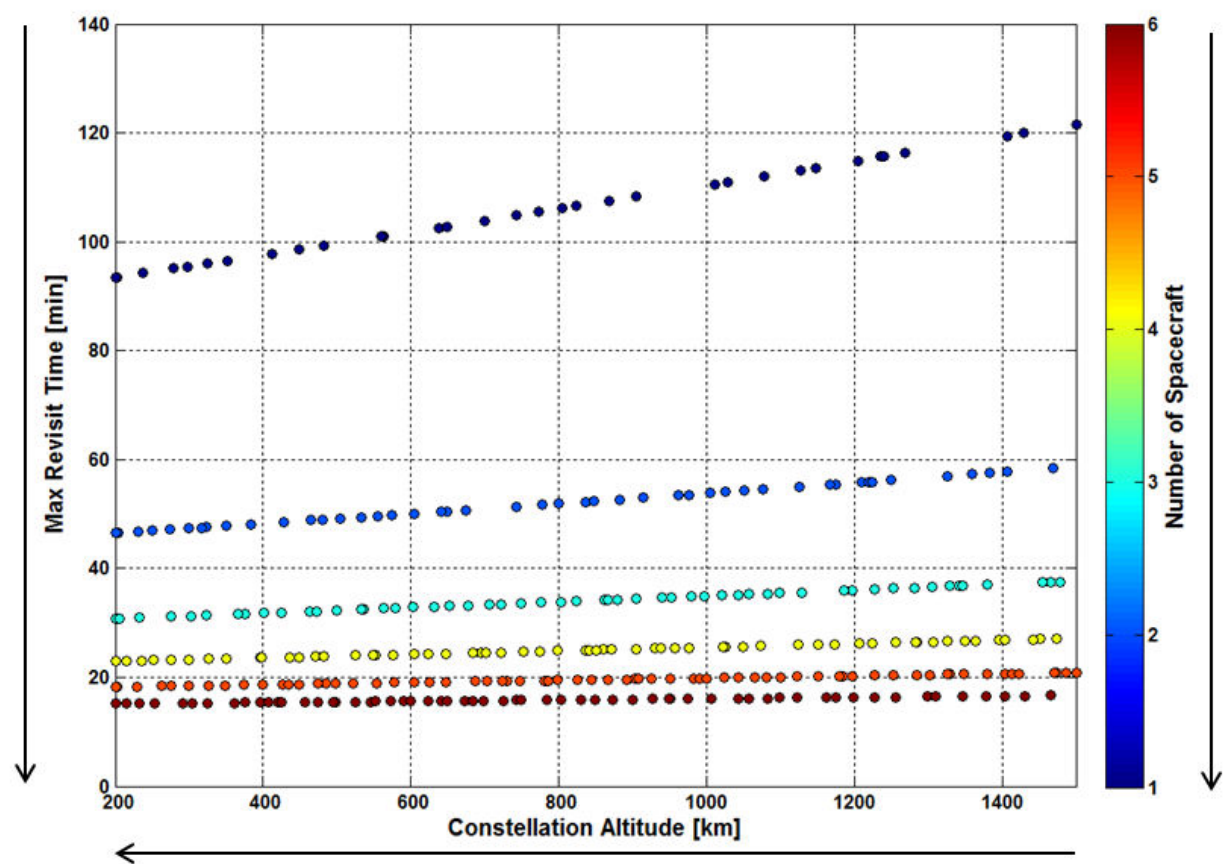

Figure 4.18. Number of Spacecraft vs. Constellation Altitude vs. MRT for 0 Degree Latitude Target. This plot shows the trends between these three objectives with the MDVT omitted. The arrows indicate the optimization direction.

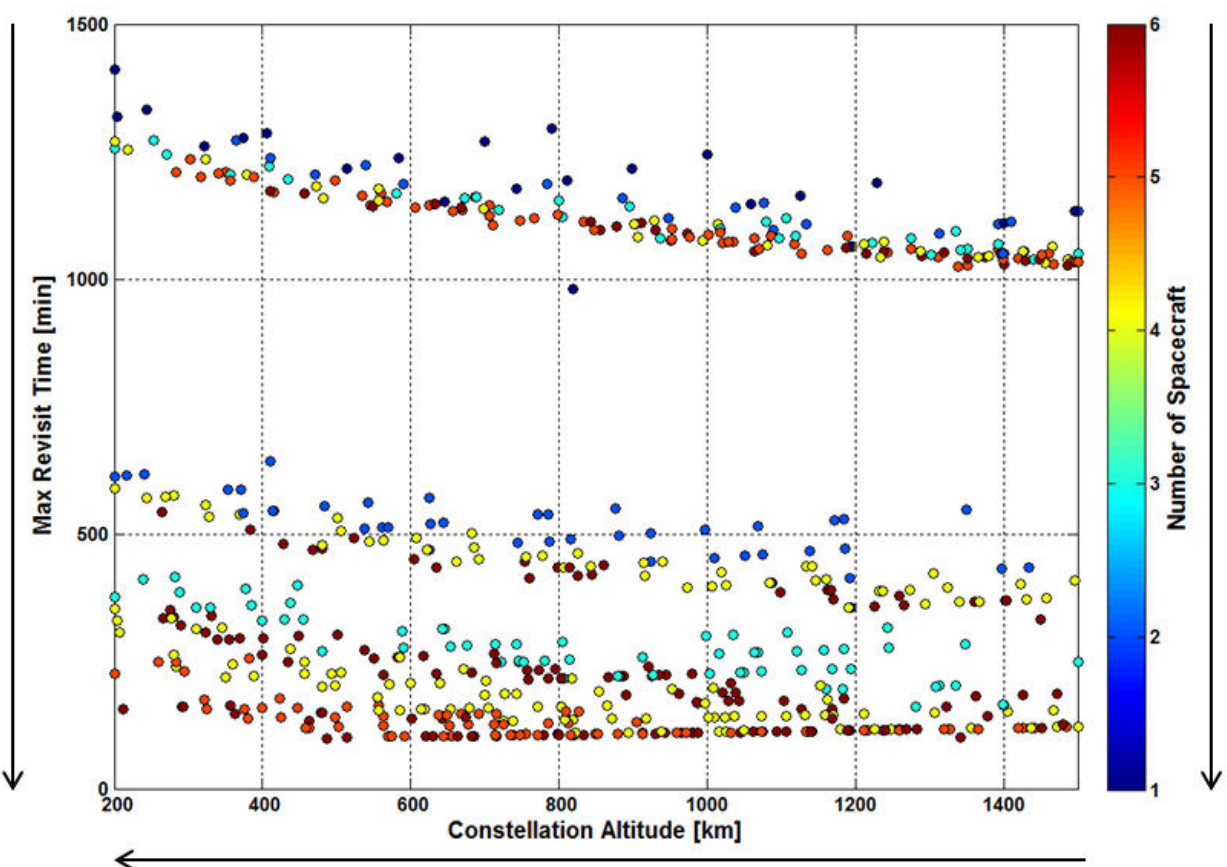

Figure 4.19. Number of Spacecraft vs. Constellation Altitude vs. MRT for 22.5 Degree Latitude Target. This plot shows the trends between these three objectives with the MDVT omitted. The arrows indicate the optimization direction. 


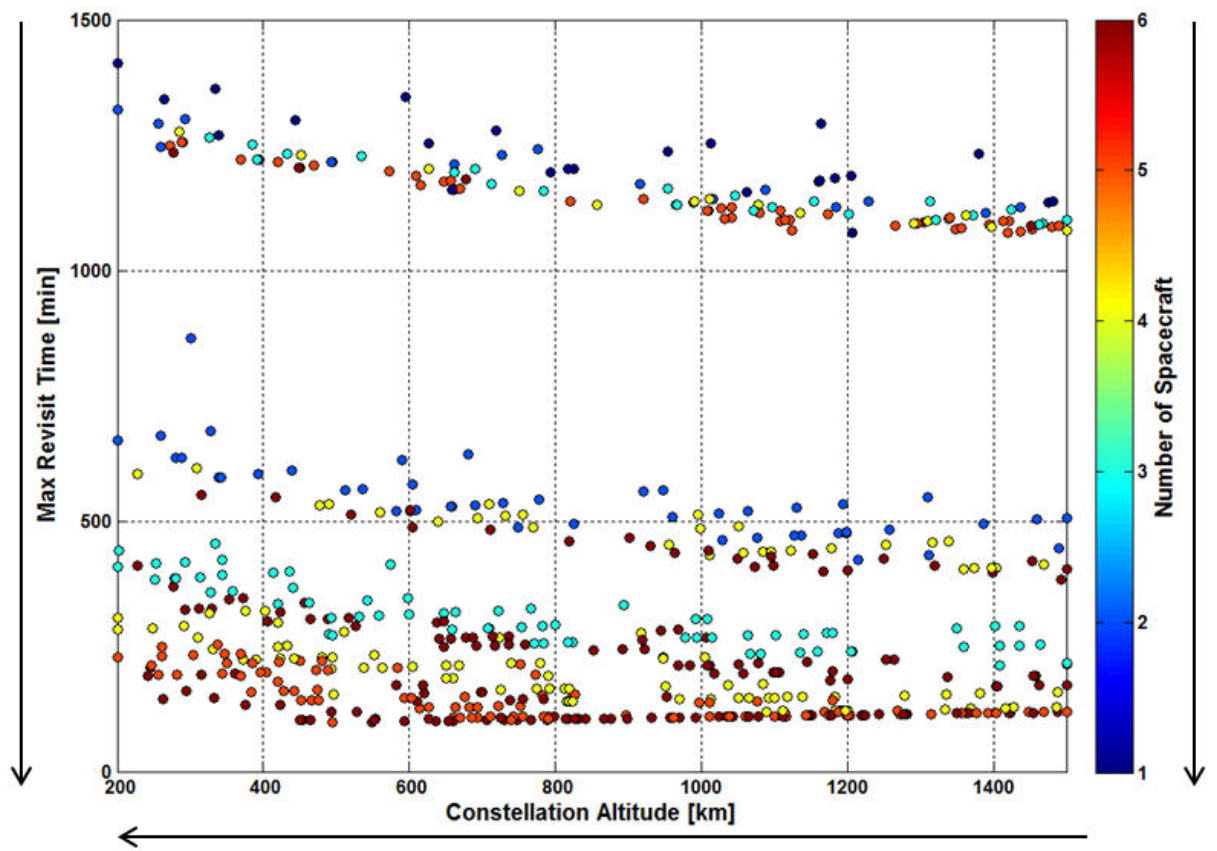

Figure 4.20. Number of Spacecraft vs. Constellation Altitude vs. MRT for 45 Degree Latitude Target. This plot shows the trends between these three objectives with the MDVT omitted. The arrows indicate the optimization direction.

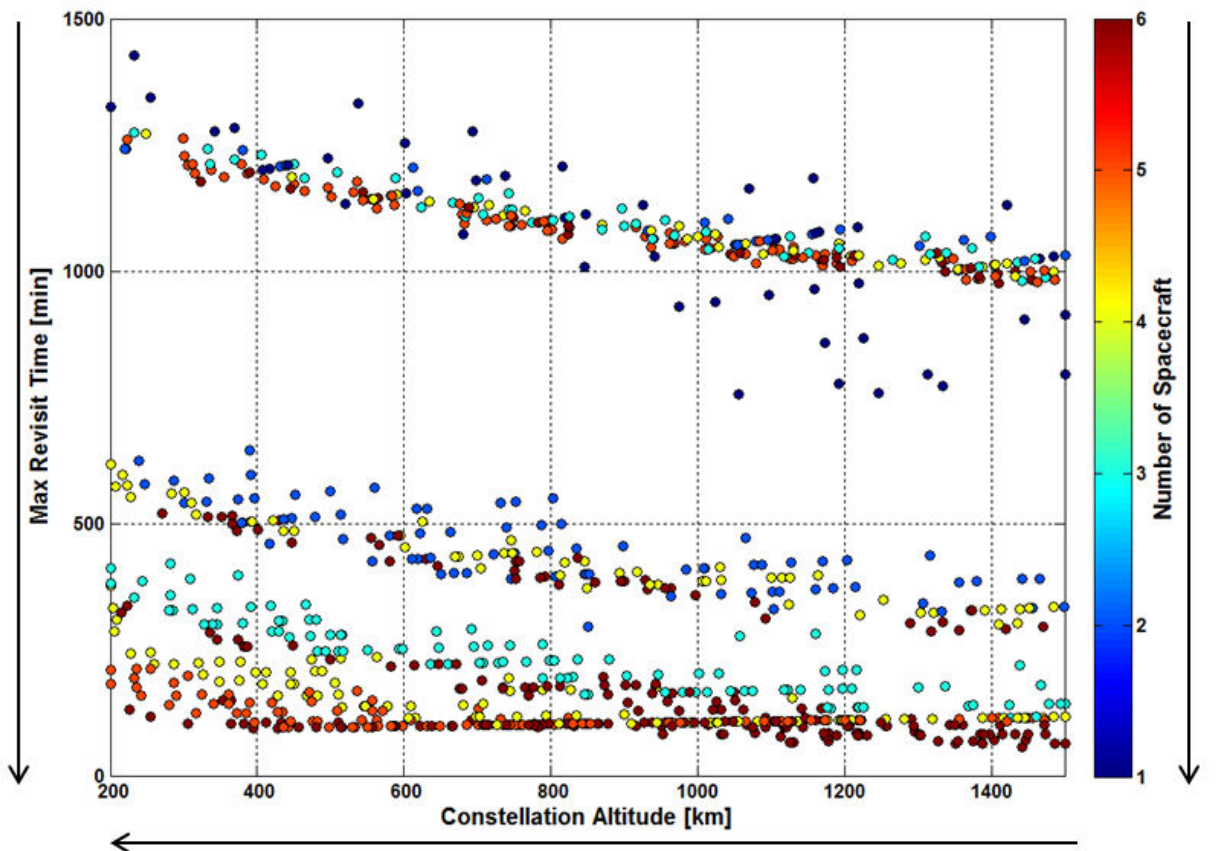

Figure 4.21. Number of Spacecraft vs. Constellation Altitude vs. MRT for 67.5 Degree Latitude Target. This plot shows the trends between these three objectives with the MDVT omitted. The arrows indicate the optimization direction. 


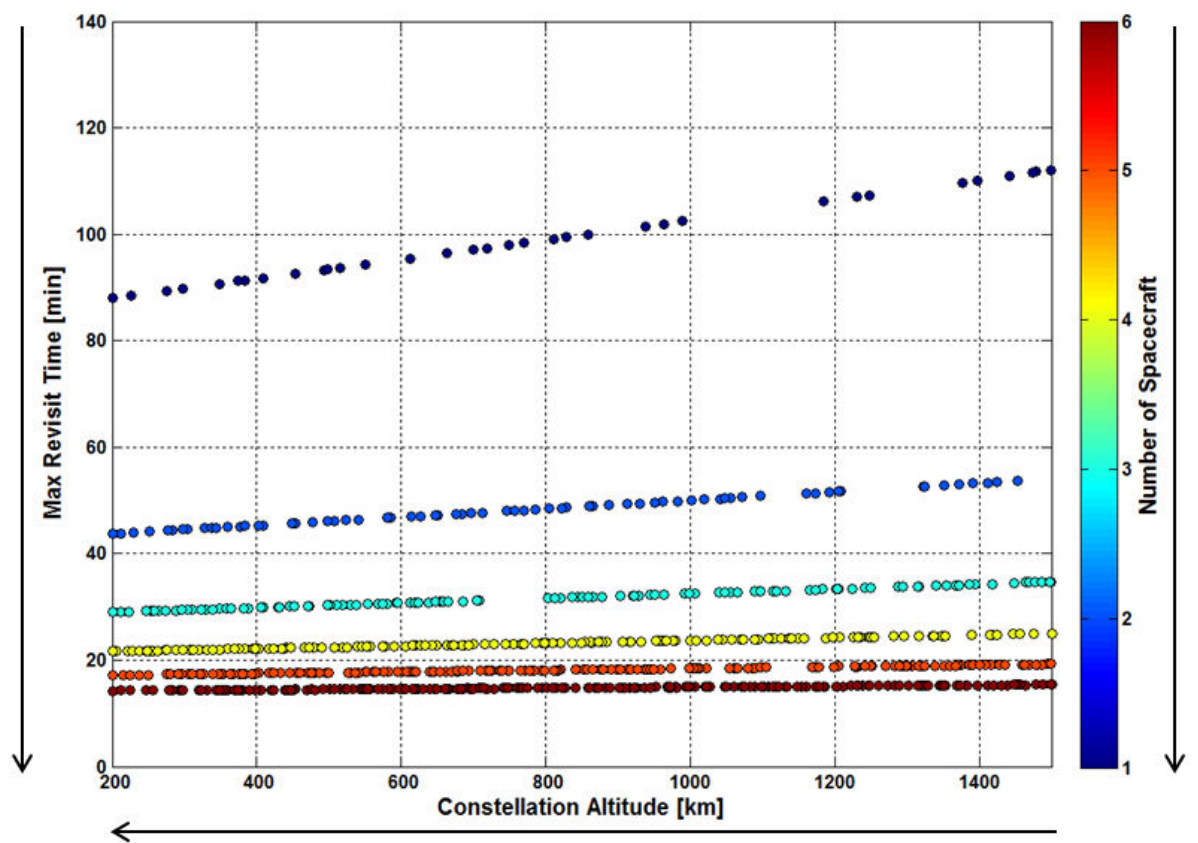

Figure 4.22. Number of Spacecraft vs. Constellation Altitude vs. MRT for 90 Degree Latitude Target. This plot shows the trends between these three objectives with the MDVT omitted. The arrows indicate the optimization direction.

Figures 4.18-4.22 show the relationships in the three dimensional objective space between the MRT, constellation altitude, and number of spacecraft. For target regions at latitudes of 0 and 90 degrees, the objective space is very clean. Here, increasing the altitude of the constellation actually decreases the maximum revisit time and degrades performance. As seen for target regions at latitudes other than 0 and 90 degrees, the objective space is extremely cluttered. It can be seen that increasing the constellation altitude in some cases results in reduced revisit times, while in other cases will result in increased revisit times. The complex objective space in these plots can be shown to be a result of the number of orbital planes. It has been discussed previously that these discontinuous groupings of designs are due to the symmetrical designs of Walker-delta patterns, given by the number of orbital planes. The following five plots, Fig. 4.23-4.27, show these trends in MRT from a different perspective, including the 
number of orbital planes. Here, the MRT is shown on the $\mathrm{x}$-axis, the number of spacecraft is shown on the y-axis, and the number of orbital planes is shown in color. Again, the following five plots show the differences in the latitude of the target region in order of increasing latitude.

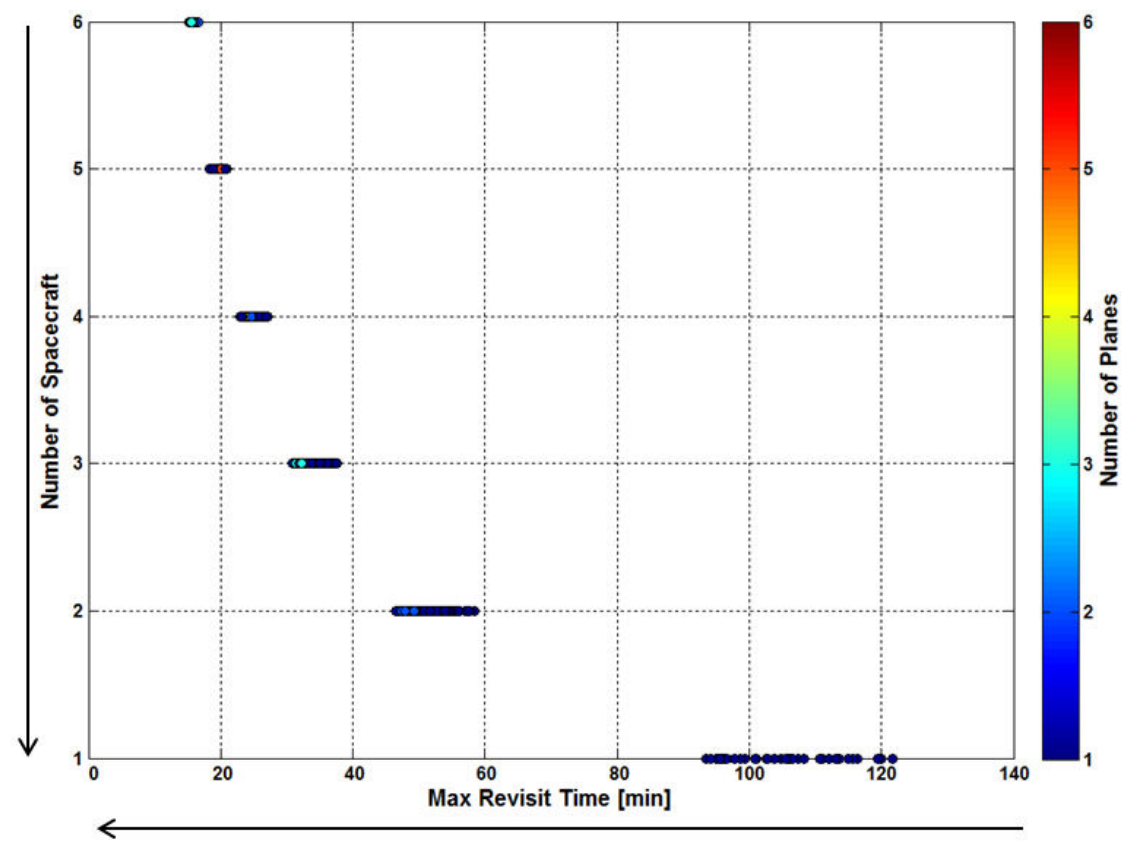

Figure 4.23. Number of Spacecraft vs. MRT for 0 Degree Latitude Target, with the Number of Planes Shown in Color. This plot shows how the number of orbital planes influences the objective space, with arrows indicating the optimization direction. 


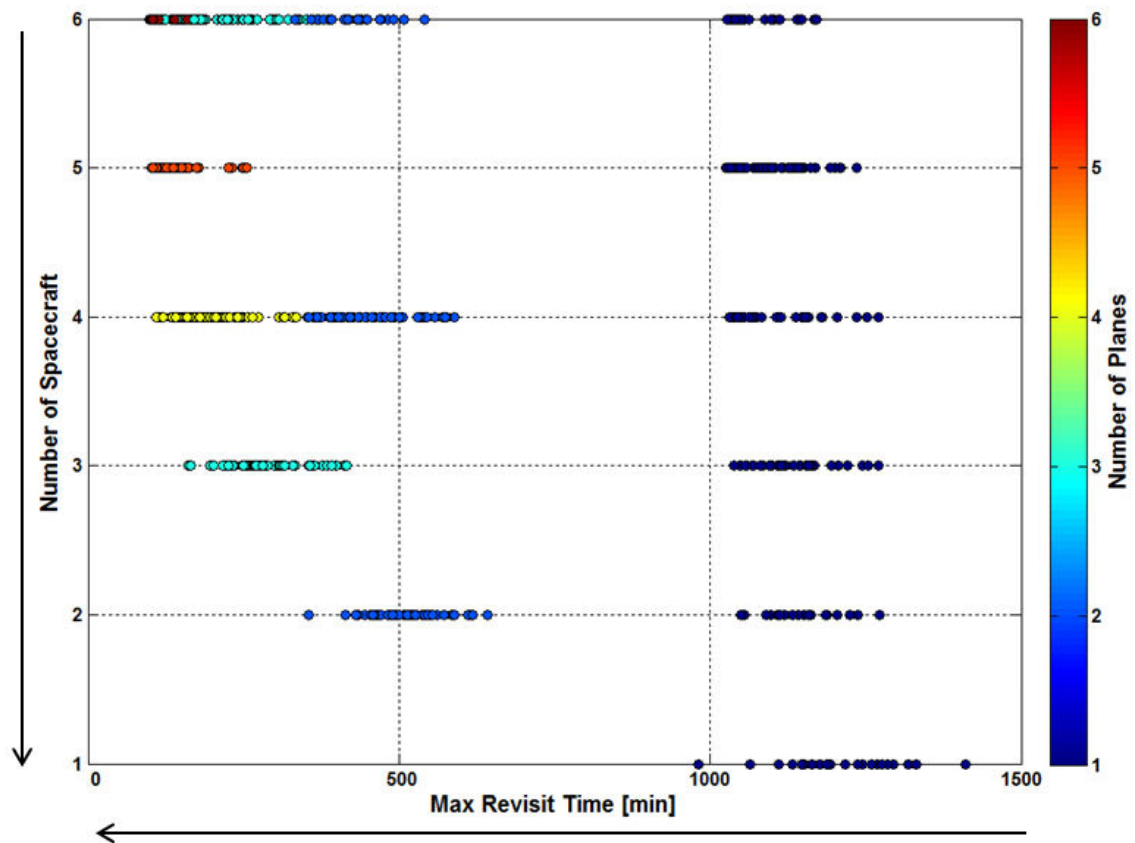

Figure 4.24. Number of Spacecraft vs. MRT for 22.5 Degree Latitude Target, with the Number of Planes Shown in Color. This plot shows how the number of orbital planes influences the objective space, with arrows indicating the optimization direction.

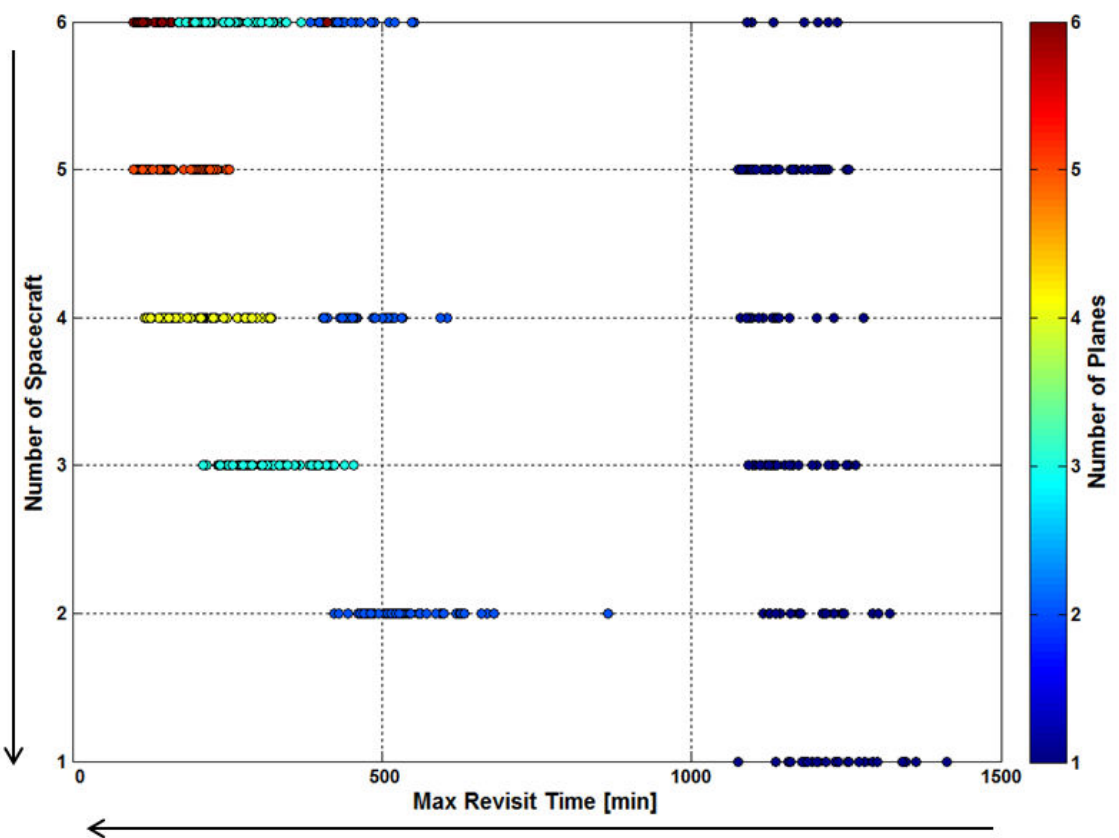

Figure 4.25. Number of Spacecraft vs. MRT for 45 Degree Latitude Target, with the Number of Planes Shown in Color. This plot shows how the number of orbital planes influences the objective space, with arrows indicating the optimization direction. 


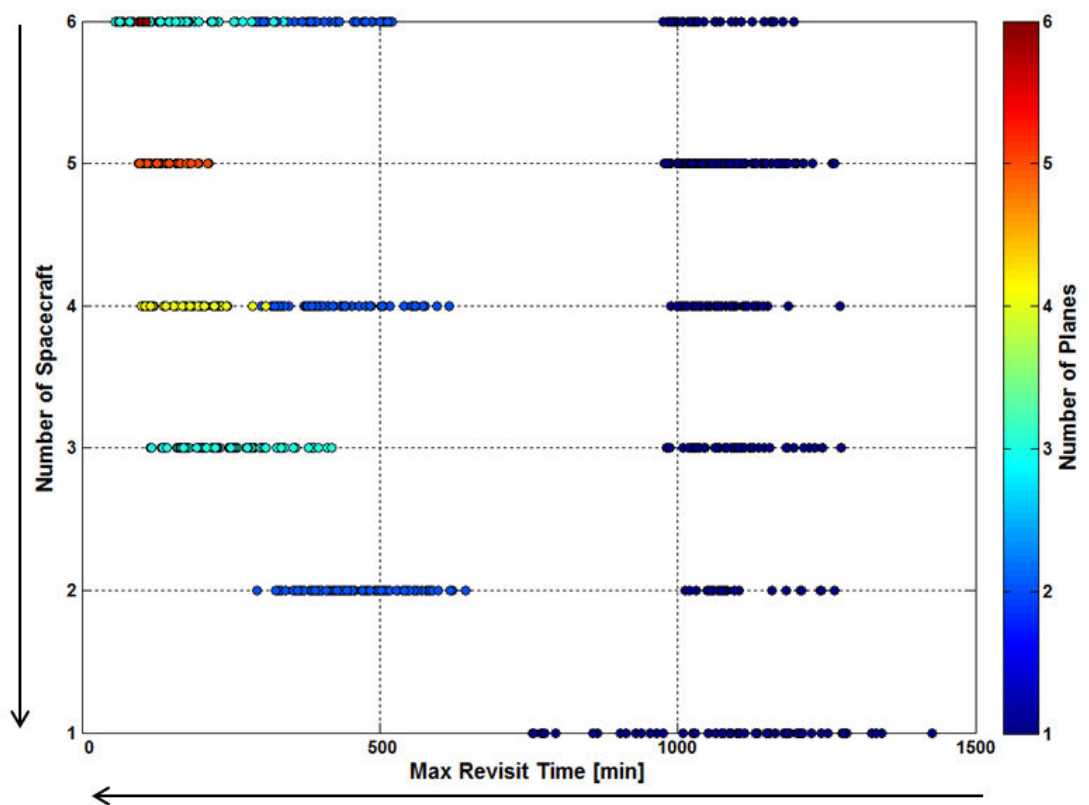

Figure 4.26. Number of Spacecraft vs. MRT for 67.5 Degree Latitude Target, with the Number of Planes Shown in Color. This plot shows how the number of orbital planes influences the objective space, with arrows indicating the optimization direction.

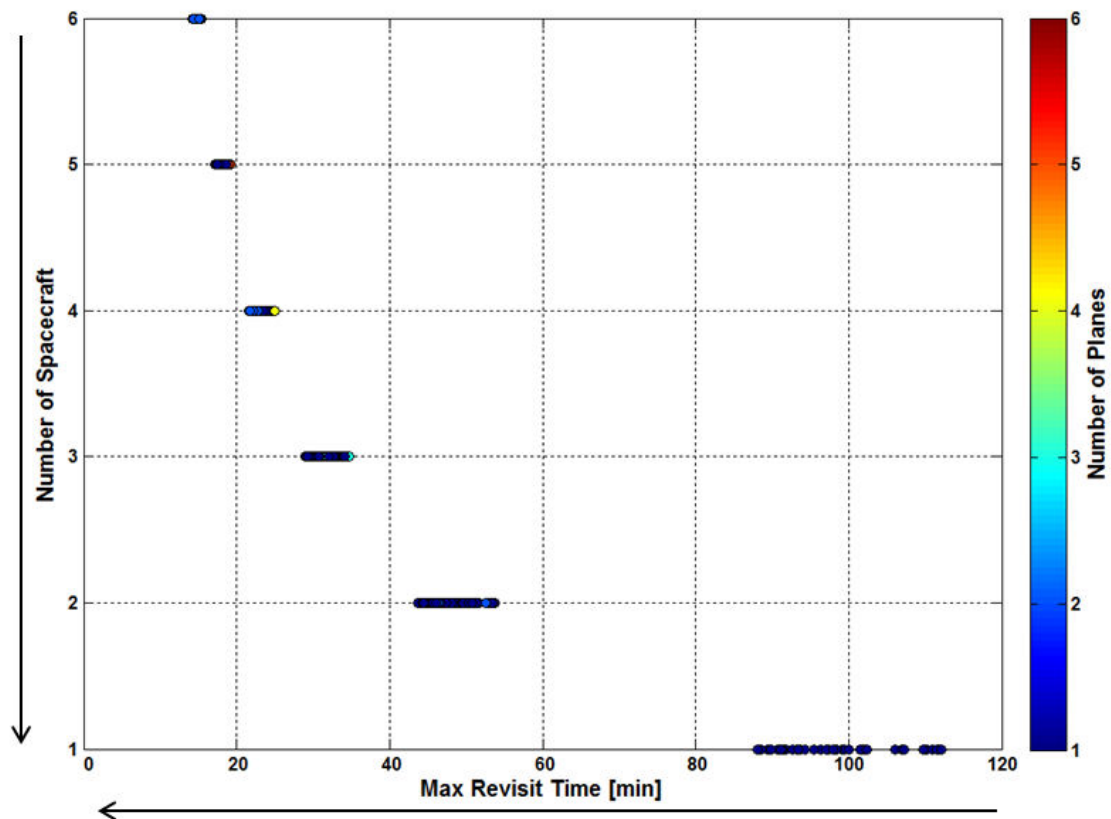

Figure 4.27. Number of Spacecraft vs. MRT for 90 Degree Latitude Target, with the Number of Planes Shown in Color. This plot shows how the number of orbital planes influences the objective space, with arrows indicating the optimization direction. 
Fig. 4.23-4.27 show how the number of orbital planes influences the objective space surrounding the MRT and number of spacecraft in the constellation. It is evident that Walkerdelta patterns produce a discontinuous objective space in the MRT due to the number of orbital planes being symmetrical and factors of the total number of spacecraft in the constellation. Several interesting trends emerge here from Fig. 4.23-4.27 which are characterized as follows:

- For target regions at latitudes near the equator or poles, the optimal number of orbital planes is one in order to reduce the MRT. This makes sense because if one orbital plane is used, the satellites will be evenly distributed in this plane and every gap in coverage will be constant. If multiple planes were used here and the satellites were asymmetrically distributed, there would be several small gaps with one large gap, and therefore the MRT would be greater than for a single plane.

- For target regions at latitudes of 22.5, 45, and 67.5 degrees latitude, increasing the number of orbital planes always reduces the MRT, resulting in increased performance.

- As the number of satellites in the constellation increases, the best achievable MRT independent of altitude, increases while increasing at a lower rate for more satellites. This indicates that it will take many more satellites than six to achieve continuous coverage in LEO for a single target point at latitudes that are somewhat far from the equator or poles.

- Similar to the MDVT performance metric, the worst achievable performance for the MRT is at a latitude of 45 degrees, followed by 22.5 degrees, and then 67.5 degrees; these three targets however are still very similar in terms of performance. 
The goals in performing this case study were to characterize the design and objective space surrounding small regional LEO remote sensing Walker constellation systems. In addition to characterizing the design and objective space, another goal was to see how the latitude of the region of interest affects the achievable performance. Both of these goals were met by providing numerous Pareto-frontiers that depicted the various trends and tradeoffs in performance for these conflicting objectives. In addition, these trends were observed for target regions at latitudes of 0 , $22.5,45,67.5$ and 90 degrees. It was shown that target regions at 0 and 90 degrees provide far increased performance over the other three mid latitudes. The 90 degree target region provided slightly improved performance over the 0 degree target region. This is most likely due to the fact that the target region on the equator is moving in a prograde motion as the Earth rotates, whereas the target region on the pole remains relatively fixed. Slightly increased coverage can therefore be maintained for target regions near the poles as opposed to target regions near the equator. Additionally, the worst achievable coverage is seen for a target region at a latitude directly in between the equator and the poles at a latitude of 45 degrees. The results of this case study confirm the original hypotheses made in addition to quantifying the achievable performance metrics associated with this type of problem. In addition, the solution spaced proved to be not smooth and very discrete in nature. This could mean that analytical techniques would have been very difficult in solving for the solution space for this type of problem. The use of numerical methods here proved to be very useful and were considered successful.

\subsection{Case 2}

The goal in performing this case study is to observe the effects of minimum ground elevation angle on both daily visibility time and revisit time, for a small LEO regional Walker 
constellation system. The previous study utilized five different target points at varying latitudes to represent small regions and show the effects of varying latitude on the performance of a constellation. This study will use a single target point at 45 degrees latitude, which was shown to have the worst achievable performance of the five target latitude points. Additionally, this study will only show the effects of minimum ground elevation angle on daily visibility time and revisit time for a constellation of 6 satellites. Two separate studies will be performed, Case $2 \mathrm{a}$ and 2 b. The objective functions in Case $2 \mathrm{a}$ are mean daily visibility time, constellation altitude, and minimum ground elevation angle. The objective functions in Case $2 \mathrm{~b}$ are mean revisit time, constellation altitude, and minimum ground elevation angle.

\subsubsection{Case 2a Problem Formulation}

The problem formulation for this case is very similar to the formulation provided for Case 1. The STK simulation parameters and the $\varepsilon$ NSGA-II tuning parameters are kept the same. The structure of the chromosome is kept the same except an $8^{\text {th }}$ gene is added at the end for the minimum ground elevation angle, $\varepsilon$, and is shown as below in Fig. 4.28. The design search space, shown in Table 4.5 is also similar to Case 1 except the minimum ground elevation angle is added and is bounded between 0 and 90 degrees. This range for the minimum ground elevation angle represents all possible angles when access between the satellite and the ground point is possible.

$$
\text { Chromosome Structure }=\left[\begin{array}{llllllll}
a & \text { inc } & \Omega_{\text {seed }} & \theta_{\text {seed }} & T & P & F & \varepsilon
\end{array}\right]
$$

Figure 4.28. Case 2 Chromosome Structure. This vector represents the 8 genes that make up the chromosome for a single individual. These are the variable parameters that are to be optimized in the ENSGA-II algorithm. 
Table 4.5. Case 2 Design Search Space.

\begin{tabular}{lccc}
\hline \hline Variable & Search Space & Value & Units \\
\hline \hline a & Bounded & $6578-7878$ & $\mathrm{~km}$ \\
ecc & Constant & 0 & - \\
inc & Bounded & $0-90$ & $\mathrm{deg}$ \\
$\mathrm{W}$ & Constant & 0 & $\mathrm{deg}$ \\
$\Omega_{\text {seed }}$ & Bounded & $0-360$ & $\mathrm{deg}$ \\
$\theta_{\text {seed }}$ & Bounded & $0-360$ & $\mathrm{deg}$ \\
$\mathrm{T}$ & Constant & 6 & - \\
$\mathrm{P}$ & Bounded & Factors of T (1-6) & - \\
$\mathrm{F}$ & Bounded & $0-(\mathrm{P}-1)$ & - \\
$\varepsilon$ & Bounded & $0-90$ & $\mathrm{deg}$
\end{tabular}

The objective functions and corresponding epsilon grid values are shown in Table 4.6. In Case 2a, the objective functions are minimum ground elevation angle, constellation altitude, and mean daily visibility time. Case 2 differs from Case 1 in that the mean performance metrics are used as opposed to the max and min performance metrics. This is done in order to get a better idea of the average effects the minimum ground elevation angle has on constellation performance.

The mean DVT is found in a similar way as the min DVT in Case 1. Instead of the lowest DVT across the entire simulation run being used, the daily visibility times for each day over the entire simulation run are averaged to obtain the mean DVT.

Table 4.6. Case 2a Design Objectives and Corresponding Epsilon Grid Values.

\begin{tabular}{ccccc}
\hline \hline Design Objective & $\begin{array}{c}\text { Optimization } \\
\text { Direction }\end{array}$ & Range & Epsilon Value & Units \\
\hline \hline Minimum Ground Elevation Angle & Maximize & $0-90$ & 2 & $\operatorname{deg}$
\end{tabular}


Constellation Altitude

Mean Daily Visibility Time

$$
\begin{array}{lc}
\text { Minimize } & 200-1500 \\
\text { Maximize } & 0-1440
\end{array}
$$$$
25
$$

$\mathrm{km}$

$\min$

\subsubsection{Case 2a Results}

First several hypotheses will be made followed by the results of this case study. It is obvious that increasing the minimum ground elevation angle will result in lower daily visibility times, although the nature of the relationship between these two is unknown. This is due to the fact that with higher minimum ground elevation angles, the chance of viewing the target region is simply reduced.

Although Case 2a represents a single simulation run, the resulting Pareto-frontier will be shown from several different angles and perspectives in order to better visualize the resulting trends. Note that each point in the plot represents an entire constellation design that is characterized by a given number of satellites, each with its own unique orbital elements.

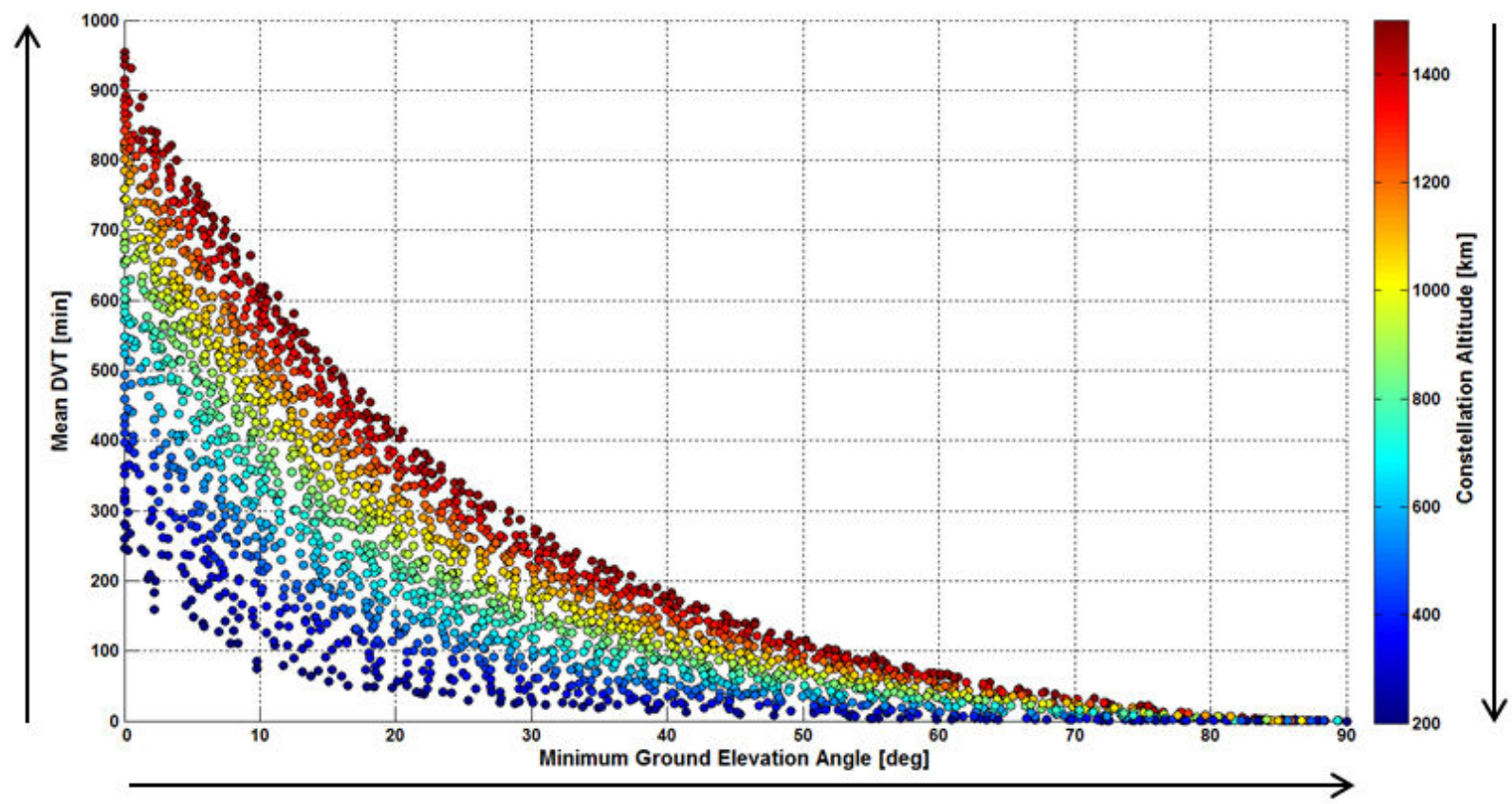

Figure 4.29. Minimum Ground Elevation Angle vs. Constellation Altitude vs. Mean DVT for Target Region at 45 Degrees Latitude. Note that the arrows represent the optimization direction. 


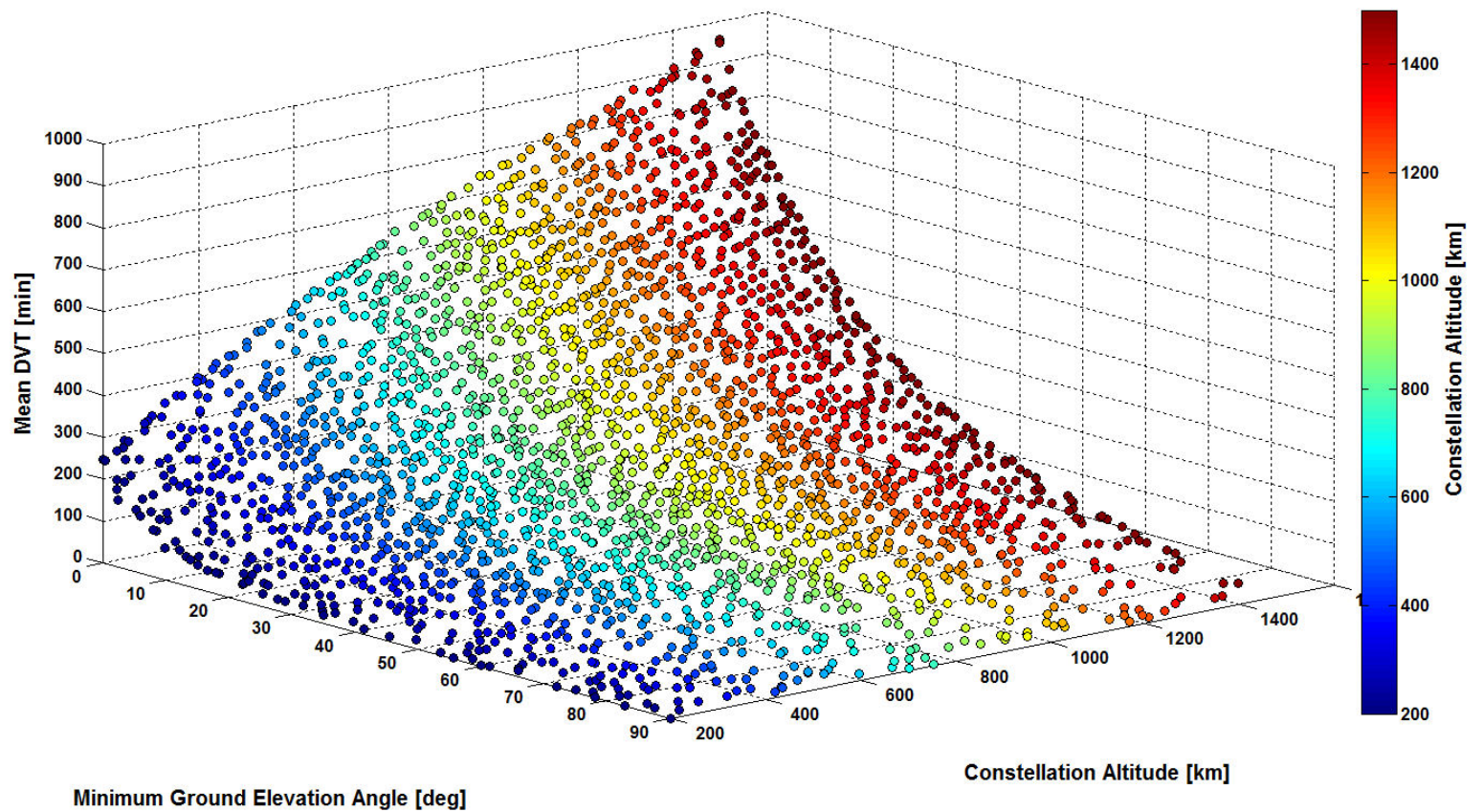

Figure 4.30. Minimum Ground Elevation Angle vs. Constellation Altitude vs. Mean DVT for Target Region at 45 Degrees Latitude. Note that the Constellation Altitude is represented on both an axis and in color.

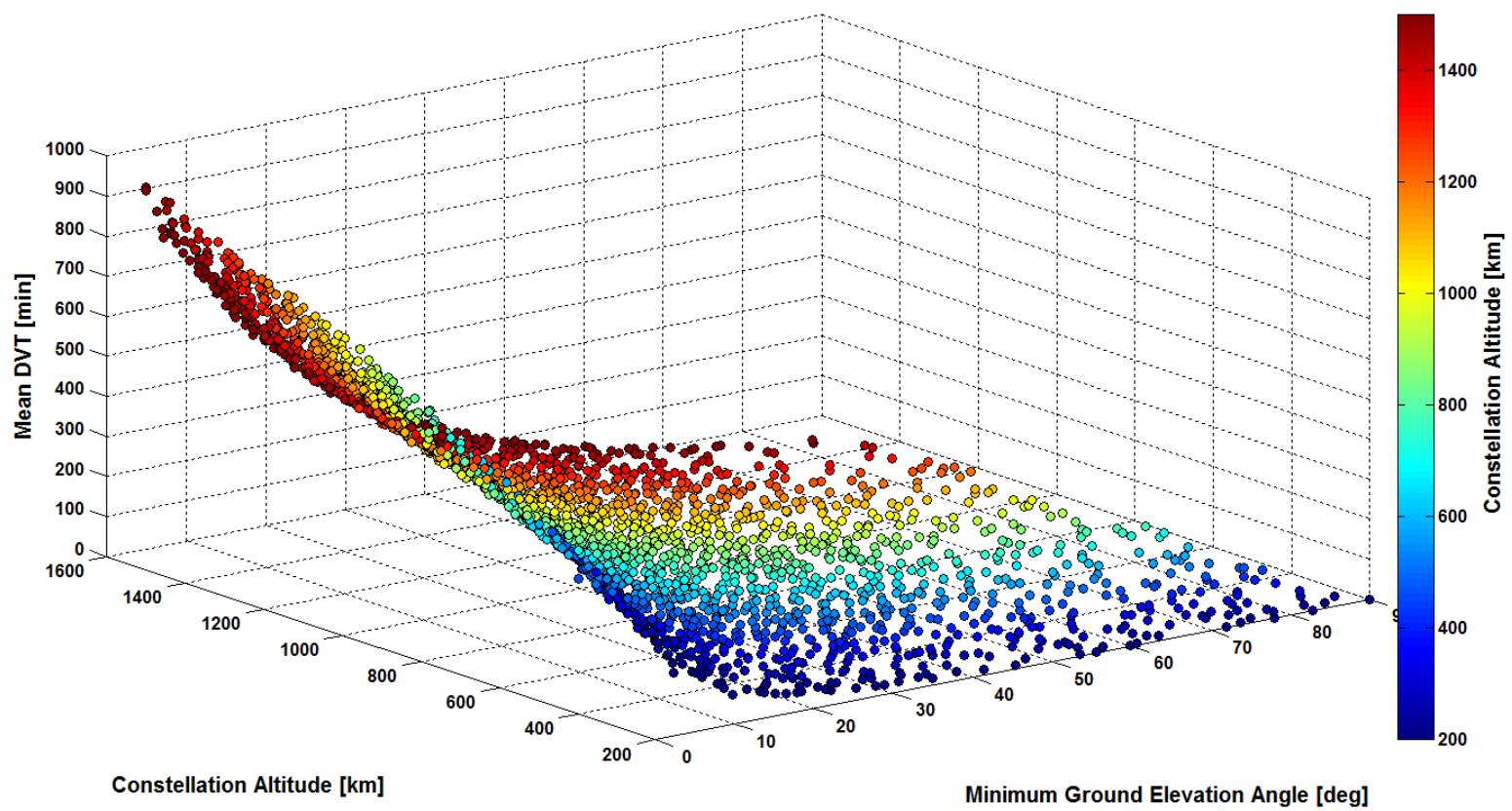

Figure 4.31. Minimum Ground Elevation Angle vs. Constellation Altitude vs. Mean DVT for Target Region at 45 Degrees Latitude. Note that the Constellation Altitude is represented on both an axis and in color. 
Figures 4.29-4.31 show the effect of minimum ground elevation angle and constellation altitude on the mean DVT. Several basic trends emerge which are characterized as follows:

- As the altitude of the constellation increases, the mean DVT also increases in a near linear nature for a given minimum ground elevation angle.

- The best achievable mean DVT is given by high constellation altitudes at low ground elevation angles.

- The Pareto-frontier for this six-satellite constellation in this objective space appears to be continuous in nature, with the frontier being a smooth thin surface.

- As the minimum ground elevation angle increases, the mean DVT decreases for a given altitude. This relationship is non-linear in nature; for low minimum ground elevation angles the slope is steep and for high minimum ground elevation angles the slope is nearly flat. This means there is a large difference between constellation performance between 0 and 10 degree minimum ground elevation angles, and a small difference between constellation performance between 80 and 90 degree minimum ground elevation angles.

- The spread in mean DVT between 200 and $1500 \mathrm{~km}$ is roughly 700 minutes for a minimum ground elevation angle of 0 degrees. The spread in mean DVT between 200 and $1500 \mathrm{~km}$ is roughly 150 minutes for a minimum ground elevation angle of 45 degrees. The results of this case study confirm the original hypotheses in addition to providing an insight into the nature of the relationship between these three performance metrics. It should be noted however, that the smoothness of the solution space indicates that it may have been possible to obtain these results through analytical methods as opposed to the numerical techniques used. 
Analytical methods could possibly be quicker at obtaining solutions to this problem, as well as similar problems, in addition to providing exact solutions.

\subsubsection{Case 2b Problem Formulation}

The problem formulation for Case $2 \mathrm{~b}$ is identical to Case $2 \mathrm{a}$ with one difference. The mean DVT objective function is simply replaced with the mean revisit time. Again, the mean revisit time is found in a similar way as the max revisit time in Case 1. Instead of the largest gap being found, all of the gaps in the simulation run are averaged together to obtain the mean revisit time. The chromosome structure, optimization variables, and design search space are identical to those in Case 2a. The design objectives and epsilon grid values are shown in Table 4.7. In this case, the mean revisit time is actually constrained to a maximum value of 1440 minutes, or a single day. At very high elevation angles, the mean revisit time will be several days to weeks, and these designs are thus omitted.

Table 4.7. Case 2b Design Objectives and Corresponding Epsilon Grid Values.

\begin{tabular}{lcccc}
\hline \hline \multicolumn{1}{c}{ Design Objective } & $\begin{array}{c}\text { Optimization } \\
\text { Direction }\end{array}$ & Range & Epsilon Value & Units \\
\hline \hline Minimum Ground Elevation Angle & Maximize & $0-90$ & 2 & $\mathrm{deg}$ \\
Constellation Altitude & Minimize & $200-1500$ & 25 & $\mathrm{~km}$ \\
Mean Revisit Time & Minimize & $0-1440$ & 10 & $\mathrm{~min}$
\end{tabular}

\subsubsection{Case 2b Results}

It is hypothesized that increasing the minimum ground elevation angle will reduce the average revisit time. The nature of the relationship between the minimum ground elevation angle and the mean revisit time for a given altitude is however unknown. It is expected that as 
the minimum ground elevation angle increases, the average revisit time will increase exponentially in nature.

The following Pareto-frontier shown in Fig. 4.32 depicts how the minimum ground elevation angle and constellation altitude impact the mean revisit time for a constellation of 6satellites and a target region at a latitude of 45 degrees. Figure 4.33 shows a small section of the entire Pareto-frontier shown in Fig. 4.32; the minimum ground elevation angle is zoomed in to show the region between 0 and 45 degrees. Note that each point in the plot represents an entire constellation design that is characterized by a given number of satellites, each with its own unique orbital elements.

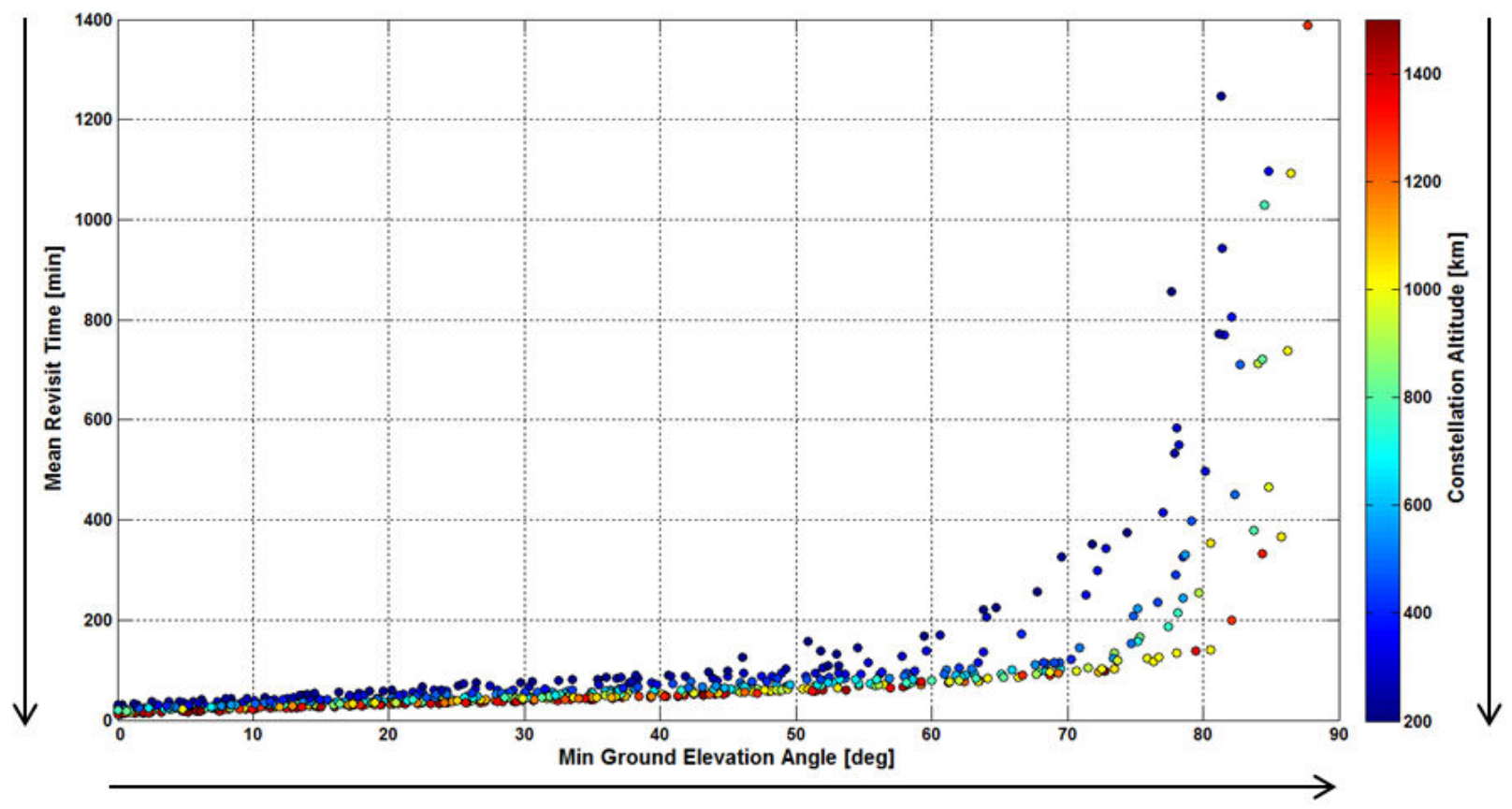

Figure 4.32. Minimum Ground Elevation Angle vs. Constellation Altitude vs. Mean Revisit Time for Target Region at 45 Degrees Latitude. Note that the arrows represent the optimization direction. 


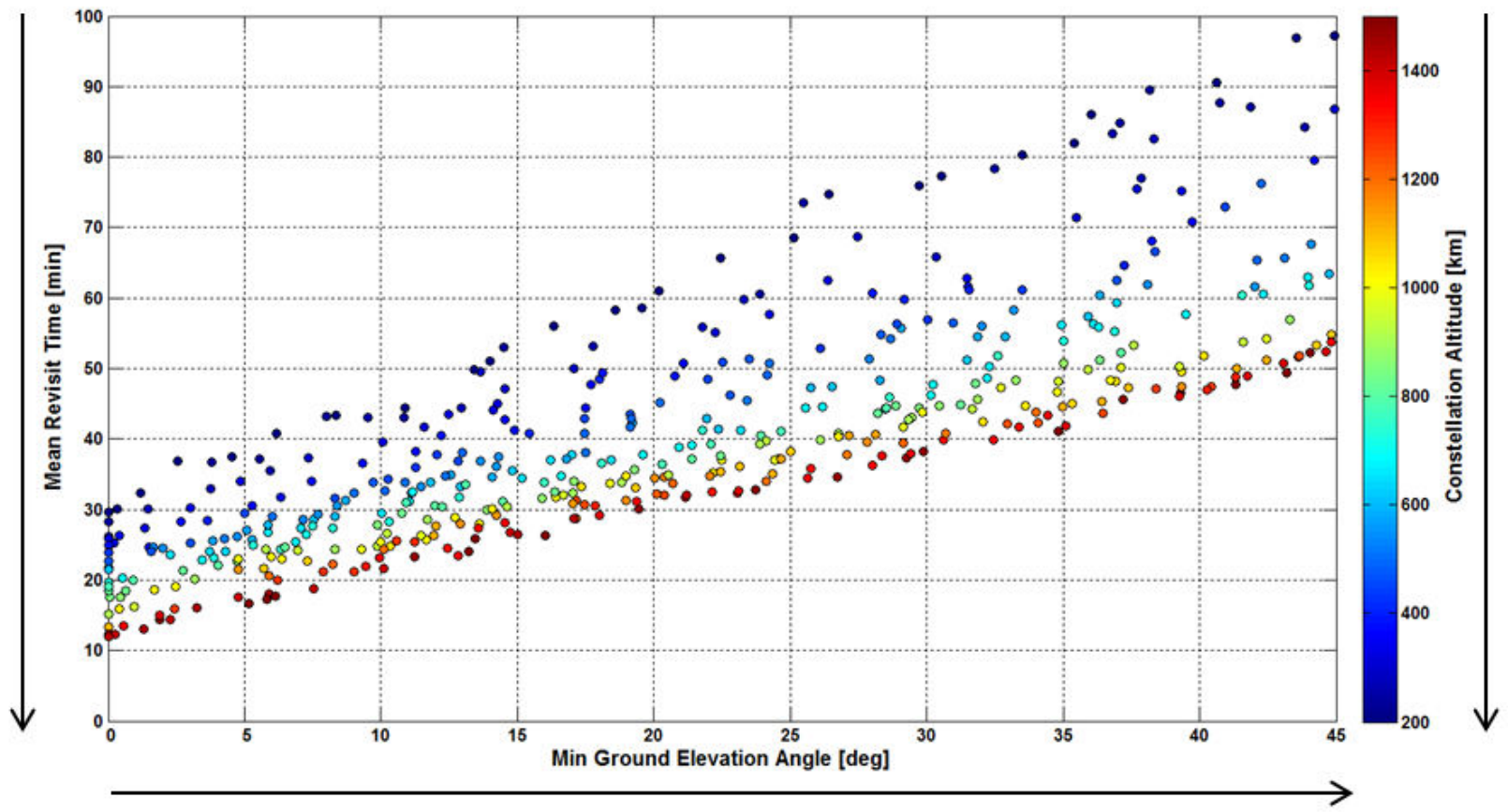

Figure 4.33. Minimum Ground Elevation Angle vs. Constellation Altitude vs. Mean Revisit Time for Target Region at 45 Degrees Latitude. This plot shows a small section of the entire Pareto-frontier, where the minimum ground elevation angle is shown between 0 and 45 degrees. Note that the arrows represent the optimization direction.

The Pareto-frontier shown in this case exhibits several interesting trends which are characterized as follows:

- As the minimum ground elevation angle increases, the mean revisit time also increases. The relationship between minimum ground elevation angle and mean revisit time is linear in nature for minimum ground elevation angles between 0 and roughly 45 degrees. Above a 45 degree minimum ground elevation angle, the mean revisit time increases more rapidly as the minimum ground elevation angle increases, and is seemingly exponential in nature.

- The spread in mean revisit time between 200 and $1500 \mathrm{~km}$ altitude is roughly 20 minutes at a 0 degree minimum ground elevation angle and is roughly 50 minutes at a 45 degree minimum ground elevation angle. 
- The best achievable mean revisit time for a six-satellite Walker constellation and a target region at 45 degrees latitude is at an altitude of $1500 \mathrm{~km}$ and a minimum ground elevation angle of 0 degrees.

The results of this case study confirm the hypothesis made, in addition to providing quantifiable insight into the relationship between these metrics. As seen in Case 2a, it may have been possible to use analytical methods in finding the solution space for Case $2 \mathrm{~b}$.

\subsection{Case 3}

The goal of this case study is to see how relaxing several of the constraints on the traditional symmetric Walker-delta pattern will affect the achievable performance of the constellation for a similar setup as in Case 1; a small regional LEO remote sensing constellation system. In this case study, the symmetric Walker-delta pattern will be applied alongside a more relaxed constellation pattern in order to characterize the differences in the traditional design and a more unconstrained design. A single target region at 45 degrees latitude will be used in this study, the number of satellites in the constellation will be fixed at 6 , and the minimum ground elevation angle is fixed at 60 degrees. The design objectives are max revisit time (MRT), min daily visibility time (MDVT), and constellation altitude.

\subsubsection{Case 3 Problem Formulation}

The problem formulation for this case is very similar to the formulation provided for Case 1. The STK simulation parameters and the ENSGA-II tuning parameters are kept the same. Two separate runs are performed in this case with very different chromosome structures and 
genes which will be discussed shortly. The design objectives are the same for both simulation runs, and are given in Table 4.8.

Table 4.8. Case 3 Design Objectives and Corresponding Epsilon Grid Values.

\begin{tabular}{lcccc}
\hline \hline \multicolumn{1}{c}{ Design Objective } & $\begin{array}{c}\text { Optimization } \\
\text { Direction }\end{array}$ & Range & Epsilon Value & Units \\
\hline \hline Constellation Altitude & Minimize & $200-1500$ & 25 & $\mathrm{~km}$ \\
Max Revisit Time & Minimize & - & 1 & $\mathrm{~min}$ \\
Min Daily Visibility Time & Maximize & $0-1440$ & 1 & $\mathrm{~min}$
\end{tabular}

For the Walker-delta pattern design space, the chromosome structure is identical to that in Case 1. For reference the chromosome structure and mapping will be presented here in Fig. 4.34. Additionally, Table 4.9 shows the design search space for the Walker-delta Pattern.

$$
\begin{aligned}
& \text { Chromosome Structure }=\left[\begin{array}{lllllll}
a & \text { inc } & \Omega_{\text {seed }} & \theta_{\text {seed }} & T & P & F
\end{array}\right]
\end{aligned}
$$

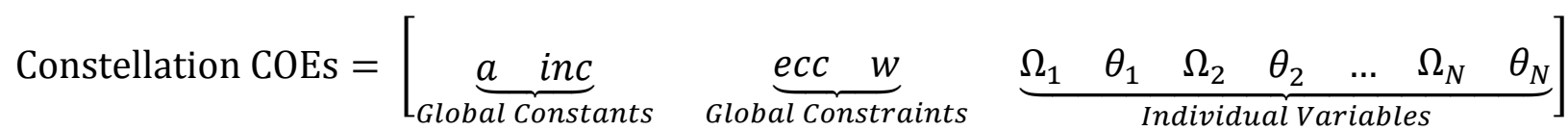

Figure 4.34. Case 3 Chromosome Structure and Resulting Makeup of Constellation COEs for the WalkerDelta Pattern. Here the variables to be optimized are represented in the chromosome structure. These variable result in corresponding orbital elements for each satellite in the constellation, which is shown as the constellation COEs.

Table 4.9. Case 3 Design Search Space for the Walker-Delta Pattern.

\begin{tabular}{lccc}
\hline \hline Variable & Search Space & Value & Units \\
\hline \hline a & Bounded & $6578-7878$ & $\mathrm{~km}$ \\
ecc & Constant & 0 & - \\
inc & Bounded & $0-90$ & $\mathrm{deg}$ \\
$\mathrm{W}$ & Constant & 0 & $\mathrm{deg}$ \\
$\Omega_{\text {seed }}$ & Bounded & $0-360$ & $\mathrm{deg}$ \\
$\theta_{\text {seed }}$ & Bounded & $0-360$ & $\mathrm{deg}$ \\
$\mathrm{T}$ & Constant & 6 & - \\
$\mathrm{P}$ & Bounded & Factors of T (1-6) & - \\
$\mathrm{F}$ & Bounded & $0-(\mathrm{P}-1)$ & -
\end{tabular}


For the relaxed constellation design space the altitude, inclination, eccentricity, and argument of perigee will be the same for all satellites in the constellation. The right ascension of the ascending node and the mean anomaly for each satellite will be allowed to vary in any manner. This is different from the Walker-delta pattern where the right ascension of the ascending node and the mean anomaly are fixed to symmetric patterns that are evenly spaced over 360 degrees as defined by the Walker-delta pattern. For the relaxed constellation design space, the altitude and inclination make up the first two genes of the chromosome, which is the same for the Walker-delta pattern. The eccentricity and argument of perigee will both be fixed at 0 , which is also the same for the Walker-delta pattern. Since the number of satellites in the constellation is fixed at 6 , there will be 1 gene for the right ascension of the ascending node and 1 gene for the mean anomaly for each of the six satellites. There will therefore be $2+2 * 6$, or 14 genes, that compose the chromosome for the relaxed constellation design space, as shown in Fig. 4.35. Additionally, Table 4.10 shows the design search space for the relaxed constellation design space.

$$
\begin{aligned}
& \text { Chromosome Structure }=\left[\begin{array}{lllllllll}
a & \text { inc } & \Omega_{1} & \theta_{1} & \Omega_{2} & \theta_{2} & \ldots & \Omega_{N} & \theta_{N}
\end{array}\right], \quad \text { where } N=6
\end{aligned}
$$

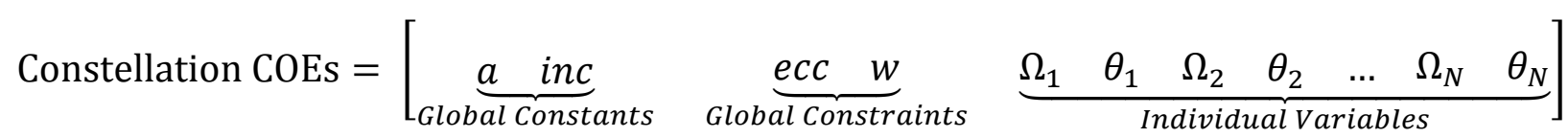

Figure 4.35. Case 3 Chromosome Structure and Resulting Makeup of Constellation COEs for the Relaxed Constellation Design. Here the variables to be optimized are represented in the chromosome structure. These variable result in corresponding orbital elements for each satellite in the constellation, which is shown as the constellation COEs. 
Table 4.10. Case 3 Design Search Space for the Relaxed Constellation Design.

\begin{tabular}{lccc}
\hline \hline Variable & Search Space & Value & Units \\
\hline \hline a & Bounded & $6578-7878$ & $\mathrm{~km}$ \\
ecc & Constant & 0 & - \\
inc & Bounded & $0-90$ & deg \\
w & Constant & 0 & deg \\
$\Omega_{\mathrm{i}}$ & Bounded & $0-360$ & $\mathrm{deg}$ \\
$\theta_{\mathrm{i}}$ & Bounded & $0-360$ & $\mathrm{deg}$
\end{tabular}

A single target point at 45 degrees latitude will be used in this study, the number of satellites in the constellation will be fixed at 6 , and the minimum ground elevation angle is fixed at 60 degrees. These two simulation runs will be run independently, and the resulting Paretofrontiers will be compared in order to distinguish differences in performance between the traditional symmetric Walker-delta pattern and the relaxed constellation pattern. Due to the nature of the problem formulation, the relaxed constellation design actually contains the set of all potential solutions in the design and objective space for the Walker-delta design, in addition to solutions that are not permissible with the Walker-delta design.

\subsubsection{Case 3 Results}

It is expected that the relaxed Walker-delta pattern will perform very similarly to the Walker-delta pattern, without the discontinuous breaks due to the symmetric nature of the Walker-delta pattern. The relaxed Walker-delta pattern may exhibit slightly improved performance over the symmetrical pattern, but the two should be very similar in nature.

The following two Pareto-frontiers show how the Walker-delta constellation pattern and the relaxed design pattern perform for providing coverage to a single target point at 45 degrees 
latitude, with a minimum ground elevation angle of 60 degrees, and for 6 satellites in the constellation. Figure 4.36 shows the tradeoff in altitude, min daily visibility time, and max revisit time for the symmetric Walker-delta pattern. Figure 4.37 shows the tradeoff in altitude, min daily visibility time, and max revisit time for the relaxed design pattern. Note that each point in the plot represents an entire constellation design that is characterized by a given number of satellites, each with its own unique orbital elements.

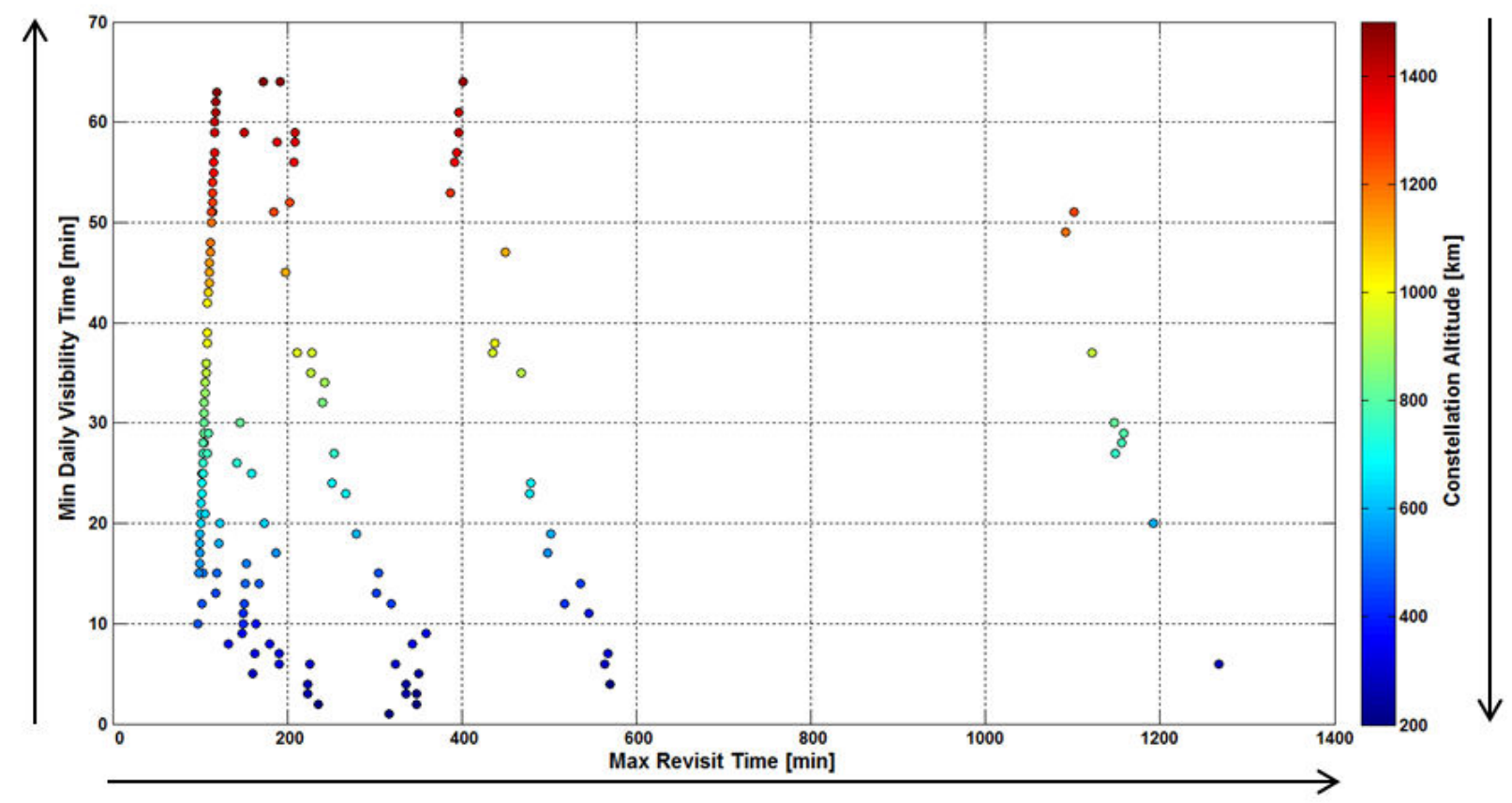

Figure 4.36. Constellation Altitude vs. MRT vs. MDVT for the Walker-Delta Pattern. This three-dimensional Pareto-frontier shows the achievable performance of a Walker-delta constellation design for a target point at 45 degrees latitude and a minimum ground elevation angle of 60 degrees. 


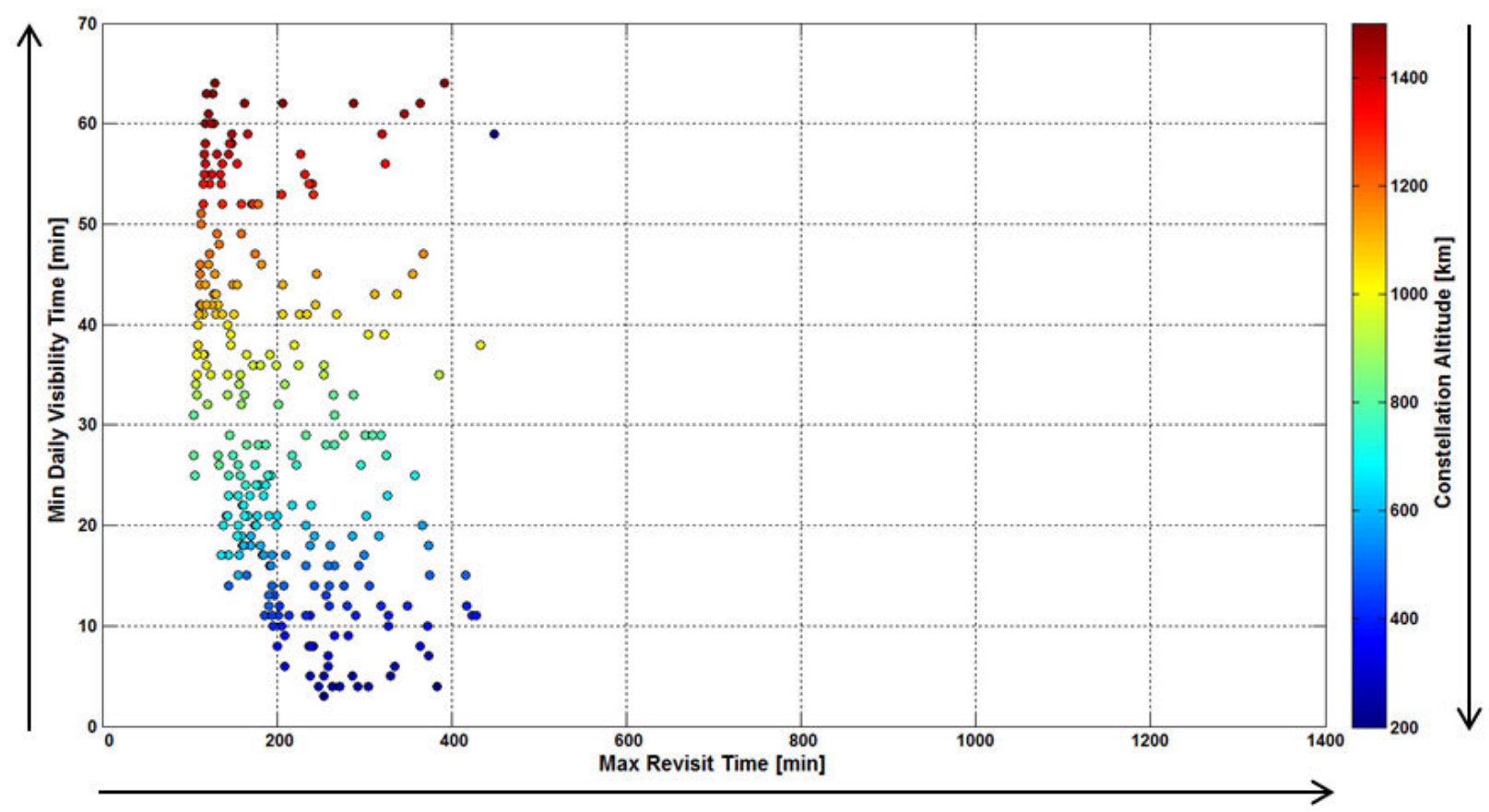

Figure 4.37. Constellation Altitude vs. MRT vs. MDVT for the Relaxed Constellation Design. This threedimensional Pareto-frontier shows the achievable performance of a relaxed constellation design for a target point at 45 degrees latitude and a minimum ground elevation angle of 60 degrees. Note that the scale of the axes is kept identical to that in Fig. 4.36.

The Pareto-frontiers presented above for both cases show several interesting results. The basic trends and tradeoffs have been discussed previously for the Walker-delta pattern; most notably you can see discrete groups based on 1,2,3, and 6 symmetrical orbital planes. Similar trends are seen for the relaxed constellation design, without these discrete groupings. The Pareto-frontier for the relaxed constellation design space is more continuous than the discontinuous Walker-delta design space. If asymmetric designs are permitted, as in the relaxed constellation design space, a more continuous Pareto-frontier will be obtained. It is also important to note that roughly the same performance is achieved between the traditional Walkerdelta pattern and the relaxed constellation design pattern. This means that Walker-delta patterns perform very well for the small regional coverage LEO constellation design problem. Note 
however, that the Walker-delta pattern performs poorly in revisit time when there is no diversity in the number of orbital planes, which is expected.

The hypotheses of this case study match the observed results. It was seen that the performance between the two designs are very similar in nature, and the relaxed Walker-delta configuration is more continuous. Although the performance is very similar, it is important to note that the optimal design space may differ which could provide significant advantages for a particular design. It is important to remember that the solution space alone cannot be used to determine whether or not the Walker-delta pattern versus the relaxed design space provided a more optimal design. Although the two solutions spaces are nearly identical, the design space between the two may vary significantly. Upon further investigation however, the optimal relaxed design variables showed striking similarities to the optimal Walker-delta design variables, thereby resulting in similar constellation geometries between the two designs. It can therefore be concluded that the Walker-design pattern provides a good initial solution to this problem, where further investigation may yield slightly improved results. 


\section{FUTURE WORK}

This thesis provided a characterization of a very small problem relating to satellite constellation design. As such, there is still a lot of work to be accomplished in this field. As this thesis studied how the latitude of the region of interest affected the performance of the constellation, similar work in this area has yet to be accomplished. It would be interesting to see how the size or even the shape of the target region would affect the coverage. It would also be interesting to study additional objective functions such as delta-v for stationkeeping. Since the only orbital perturbation used in this study was the $\mathrm{J} 2$ perturbation, it would be interesting to include additional perturbations and try to optimize the delta- $v$ with these additional perturbations in mind. As constellation systems become more achievable, small satellites or CubeSats might be used to fulfill mission goals. As such, it would be interesting to place constraints on the constellation design space to study specific designs in line with these constraints. This thesis focused heavily on remote sensing constellation systems by utilizing a minimum ground elevation angle of 60 degrees. It would be interesting to study other types of constellation systems for either regional or even zonal coverage. Finally, it would be very interesting to apply an evolutionary algorithm to more specific designs that have actually been flown to see if they could account for increased performance. While evolutionary algorithms provide a very useful tool in solving these types of problems, it would also be interesting to consider analytical methods in some instances where the solution space is smooth and continuous in nature. The constellation design problem is seemingly infinite in nature, and there are surely additional studies that need to be performed in order to better characterize this problem. As additional studies are performed, the use of evolutionary algorithms is very well placed to solve these types of problems. 


\section{LESSONS LEARNED}

In order to aid people performing similar studies in future work, several suggestions may be made related to this study or creating a thesis in general.

- When starting a thesis, understand and accept that your thesis topic and the direction of your thesis may change as you make new discoveries. These discoveries may come from researching various literature similar to your thesis or from working on your thesis and obtaining results.

- It is essential to understand every aspect of the evolutionary algorithm and know how every single parameter and function impacts the computation time and resulting solution space of the specific problem you are trying to solve. The best way to do this is to write your own algorithm from scratch and experiment with these different parameters and functions. I could not stress enough how important it is to have a full understanding of the algorithm you are using.

- When implementing an evolutionary algorithm to obtain Pareto-frontiers, try to utilize the most powerful computing hardware available to you, and utilize parallel-processing to reduce computation time if possible.

- Try to understand how the simulation time span, step size, and grid spacing affects the solution space. It is imperative to understand the affects these parameters have on the solutions space so that you do not mischaracterize the final solutions.

- Spend time thinking about whether or not the obtained solutions make sense. Often the most powerful tool available to you is your own common sense. 
- Do not place constraints on the design space unless you know for certain the effects that will have in limiting the solution space. Often times the best solutions are ones that are the opposite of what you might expect.

- Be sure to carefully select the epsilon grid sizes for the various objective functions. A grid size that is too large will result in a Pareto-frontier that does not fully characterize the objective space. Similarly, a grid size that is too small will result in long computation times that may not reach the true optimal solutions.

- Make sure to consider the optimal design space in addition to the optimal solution space for a solution to the entire problem. When comparing two different design spaces to a common solution space, the design variable must be taken into account when trying to determine the optimal solution. 


\section{CONCLUSION}

The constellation design problem for providing coverage to a small target region, with 16 satellites in a Walker-delta pattern, a minimum ground elevation angle of 60 degrees, and in LEO has been characterized in this study. This problem provides a good basis for a small remote sensing constellation system providing coverage to a specific region on the Earth. The achievable performance of this type of system has been discussed for target regions at $0,22.5$, 45, 67.5, and 90 degrees latitude. Pareto-frontiers were developed showing constellation performance including the number of satellites, orbital altitude, revisit times, and daily visibility times at each of these latitudes. In addition, the impact of varying the minimum elevation angle on both the daily visibility times and revisit times was shown for a target region at 45 degrees latitude and a constellation of 6 satellites. Several restrictions on the Walker-delta pattern were relaxed in order to see how more asymmetrical designs solve this constellation design problem. The obtained Pareto-frontiers were then compared between Walker-delta patterns and relaxed Walker-delta patterns for a target region at 45 degrees latitude, with a 60 degree minimum elevation angle, and for a constellation of 6 satellites. All of the obtained Pareto-frontiers were displayed and the trends were characterized in the results section of this thesis. The main results of this study are:

- Although several solutions may be lost by restricting the design space to Walker-delta patterns, these patterns provide very good coverage to the small regional LEO constellation design problem in terms of daily visibility time and revisit time. Most notably, significant computational savings are achieved by restricting the design to the symmetrical Walker-delta patterns because only 7 genes are needed to fully characterize

a constellation design. If anything, the Walker-delta pattern provides an extremely good 
baseline to approach the small regional LEO constellation problem. In addition, the Walker-delta pattern is very symmetric in nature which provides perhaps the best solution in terms of maintaining constellation geometry over time, and thus reducing the required fuel for stationkeeping. Walker patterns rely on common altitude, common inclination, and circular orbits among all of the satellites in the constellation. This can be extremely important to maintain a constant geometry, and thus maintain constant performance. It is also efficient to have all of the satellites in several planes at a common inclination and altitude in terms of launch costs.

- It is shown that the Walker-delta pattern produces a very discontinuous Pareto-frontier in the objective space surrounding the min daily visibility time, max revisit time, constellation altitude, and number of spacecraft. This was found to be a result of the symmetrical design of the Walker-delta pattern itself.

- Perhaps the most important trends observed were that the altitude of the constellation has a significant impact on the min daily visibility time, whereas the number of orbital planes has a significant impact on the max revisit time. By increasing the altitude of the constellation, the min daily visibility time is increased. By providing diversity in the number of orbital planes, the max revisit time may be reduced.

- Increasing the number of satellites in the constellation provided better daily visibility times as well as revisit times. Going from five to six satellites in the constellation provided a much larger improvement in daily visibility time, whereas it provided very little increase in the revisit time.

- The optimal inclination of the constellation was found to be 0 degrees if the target region is visible at a given altitude from a satellite with an orbital plane in the equatorial plane. 
Therefore for a target region at 0 degrees latitude, the optimal inclination of the constellation is 0 degrees, for both increasing the daily visibility time and reducing the revisit time. Similarly, if the target region is near the poles, the optimal inclination will be 90 degrees.

- The optimal inclination of the constellation was found to vary with altitude for target regions in between the equator and the poles. The optimal inclination is always slightly greater than the target latitude, and increases with increasing altitude.

- The minimum ground elevation angle has a significant impact on the performance of the constellation, for both daily visibility time and revisit time. Obviously, lower minimum ground elevation angles provide better daily visibility times and revisit times. It was found that the daily visibility time is greatly affected by changing the minimum ground elevation angle at low angles, whereas the revisit time was greatly affected by changing the minimum ground elevation angle at high angles.

- The symmetric nature of the Walker-delta pattern was relaxed to show how a more relaxed constellation design could improve performance. It was found that the relaxed constellation design resulted in a more continuous Pareto-frontier. The Walker-delta pattern still provided very similar performance. The optimal design variables between the two designs proved to be very similar, with similar constellation geometries produced.

- In addition to the Pareto-frontiers being characterized and different trends discussed, actual values for the maximum revisit time and minimum daily visibility time were obtained. This performance was quantified in the Pareto-frontier plots and shown in the results section. 
The Pareto-frontiers shown in this study were obtained with a variation of the $\varepsilon$ NSGA-II algorithm, which proved to be extremely effective at solving this type of constellation design problem. In addition, STK proved to be a very useful tool in assessing the access between a satellite and a target region on the ground. 


\section{REFERENCES}

1 Abdelkhalik, Osama Mohamed Omar. Orbit Design and Estimation for Surveillance Missions Using Genetic Algorithms. PhD Thesis, College Station: Texas A\&M University, 2005, 12311235.

2 Abdelkhalik, Ossama, and Ahmed Gad. "Optimization of Space Orbits Design for Earth Orbiting Missions." Acta Astronautica, 2011: 1307-1317.

3 Analytical Graphics Inc. Integrating STK and MATLAB. Flyer, Exton, PA: AGI, n.d.

4 —. "STK Programming Interface 10.1.1." AGI Online Resources. June 2014. http://www.agi.com/resources/help/online/stkDevKit/10.1.1/index.html (accessed 2014).

5 Asvial, M., R. Tafazolli, and B. G Evans. "Genetic Hybrid Satellite Constellation Design." AIAA, 2003: $1-6$.

6 Asvial, M., R. Tafazolli, and B. G. Evans. "Non-GEO Satellite Constellation Design with Satellite Diversity Using Genetic Algorithm." AIAA, 2002: 12-15.

7 Baker, R.M.L. Astrodynamics: Applications and Advanced Topics. Academic Press Inc, 1967.

8 Bandyopadhyay, Sanghamitra, and Sriparna Saha. Unsupervised Classification: Similarity Measures, Classical and Metaheuristic Approaches, and Applications. West Bengal: Springer, 2013.

9 Bate, Roger R., Donald D. Mueller, and Jerry E. White. Fundamentals of Astrodynamics. New York: Dover Publications, Inc., 1971.

10 Bruccoleri, Christian. Flower Constellation Optimization and Implementation. $\mathrm{PhD}$ Thesis, College Station: Texas A\&M University, 2007.

11 Chan, Serena, Ayanna T. Samuels, Nirav B. Shah, Jennifer E. Underwood, and Olivier L. de Weck. "Optimization of Hybrid Satellite Constellations Using Multiple Layers and Mixed Circular-Elliptical Orbits." AIAA, 2004: 9-12.

12 Coello, Carlos A., and Gary B. Lamont. Applications of Multi-objective Evolutionary Algorithms. World Scientific, 2004.

13 de Weck, Olivier L., Serena Chan, Ayanna T. Samuels, Nirav B. Shah, and Jennifer E. Underwood. "Optimization of Hybrid Satellite Constellations Using Multiple Layers and Mixed Circular-Elliptical Orbits." AIAA, 2004: 9-12.

14 de Weck, Olivier, Richard de Neufville, and Mathieu Chaize. "Staged deployment of Communications Satellite Constellations in Low Earth Orbit." Journal of Aerospace Computing, Information, and Communcation, 2004: 119-136. 
15 Deb, K., A. Pratap, S. Agarwal, and T. Meyarivan. "A Fast and Elitist Multiobjective Genetic Algorithm: NSGA-II." Journal of Evolutionary Computation, 2002: 182-197.

16 Deb, Kalyanmoy. Multi-Objective Optimization Using Evolutionary Algorithms: An Introduction. KanGAL Report, Kanpur: Indian Institute of Technology Kanpur, 2011.

17 Deb, Kalyanmoy, and N. Srinivas. "Multiobjective Optimization Using Nondominated Sorting in Genetic Algorithms." Journal of Evolutionary Computation, 1994: 221-248.

18 DeWeck, Olivier L. Multiobjective Optimization: History and Promise. Paper, Cambridge: Dept. of Aeronautics \& Astronautics, MIT, 2004.

19 Eiben, A. E., and J. E. Smith. Introduction to Evolutionary Computing. New York: Springer, 2003.

20 Ferringer, Matthew P. General Framework for the Reconfiguration of Satellite Constellations. PhD Thesis, University Park: Pennsylvania State University, 2009.

21 Ferringer, Matthew P., and David B. Spencer. "Satellite Constellation Design Tradeoffs Using Multiple-Objective Evolutionary Computation." Journal of Spacecraft and Rockets, 2006: 14041411.

22 Ferringer, Matthew P., and William R. Whittecar. "Global Coverage Constellation Design Exploration Using Evolutionary Algorithms." AIAA/AAS Astrodynamics Specialist Conference. San Diego: AIAA, 2014. 1-20.

23 Ferringer, Matthew P., David B. Spencer, Patrick M. Reed, Ronald S. Clifton, and Timothy G. Thompson. "Pareto-hypervolumes for the Reconfiguration of Satellite Constellations." AIAA, 2008: 18-21.

24 Ferringer, Matthew P., Ronald S. Clifton, and Timothy G. Thompson. "Constellation Design with Parallel Multi-Objective Evolutionary Computation." AIAA, 2006: 21-24.

25 Frayssinhes, E., P. Janniere, and E. Lansard. "The Use of Genetic Algorithms in the Optimization of Satellite Constellations." Spaceflight Dynamics, 1995.

26 Frayssinhes, Eric. "Investigating New Satellite Constellation Geometries with Genetic Algorithms." AIAA, 1996: 582-586.

27 Frayssinhes, Eric, Erick Lansard, and Jean-Luc Palmade. "Global Design of Satellite Constellations: A Multi-Criteria Performance Comparison of Classical Walker Patterns and New Design Patterns." Acta Astronautica, 1998: 555-564.

28 Ghosh, Ashish, and Mrinal K. Das. "Non-dominated Rank based Sorting Genetic Algorithms." Fundamenta Informaticae, 2008: 231-252.

29 Ghosh, Ashish, and Mrinal Kanti Das. Non-dominated Rank based Sorting Genetic Algorithms. Report, Kolkata: IOS Press, 2008. 
30 Hubing, Nick. "Buying Optical Satellite Imagery? The Top 10 Things to Consider." Earth Imaging Journal. 2012. http://eijournal.com/2012/buying-optical-satellite-imagery (accessed 2014).

31 Knowles, J., and D. Corne. "The Pareto Archived Evolution Strategy: A New Baseline Algorithm for Pareto Multiobjective Optimisation." Journal of Evolutionary Computation, 1999.

32 Kollat, J. B., and P. M. Reed. "Comparing State-of-the-Art Evolutionary Multi-Objective Algorithms for Long-Term Groundwater Monitering Design." Advances in Water Resources, 2006: $792-807$.

33 Kollat, Joshua B., and Patrick M. Reed. "The Value of Online Adaptive Search: A Performance Comparison of NSGAII, e-NSGAII, and eMOEA." Evolutionary Multi-Criterion Optimization. Guanajuato: Springer, 2005. 386-398.

34 Lansard, Erick, Eric Frayssinhes, and Jean-Luc Palmade. "Global Design of Satellite Constellations: A Multi-Criteria Performance Comparison of Classical Walker Patterns and New Design Patterns." Acta Astronautica, 1998: 555-564.

35 Mao, Tengyue, Wuhan Hubei, Zhengquan Xu, and Rui Hou. "Efficient Constellation Design Based on Improved Non-dominated Sorting Genetic Algorithm-II." Journal of Computers, 2012: $1337-1344$

36 Mason, William J., Victoria Coverstone-Carroll, and John W. Hartmann. "Optimal Earth Orbiting Satellite Constellations via a Pareto Genetic Algorithm." AIAA, 1998: 169-177.

37 National Geospatial Intelligence Agency. "WGS 84 Earth Gravitational Model." NGA. April 29, 2013. http://earth-info.nga.mil/GandG/wgs84/gravitymod/ (accessed 2014).

38 Pegher, Douglas J., and Jason A. Parish. Optimizaing Coverage and Revisit Time in Sparse Military Satellite Constellations: A Comparison of Traditional Approaches and Genetic Algorithms. Master's Thesis, Monterey: Naval Postgraduate School, 2004.

39 Pohlheim, Hartmut. "GEATbx: Genetic and Evolutionary Algorithm Toolbox for use with MATLAB Documentation." Software Documentation, 2007.

40 Schaffer, J. D. "Multiple Objective Optimization with Vector Evaluated Genetic Algorithms." Proceedings of an International Conference on Genetic Algorithms and their Applications. IEEE, 1985. 93-100.

41 Scialom, U. Optimization of Satellite Constellation Reconfiguration. Master's Thesis, Cambridge: Massachusetts Institute of Technology, 2003.

42 Siddiqi, Afreen, Jason Mellein, and Olivier L. de Weck. "Optimal Reconfigurations for Increasing Capacity of Communication Satellite Constellations." AIAA, 2005: 18-21. 
43 Smith, James Earl. Application of Optimization Techniques to the Design and Maintenance of Satellite Constellations. Master's Thesis, Cambridge: Massachusetts Institute of Technology, 1999.

44 Theoprakt. Orbital Elements. October 10, 2007.

http://en.wikipedia.org/wiki/Orbital_elements\#mediaviewer/File:Orbit1.svg (accessed 2014).

45 Vallado, David A. Fundamentals of Astrodynamics and Applications, 4th ed. Microcosm Press, 2013.

46 Vinti, John P. Orbital and Celestial Mechanics. AIAA, 1998.

47 Vtipil, Sharon D. Constrained Optimal Orbit Design for Earth Observation. PhD Thesis, Norfolk: Old Dominion University, 2010.

48 Wang, Li, Yanjuan Wang, Kewei Chen, and Hui Zhang. "Optimization of Regional Coverage Reconnaissance Satellite Constellation by NSGA-II Algorithm." IEEE, 2008: 20-23.

49 Wertz, James R. Orbit \& Constellation Design \& Management. Hawthorne: Microcosm Press, 2009.

50 Wertz, James R., David F. Everett, and Jeffery J. Puschell. Spce Mission Engineering: The New SMAD. Hawthorne: Microcosm Astronautics Books, 2011.

51 Zitzler, E., and L. Thiele. "Multiobjective Evolutionary Algorithms: A Comparative Case Study and the Strength Pareto Approach." IEEE, 1999: 257-271.

52 Zitzler, Eckart, Marco Laumanns, and Lothar Thiele. SPEA2: Improving the Strength Pareto Evolutionary Algorithm. TIK-Report 103, Zurich: Swiss Federal Institute of Technology, 2001. 